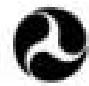

U. S. Department of Transportation

Federat Railroad Administration

\title{
Human Factors Guidelines for Locomotive Cabs
}

Otfice of Pesearch

and Development

Washington. D.C. 20590
U.S. Department of Transportation

John A. Volpe National Transportation Systems Center

55 Broadway

Cambridge MA 02142-1093

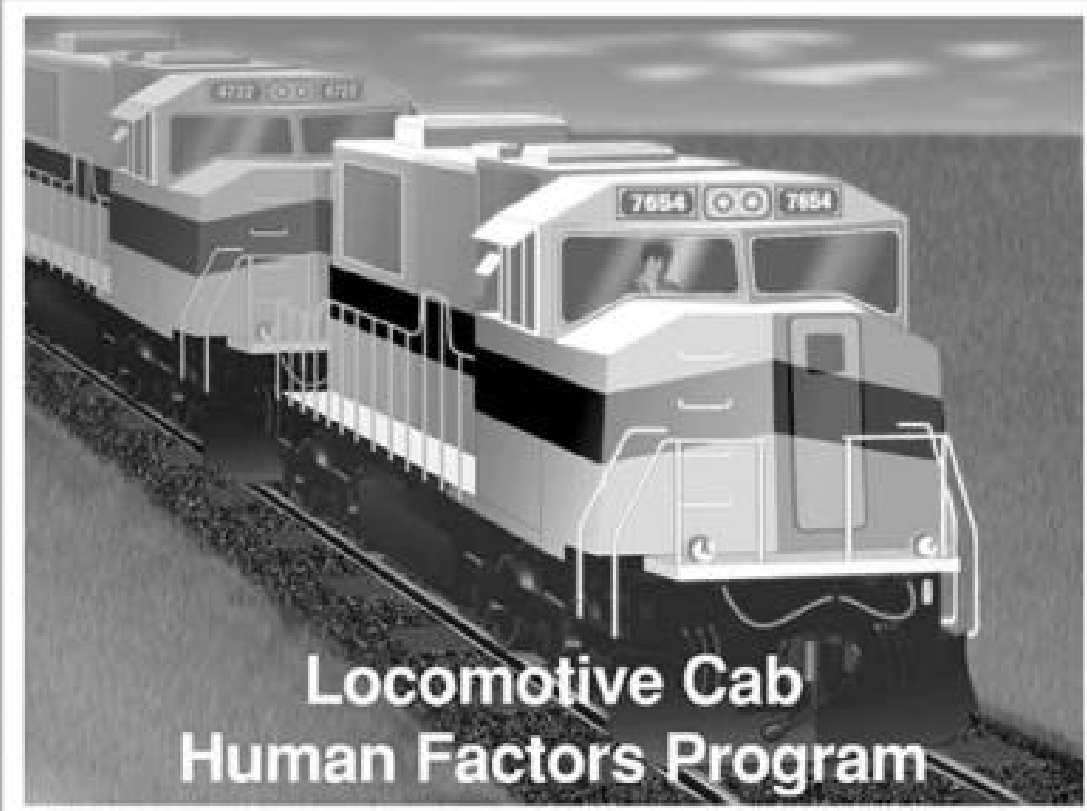

DOT/FRA/ORD-98/03 DOT-VNTSC-FRA-98-8
Final Report

November 1998

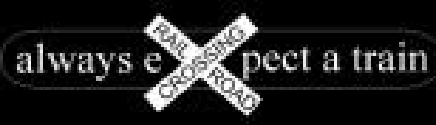

This document is available to the U. S. public through the National Technical information Sarvice, Springfleld, VA 22161 


\section{REPORT DOCUMENTATION PAGE}

Form Approved

OMB No. 0704-0188

Public reporting burden for this collection of information is estimated to average 1 hour per response, including the time for reviewing instructions, searching existing data sources, gathering and maintaining the data needed, and completing and reviewing the collection of information. Send comments regarding this burden estimate or any other aspect of this collection of information, including suggestions for reducing this burden, to Washington Headquarters Services, Directorate for Information Operations and Reports, 1215 Jefferson Davis Highway, Suite 1204, Arlington, VA 22202-4302, and to the Office of Management and Budget, Paperwork Reduction Project (0704-0188), Washington, DC 20503.

\begin{tabular}{|c|c|c|}
\hline 1. AGENCY USE ONLY (Leave blank) & $\begin{array}{l}\text { 2. REPORT DATE } \\
\text { November } 1998\end{array}$ & $\begin{array}{l}\text { 3. REPORT TYPE AND DATES COVERED } \\
\text { Final Report } \\
\text { April } 1994 \text {-May } 1998\end{array}$ \\
\hline
\end{tabular}

\section{TITLE AND SUBTITLE}

Human Factors Guidelines for Locomotive Cabs

6. $\operatorname{AUTHOR}(\mathrm{S})$

Jordan Multer, Robert Rudich, Kevin Yearwood

7. PERFORMING ORGANIZATION NAME(S) AND ADDRESS(ES)

U.S. Department of Transportation

Research and Special Programs Administration

John A. Volpe National Transportation Systems Center

5. FUNDING NUMBERS

55 Broadway

Cambridge, MA 02142-1093

9. SPONSORING/MONITORING AGENCY NAME(S) AND ADDRESS(ES)

U.S. Department of Transportation

Federal Railroad Administration

Office of Research and Development

RR928/R9228

4007 th Street, S.W.

Washington, DC 20590

\section{SUPPLEMENTARY NOTES}

12a. DISTRIBUTION/AVAILABILITY STATEMENT

12b. DISTRIBUTION CODE

This document is available to the public through the National Technical Information Service, Springfield, VA 22161

\section{ABSTRACT (Maximum 200 words)}

This document presents human factors guidelines for the evaluation of the locomotive cab. These guidelines are part of an effort to evaluate working conditions and safety in the locomotive cab. The guidelines will serve as a decision-making tool for evaluating current and proposed locomotive designs and in particular Association of American Railroads (AAR) standards for defining industry requirements in cab design.

The human factors concerns addressed by the guidelines can be divided into two themes: working conditions and information technology. Chapters within the guidelines address the following topics: heating, ventilation, air conditioning, noise, vibration, toilet facilities, general considerations for cab layout, ingress and egress, visibility, seating and workstation design. Workstation design includes both hardware and software issues. Within each chapter, human factors considerations are presented within the context of relevant operational issues and specific recommendations are offered.

The guidelines were developed from a variety of sources. Journal articles, handbooks, reference guides and papers applicable to human-machine systems in general as well as literature specific to the problems of locomotive cab design and operation were examined. To make the guidelines as relevant as possible, members of the railroad community (major railroads, manufacturers, AAR, the railroad unions) were consulted.

\section{SUBJECT TERMS}

human factors, locomotive cab, design guidelines, locomotive design, working conditions, safety

\section{SECURITY}

CLASSIFICATION OF REPORT

Unclassified

\section{SECURITY \\ CLASSIFICATION \\ OF THIS PAGE}

Unclassified
19. SECURITY

CLASSIFICATION

OF ABSTRACT

Unclassified
15. NUMBER OF PAGES 230

16. PRICE CODE 


\section{PREFACE}

In 1992, Congress passed legislation (PL 102-365), requiring the Federal Railroad Administration (FRA) to complete rulemaking activities relating to the improvement of safety and working conditions in locomotive cabs. The FRA is charged with the task of assessing standards developed by the Association of American Railroads (AAR) on crashworthiness and working conditions that affect safety and productivity. As part of this effort, the FRA conducted research to address these issues.

The development of human factors guidelines for the locomotive cab is part of the FRA's research effort to evaluate working conditions and safety in the locomotive cab. The guidelines will serve as a decision-making tool for evaluating current and proposed locomotive designs and, in particular, standards developed by the AAR for defining basic industry requirements in cab design.

Human factors guidelines can aid in the design and evaluation of new or existing locomotive cabs. While there is a rich body of human factors research, for the most part it was not written and organized for use by those developing or evaluating complex systems, like the locomotive cab. The available literature is located in diverse places that include journal articles, technical publications, textbooks and conference proceedings. Where there are human factors guidelines available, they are often general in nature and have not been tailored to accommodate the specific requirements of the railroad environment.

This document represents the beginning of a process. Our understanding of human factors issues affecting locomotive engineers and others who work in the cab is still incomplete. As this document is being prepared, many of the issues surrounding the incorporation of new information technology are still being debated. The currently available literature frequently does not address the capabilities of this new technology (Rogers and Myers, 1993). Future research and continuing dialogue will be needed as the locomotive cab evolves to incorporate this new technology. How the locomotive engineer will interact with this new technology should be a central part of this debate.

To develop the guidelines, the authors collected and reviewed papers, handbooks, reference guides applicable to human-machine systems in general as well as materials specific to the problems of locomotive cab design and operation. To make the guidelines as relevant as possible, members of the railroad community were consulted (the major railroads, manufacturers, AAR and the railroad unions) to better understand the current problems with current cab designs and obtain a sense of what the future needs are likely to be.

In presenting guidelines for evaluating the design of locomotive cabs, current human factors concerns can be divided into two broad themes: working conditions and the incorporation of information technology. Environmental working conditions (e.g., noise and seating) and how it affects operator performance have been a concern of the railroads, manufacturers and train engineers for many years. The second theme revolves around the incorporation of information technology into the locomotive cab. The problems of how to incorporate new information technology, in particular, advanced command, control and communication systems, is a more recent concern and is growing in importance. 
Most of this report focuses on issues related to working conditions. This emphasis reflects the concerns of engineers who have identified many of the ergonomic problems within the locomotive cab. Currently, those concerns center on crew comfort. As those problems are solved, the relative effort focusing on information processing as it relates to train control will grow.

\section{Working conditions}

In the past, locomotive cab designs evolved without the benefit of human factors support (Gamst, 1975). Crew comfort in the cab compartment trailed that found in passenger compartments and other working environments. Noise, ventilation, and seating are three examples of problem areas that affected crew comfort, safety, and productivity. Although human factors considerations are an important consideration in the design of the current generation of locomotives, the long service life of locomotives means that these concerns will exist for some time. Approximately, 19 percent of locomotives in service in 1994 were built before 1970 (AAR, 1994). Thus, noise levels in a significant number of cabs exceed federal regulations for this working environment and are loud enough to permanently impair hearing (Gamst, 1975). Railroads have responded to this problem by providing hearing protection equipment. The lack of proper ventilation in the cab results in hot and cold spots in cab, inadequate heating in cold weather, and inadequate cooling in hot weather as well as noxious fumes vented inside the cab (Gamst, 1975). These conditions contribute to health problems and crew fatigue.

In terms of physical accommodations, the lack of appropriate seating is one of most thoroughly documented problems in the cab environment and can contribute to fatigue and injury. Other important human factors concerns with working conditions in the cab include: safely entering and exiting the cab, adequate visibility, and sanitary toilet facilities.

\section{Incorporating information technology}

As we approach the twenty-first century, manufacturers and railroad operators are introducing new information technology into the locomotive cab in an attempt to improve train control. The incorporation of this technology will affect how the locomotive engineer performs his or her job.

In this document, concerns for incorporating the new information technology into the locomotive revolve around the interface with which the locomotive engineer will interact with to receive information and control train movements. In the past, this interface consisted of electromechanical dials, gauges and levers, and control knobs. This interface is being replaced with workstations that consist of computer displays and controls (i.e., CRT or LCD displays and keyboards). New technology and new designs may not necessarily make new locomotives better than previous versions. The design of a complex man-machine system, like a locomotive cab, involves making trade-offs and compromises to meet customer requirements and yet stay within cost and schedule limitations. This knowledge will be acquired over time as locomotive cab designs evolve and we gain experience from other transportation modalities, as well as testing the usability of proposed designs.

\section{Operational Issues}


The design of the locomotive cab has been influenced by a variety of operational issues. These issues represent the needs of the engineers, conductors and maintenance staff who will work in this environment as well as the constraints faced by the manufacturers who design and build the locomotive and the railroads who purchase and operate them. Some of the major operational issues are discussed below:

\section{- Incremental pace of change}

Successive generations of locomotive cab designs evolved in incremental steps. Some characteristics date back to steam era traditions. The long service life of locomotives makes compatibility with previous designs an important issue, since a railroad may own locomotives with cab configurations from different design eras.

\section{- Backward compatibility}

The desire for backward compatibility by which locomotive cabs evolved has both positive and negative human factors implications. The incorporation of new features which address new capability while retaining old features helps the engineer to learn the new system more quickly, since only the new features must be learned. Where the control-display relationships remain the same, the engineer may avoid committing errors due to negative transfer. However, backward compatibility has also resulted in the interface, itself, limiting the kinds of changes that take place, rather than the user requirements.

\section{- Interaction between railroad and locomotive manufacturer}

Interaction between the railroads and manufacturers is the major influence in the design process. Standardization makes design and manufacturing easier for the builder and operation easier for the end user, the engineer (when transitioning from one cab to another). However, railroad properties can specify considerable individual modifications. Ergonomic considerations, including consideration of the anthropometry of the user population, are often out of the hands of the builder. It must put together the components to give the customers what they want, even where customer requirements deviate from the ideal. For some areas (e.g., noise limits), there are currently accepted standards that are based on practical limitations. Where these standards exist, they are cited. However, the report also cites goals which should be strived for and that are more supportive of the safety, health productivity and comfort of the engineer.

\section{- Bi-directional operations}

A key issue that will affect future locomotive cab designs is the need for bi-directional operations. A number of proposed designs are being considered to address bi-directionality in future locomotive cabs. One proposal would place the controls in the arm rest of the engineers' chair and place duplicate sets of displays facing in both directions. Another proposal would divide the locomotive into two units, one containing the cab controls and another generating the power. 
The locomotive cab would be isolated from the power unit and could be turned around to operate in either direction.

\section{- Maintenance}

Maintenance costs associated with normal maintenance as well as with the repair or replacement of failed parts may exceed the initial cost of the locomotive over its lifetime. A hidden cost is the effect on performance, safety, and working conditions when various subsystems and components within the cab are not adequately maintained.

\section{Organization of the Report}

The guidelines are organized into three chapters: Environment, Layout, and Workstation.

- The environment chapter addresses issues like heating, ventilation, air conditioning, vibration, and noise that affect the overall working conditions in which the engineers and conductors operate.

- The layout chapter addresses issues like general considerations, ingress and egress, visibility, and seating that define the engineer's physical relationship with the equipment in the cab and the outside environment.

- The workstation design chapter addresses topics like controls, displays, auditory devices, and computer input. These topics address how the engineer obtains information about the status of the locomotive and how train control is implemented.

Each chapter provides two basic elements: human factors considerations and operational issues. The discussion of operational issues is specific to the locomotive cab environment. The human factors considerations provide a background discussion of the research contributing to individual guidelines. Chapter 1 gives an overview of the issues that are influencing future locomotive cab designs and basic human factors principles that need to be considered while chapter 2 contains key points from each of the following chapters. The key points summarize the specific human factors recommendations for a particular issue. 
In this report measurements are presented in English units except where the convention is to display this information in metric units. U.S. railroads have usually used English units (i.e., feet and inches) to indicate measurement quantities. Recently, locomotive manufacturers have adopted the use of metric units for indicating measurement information in their designs.

Measurement information derived from standards developed by the International Organization for Standardization (ISO) also use metric units. Conversion factors are presented the beginning of this document to aid the reader to convert between metric or English units. 


\section{ACKNOWLEDGEMENTS}

In 1992, Congress passed legislation (PL 102-365), requiring the Federal Railroad Administration (FRA) to complete rule making activities relating to the improvement of safety and working conditions in locomotive cabs. The FRA is charged with the task of assessing standards developed by the Association of American Railroads (AAR) on crashworthiness and working conditions that affect safety and productivity. As part of this effort, the FRA must conduct research and analysis to address these issues.

The development of human factors guidelines for the evaluation of the locomotive cab is part of the FRA's research effort to evaluate working conditions and safety in the locomotive cab. The guidelines will serve as a decision-making tool for evaluating current and proposed locomotive designs and in particular, standards developed by the AAR for defining basic industry requirements in cab design.

To accomplish the task of developing guidelines, we performed a review of the literature and spoke with representatives of the major railroads, manufacturers, AAR and the railroad unions. Authors collected and reviewed papers, handbooks, reference guides applicable to humanmachine systems in general as well as materials specific to the problems of locomotive cab design and operation. To make the guidelines as relevant as possible we spoke with members of the railroad community to better understand the current problems with current cab designs and obtain a sense of what the future needs are likely to be and how they may be accommodated.

This study was funded through the Federal Railroad Administration's Office of Research and Development and managed by Garold Thomas. We would like to thank Garold Thomas, Steve Ditmeyer, and Tom Raslear for their valuable comments and feedback as this report evolved.

We would also like to thank the many individuals who contributed to this document. We owe a special thanks to Tim Jones of AAR, who gave us a broad picture of the current issues with which the railroad industry is wrestling. We are grateful to the members of the EMD design group, John Handley, Harvey Boyd, Jay Davis, Rick Davis, Terry McCarl, John Stringer, Bill Donley, Walter Cleric and Marty Steel for sharing with us the manufacturer's design concerns and providing comments on a draft. We would like to thank Francois Laporte of CN, Rick Schultz of GE, and George Keuhn of IITRI for their thoughtful comments. As part of the review process, Michael Coplen and Donald Sussman offered many excellent suggestions that immeasurably improved the quality of this document. Finally, we would like to thank Anita Graffeo of EG\&G Services for her editorial expertise and help in putting this document together. 


\section{TABLE OF CONTENTS}

Chapter

$\underline{\text { Page }}$

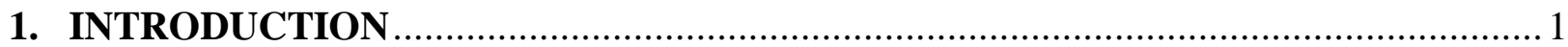

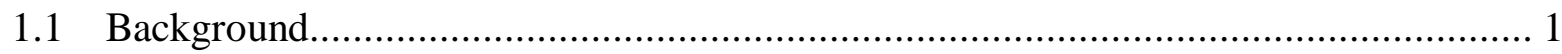

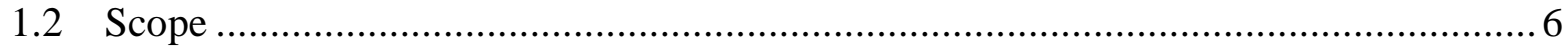

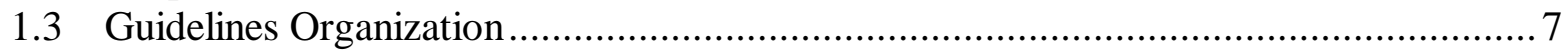

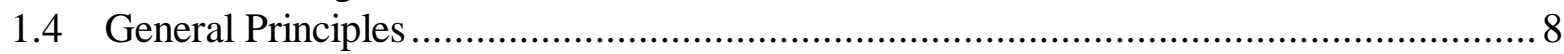

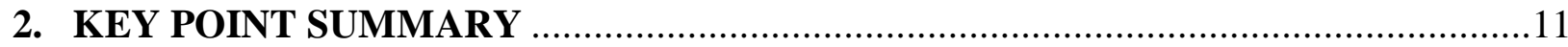

2.1 CAB ENVIRONMENT ............................................................................ 11

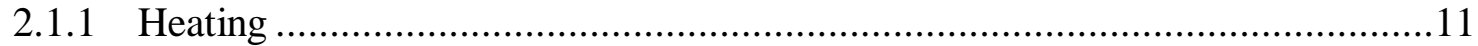

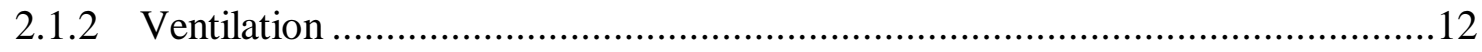

2.1.3 Air Conditioning ............................................................................ 12

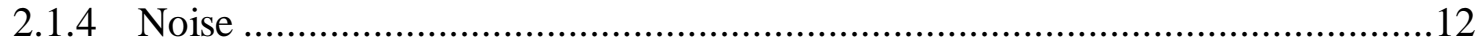

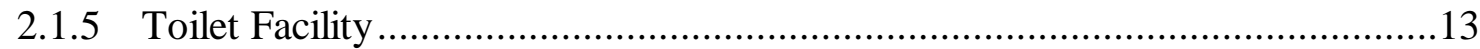

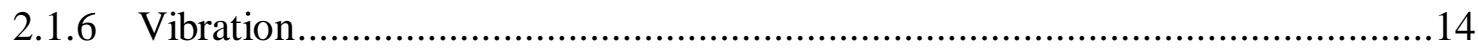

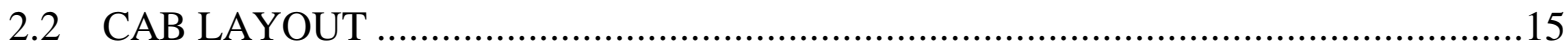

2.2.1 General Design............................................................................ 15

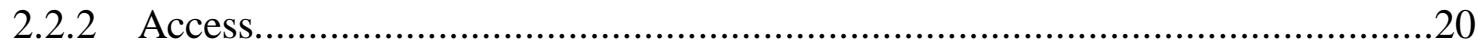

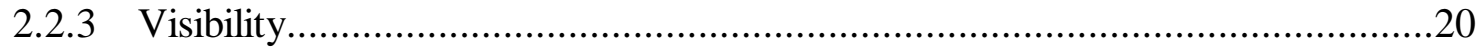

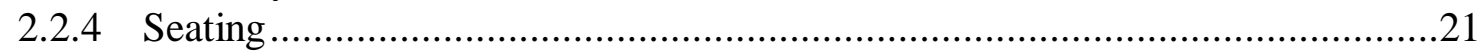

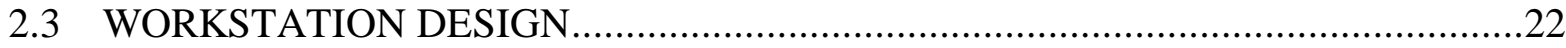

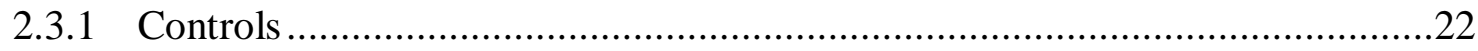

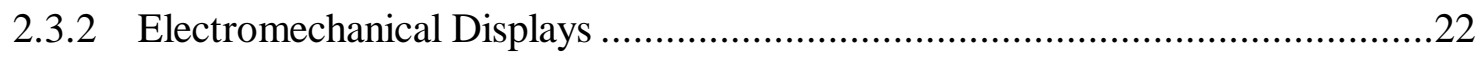

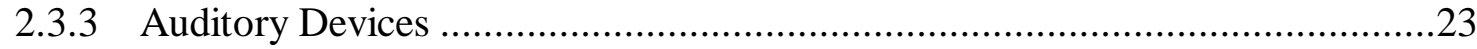

2.3.4 General Principles ...............................................................................24

2.3.5 Automation .......................................................................................... 28

2.3.6 Electronic (Computer Generated) Displays ...........................................29

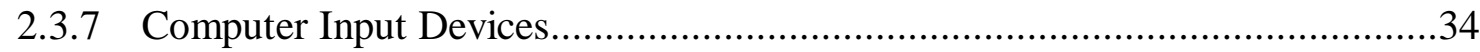

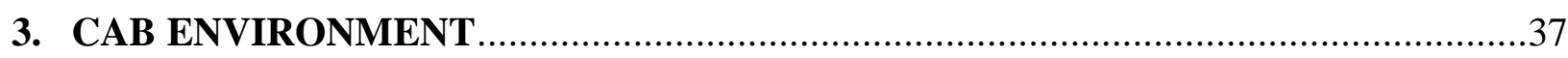

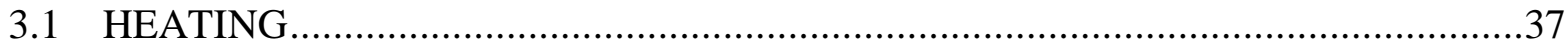

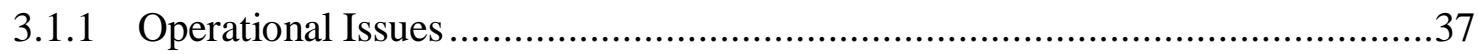

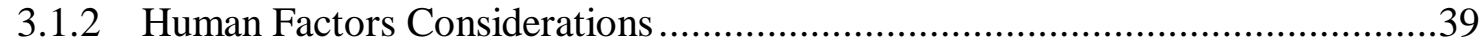

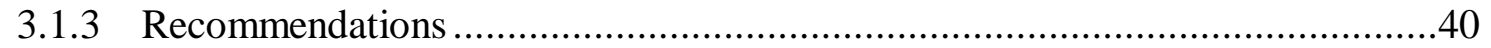




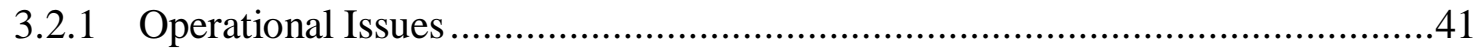

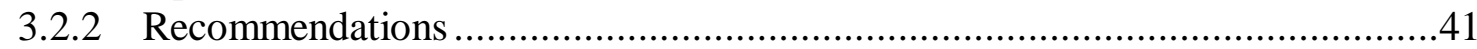

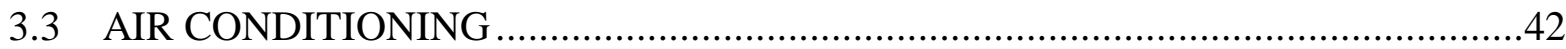

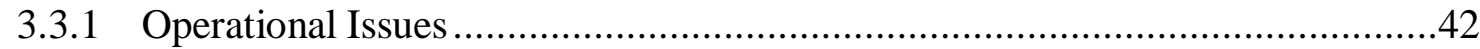

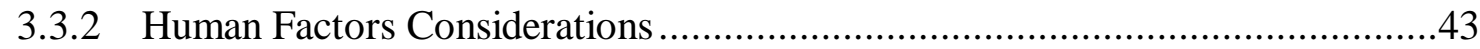

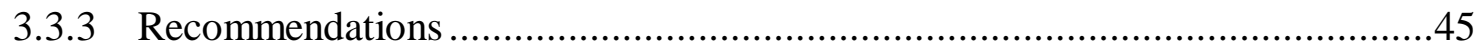

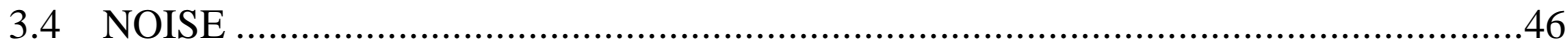

3.4.1 Operational Issues ..........................................................................46

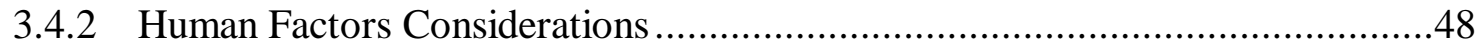

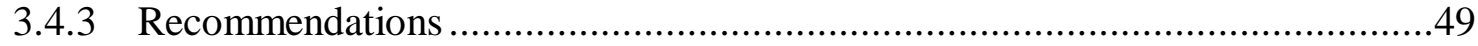

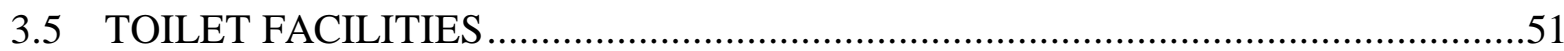

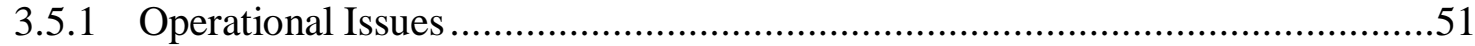

3.5.2 Human Factors Considerations ..............................................................53

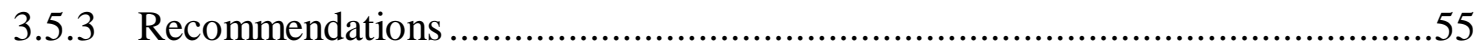

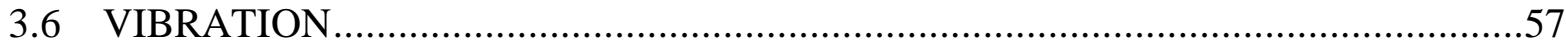

3.6.1 Operational Issues ...........................................................................57

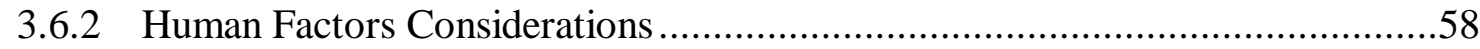

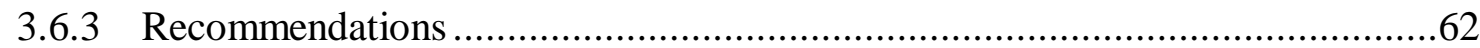

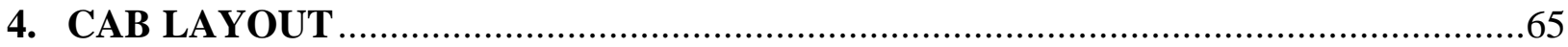

4.1 GENERAL DESIGN CONSIDERATIONS …...........................................65

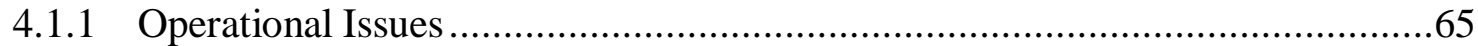

4.1.2 Human Factors Considerations .............................................................68

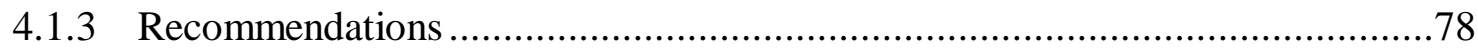

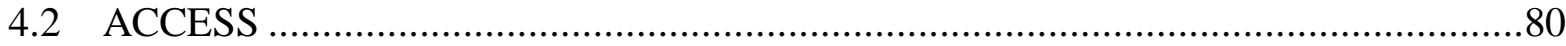

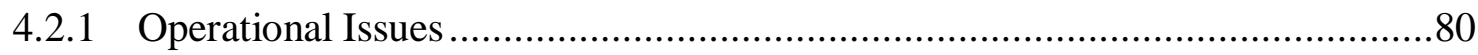

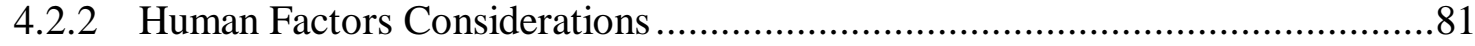

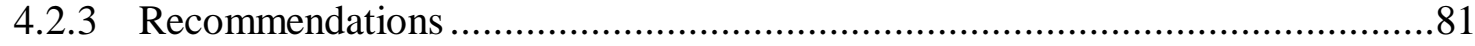

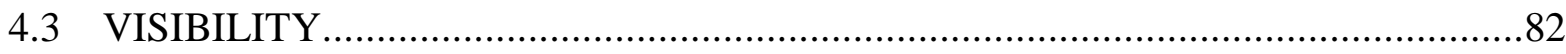

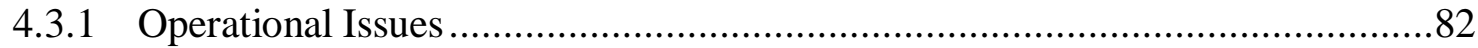

4.3.2 Human Factors Considerations ............................................................83 


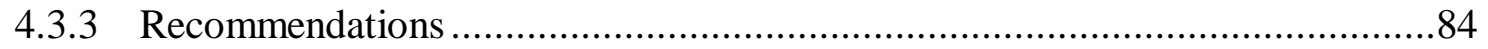

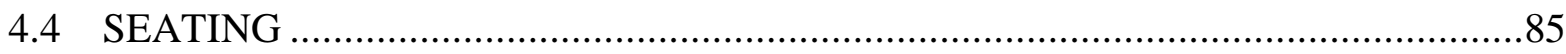

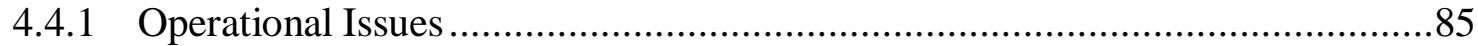

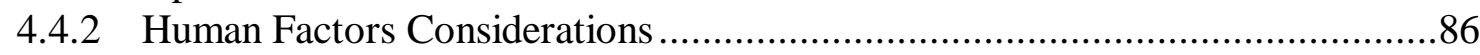

4.4.3 Recommendations ........................................................................ 90

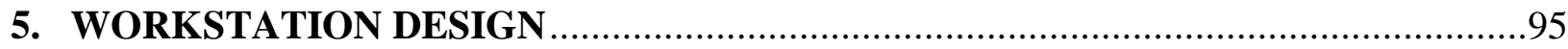

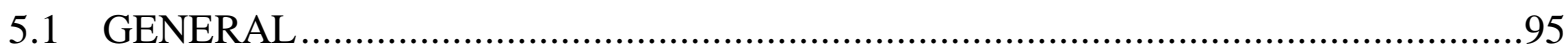

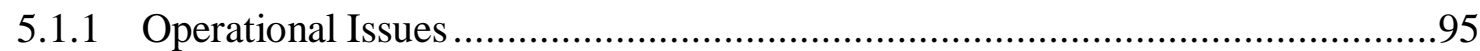

5.1.2 Human Factors Considerations ............................................................. 98

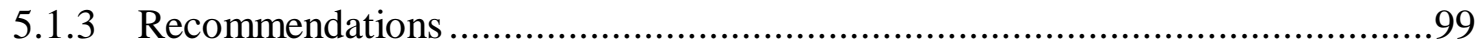

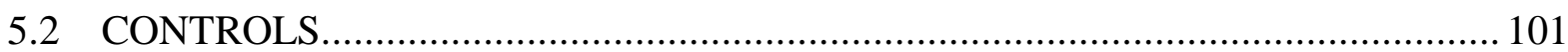

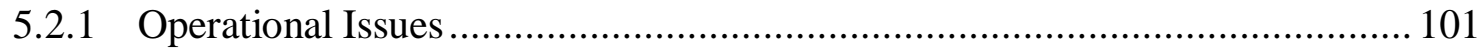

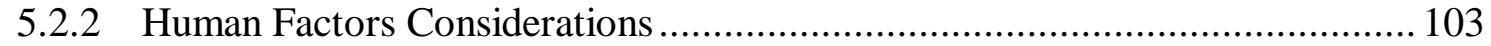

5.2.3 Recommendations ......................................................................... 104

5.3 ELECTROMECHANICAL DISPLAYS ....................................................... 106

5.3.1 Operational Issues ....................................................................... 106

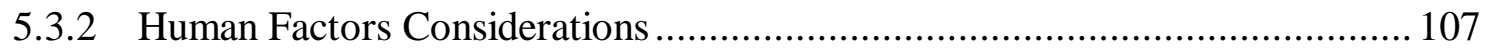

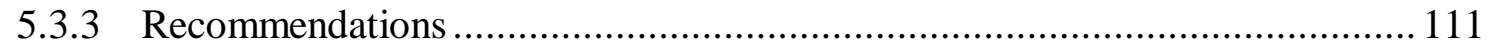

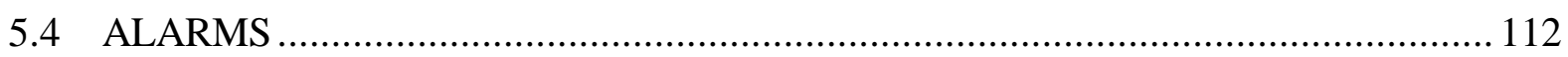

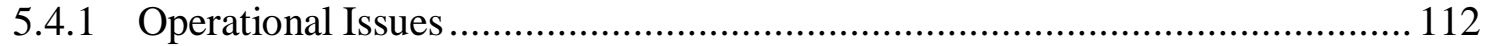

5.4.2 Human Factors Considerations ............................................................. 114

5.4.3 Recommendations ...................................................................... 114

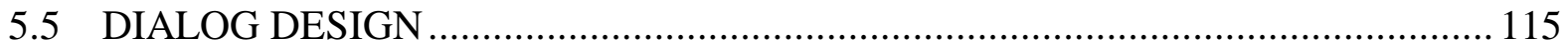

5.5.1 Operational Issues ........................................................................... 116

5.5.2 Human Factors Considerations .............................................................. 117

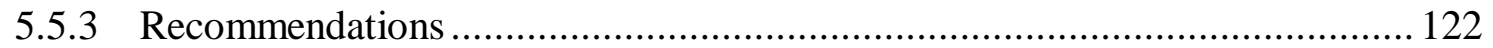

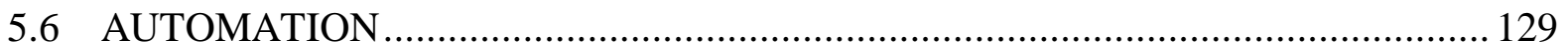

5.6.1 Operational Issues .......................................................................... 129

5.6.2 Human Factors Considerations ........................................................ 131

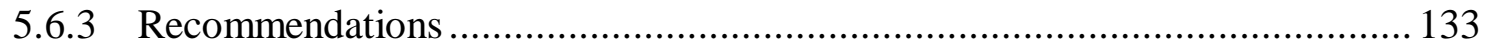


5.7 ELECTRONIC (COMPUTER GENERATED) DISPLAYS .

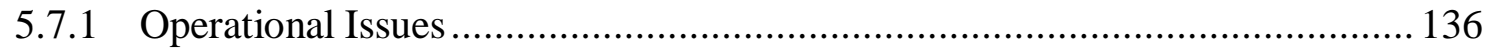

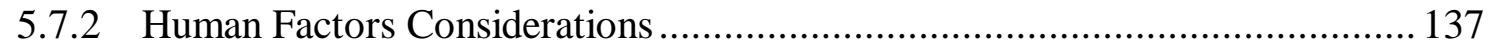

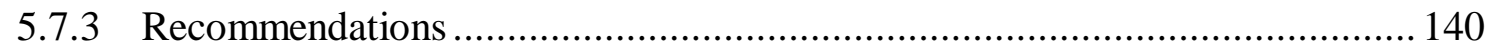

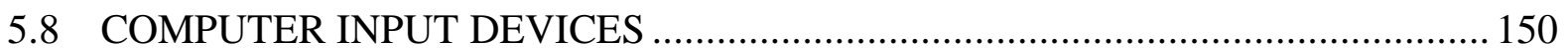

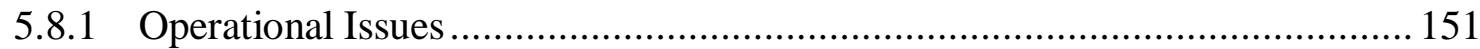

5.8.2 Human Factors Considerations ……………............................................... 151

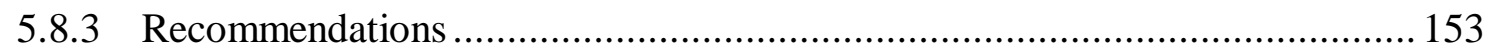

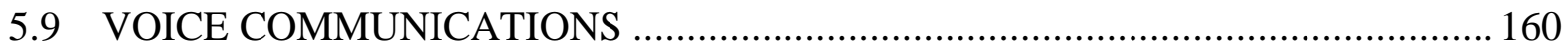

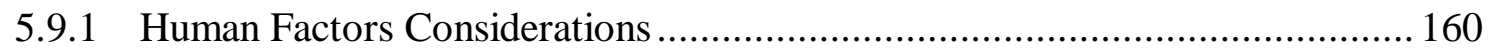

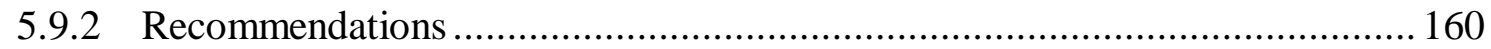

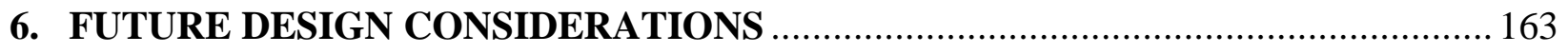

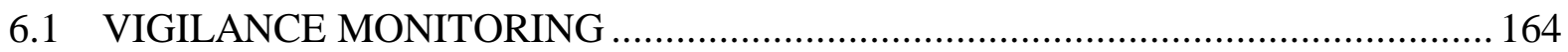

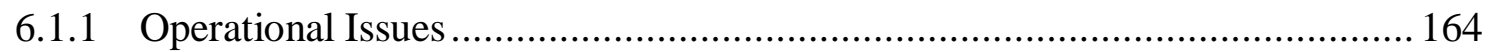

6.1.2 Human Factors Considerations ………………….................................. 165

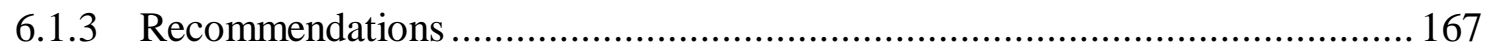

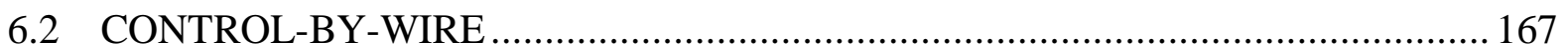

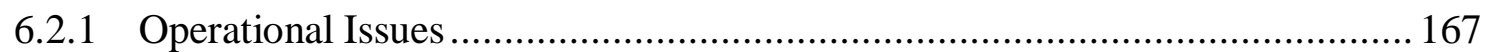

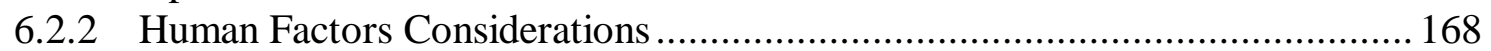

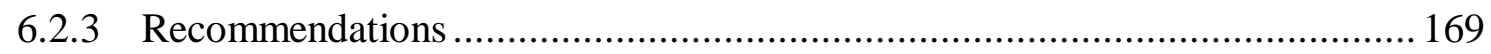

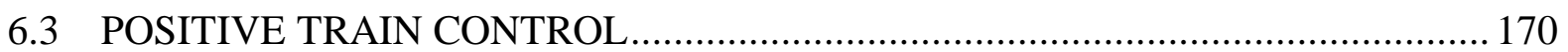

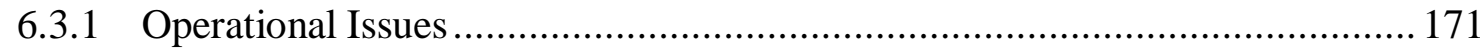

6.3.2 Human Factors Considerations ............................................................... 174

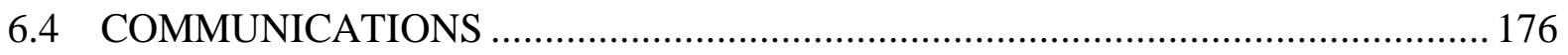

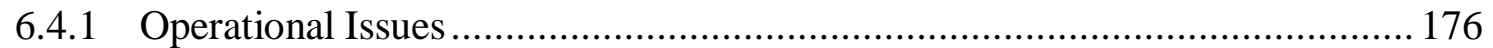

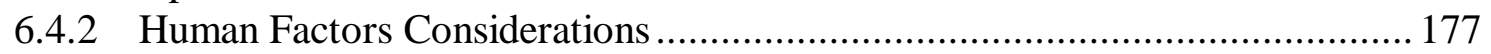

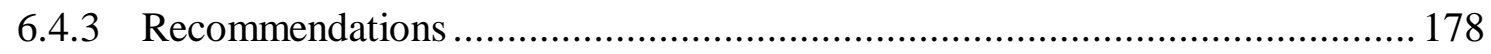

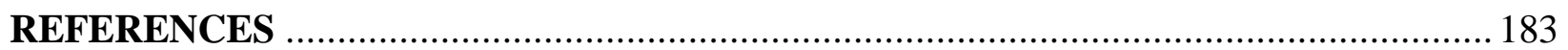

INDEX … 


\section{LIST OF FIGURES}

\section{$\underline{\text { Figure }}$}

$\underline{\text { Page }}$

3-1 Work Environment Comfort Zones ................................................................... 38

3-2 Relationship between Temperature and

Unsafe Behaviors

3-3 ASHRAE Standard for Thermal Comfort ........................................................45

3-4 Vent Locations of Locomotive Lavatory...............................................................52

3-5 Vibration Weighting Values ....................................................................61

4-1 Raised Platform for Engineer's Seat .................................................................... 70

4-2 Area within which Primary Displays Should be Located ............................................72

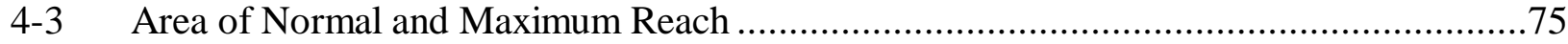

4-4 Recommended Heights for Four Classes of Controls ...........................................75

4-5 Locomotive Engineer Vision Requirements.......................................................83

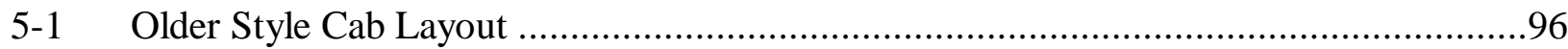

5-2 Modern Style Cab Layout .......................................................................... 97

5-3 Top View - Zones of Comfort and Reach..................................................... 100

5-4 Front View - Zones of Comfort and Reach ..................................................... 101

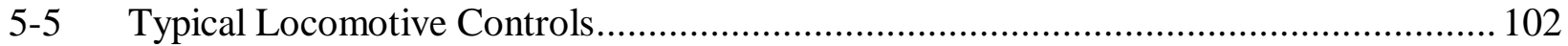

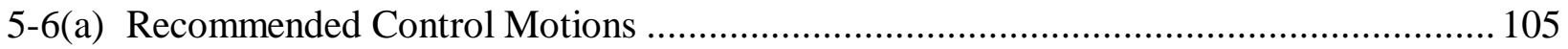

5-6(b) Recommended Control Motions for

5-7 Recommended Scale Mark Dimensions.......................................................... 109

5-8 Recommended Placement of Visual Alert Signals .................................................. 110

5-9 Visibility of Controls and Displays for a

Seated Locomotive Engineer 


\section{LIST OF FIGURES (cont'd.)}

$\underline{\text { Figure }}$

$\underline{\text { Page }}$

5-10 Poor and Improved Function Key Design.................................................... 123

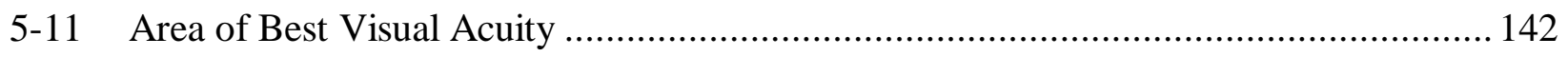

5-12 Comparison of Typefaces........................................................................... 146

5-13 Example of Three Electronically Generated

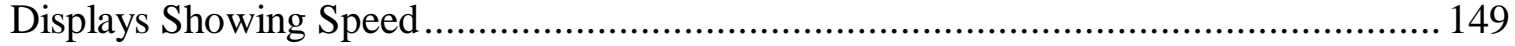

5-14 Keyboard Dimensions, Resistance, and Displacement ...................................... 156

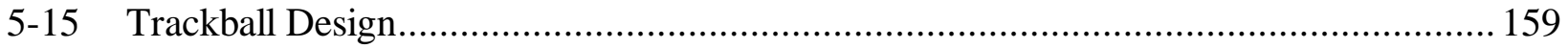

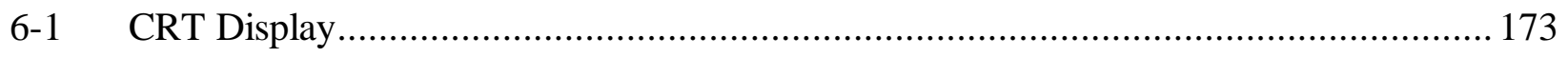




\section{LIST OF TABLES}

$\underline{\text { Table }}$

$\underline{\text { Page }}$

5-1 Minimum Diameter of Inner Annulus at

Various Viewing Distance

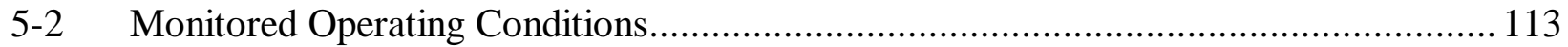

5-3 Advantages and Disadvantages from Four

Interactions Styles

5-4 Suggested Response Times by Task

5-5 One Set of Recommended Heights of Alphanumeric

Characters at 28 inch Viewing Distance 146

6-1 Possible Vigilance Device States 165 


\section{CHAPTER 1. INTRODUCTION}

\subsection{BACKGROUND}

As the locomotive evolved from the steam engine to the dieselelectric engine, new technology has also been added. This new technology has affected how the train operators perform their jobs. The number of people in the cab has also changed along with the functions they perform. The cab space was occupied by the engineer, conductor, brakeman and fireman. Today, the fireman and brakeman are gone. The cab crew usually consists of an engineer and conductor. During the days of steam, the cab was a male environment, but today, women are entering careers as railroad conductors and train engineers.

New communications and signaling systems, digital data links, Global Positioning System (GPS), and innovative computer technology will make it feasible for dispatchers in central control centers to monitor the condition of the locomotive and send train orders directly to the locomotive cab. Successful implementation of new technology in the cab will require careful consideration of the activities performed in the cab and how the engineer relates to the rest of the system. Effective design of the entire locomotive cab is one part of the equation. Evaluating these new designs will require understanding the operator's capabilities and limitations in performing his or her duties.

Current human factors guidelines for the evaluation of the design of locomotive cabs can be divided into two broad themes: working conditions and the incorporation of information technology. Working conditions (e.g., noise and seating) and their effects on operator performance have been a concern of the railroads, manufacturers, and train engineers for many years. The second theme, the incorporation of information technology into the locomotive cab, is of growing importance.

The bulk of this report focuses on issues related to working conditions. This emphasis reflects the concerns of locomotive engineers who have identified many of the ergonomic problems within the locomotive cab. Currently, those concerns center on crew comfort and safety. As those problems are solved, the relative effort that focuses on information processing as it relates to train control will grow. 


\section{Working conditions}

Current locomotive designs evolved from steam-driven locomotives dating from decades ago. Conditions under which the locomotive engineer operated were radically different from those of today. In early steam-driven locomotives, physical train control involved a train engineer, fireman and brakeman. For example, depression of the deadman footplate required the force of two people, the engineer and the fireman (Ford, 1978). The fireman also shared responsibility with the train engineer for power generation and monitoring the visual scene (Branton, 1978). With the advent of the diesel-electric locomotive, the engineer became the sole agent of the physical aspects of train control.

The early steam-driven locomotives provided a harsh work environment in which the operators were exposed to extremes of temperature, noise, and noxious fumes. Although the cab environment improved as diesel-electric locomotives replaced steam-powered locomotives, the locomotive cab is still harsher than the typical office environment. Working conditions are important because they affect the safety and productivity of the operator as well as comfort.

In the past, locomotive cab designs evolved without the benefit of human factors support (Gamst, 1975). Crew comfort in the cab compartment has gradually improved, but trails the passenger compartments. Although human factors considerations are important in the design of the current generation of locomotives, the long service life of locomotives means that change will come slowly. Approximately 19 percent of locomotive in service in 1994 were built before 1970 (AAR, 1994).

The long service life of locomotives means that maintenance will also be a significant issue. Maintenance costs associated with normal servicing, as well as repairing and replacing failed parts, may exceed the initial cost of the locomotive over its lifetime.

Noise, ventilation, and seating are three examples of problem areas that affect crew comfort, safety and productivity. Noise levels in many cabs are loud enough to permanently impair hearing (Gamst, 1975). Additionally, high levels of noise coupled with hearing losses of many middle-aged and older engineers makes clear communications more difficult, and can impair the operator's ability to detect audible warning signals. The lack of proper ventilation in the cab results in hot and cold 
spots in cab, inadequate heating in cold weather and inadequate cooling in hot weather, as well as noxious fumes vented inside the cab (Gamst, 1975). These conditions may contribute to health problems and crew fatigue. The lack of appropriate seating in the cab environment is one of most thoroughly documented problems. Engineers complain of lower back, neck and shoulder pains related to sitting posture (Hedberg, 1987; Kerst, 1991). The lack of adequate seating results from a mismatch between the anthropometric requirements of train engineers for the seats and control stand and the actual dimensions of this equipment (Kerst, 1991).

Other important human factors concerns with working conditions in the cab include: safely entering and exiting the cab, adequate visibility of the track, signaling system and scenery, and sanitary toilet facilities.

\section{Incorporating information technology}

As we approach the twenty-first century, manufacturers and railroad operators are introducing new information technology into the locomotive cab in an attempt to improve train control. To remain competitive, railroads try to cut costs wherever possible, while responding to the needs of their customers. Shippers are demanding better ways of tracking their goods and insuring that they arrive intact and within the timetables agreed to. As in aviation, the railroads seek methods to maximize the throughput of their system. To accomplish these objectives, the railroads are evaluating information that will result in improved train control. The incorporation of this technology will affect how the locomotive engineer performs his or her job.

One of the major trends in railroad operations affecting the design of new and retrofitted locomotives is the parallel incorporation of computer technology, reductions in the number of operating personnel and increasing automation, in which the equipment performs tasks previously performed by the engineer (Welty, 1994, Progressive Railroading May 1994). Advanced Train Controls Systems (ATCS), Advanced Railroad Electronic System (ARES), Positive Train Control (PTC), Positive Train Separation (PTS), wayside detection systems, and digital radio (i.e., Data Link) represent a few of the concepts and technologies that may find their way into future locomotive cabs.

Before these technologies can be successfully incorporated into 
the locomotive cab of the future, a variety of human factors issues need to be resolved. ATCS, ARES, PTC and PTS have the potential to automate tasks that are currently being done manually. Which tasks will the engineer perform and which will be done by the "system?" The general answer is to try to assign to the "machine" those tasks to which the engineer is least suited and where possible give the engineer those tasks to which he or she is best suited. This will require identifying the tasks to be performed and measuring the engineer's ability to carry out those tasks.

The outcome of this process will affect safety, productivity, and job satisfaction. For example, how will the engineer perform in an emergency if the equipment responsible for effecting changes in train movement authority fails? As in the aviation environment, an autopilot system can lull the operator into a sense of complacency. This situation can lead to one in which the train engineer increasingly serves as a monitor of tasks performed by the "system." In a "safe" system, there will be few failures requiring intervention which can produce boredom and complacency in the crew. However, human operators are poor monitors and, as such, can lead to a lack of situation awareness at a time when a quick response is required. Thus, it is essential that the train engineer be actively involved in train control.

In this document, the concerns for incorporating the new information technology into the locomotive revolve around the interface that the locomotive engineer will interact with to receive information and control train movements. In the past, this interface consisted of electromechanical dials, gauges and levers, and control knobs found in conventional control stands. This interface is being replaced with workstations that consist of computer displays and controls (i.e., CRT displays and keyboards).

However, in making dramatic changes in the interface which train engineers have been using for decades, a word of caution is needed. Although a design standard may meet or exceed specifications established by human factors guidelines, this does not insure acceptance by users. For example, some engineers who operated older locomotives with the control stand for many years find locomotives with the workstation console design uncomfortable. The following quote (Brown, 1994) illustrates the problem:

The P32's cab is very quiet, with good heaters and 
visibility, but unfortunately it has the new desk-style control stand, designed by people who sit at desks! Apparently they have determined that railroaders want to sit at desks, too! You're stuck sitting in one position with this stand, whereas with the old AAR controls, there are five or six positions. This is handy when you're there for 9 to 12 hours.

This opinion suggests that the designer did not take user needs into account. To maximize user acceptance, it is important to involve users during the design and development process beginning at the earliest possible stage when the system requirements are developed.

The quotation above also illustrates another important problem inherent in the design process. Adding new technology and changing the design may not necessarily make new locomotives better than previous versions that lacked the new technology and the new design. The design of a complex man-machine system, like a locomotive cab, often involves making trade-offs and compromises to meet customer requirements and staying within cost and schedule limitations

Human factors guidelines can aid in the design and evaluation of new or existing locomotive cabs. While there is a rich body of human factors research, much of it was not written and organized for use by those developing or evaluating complex systems. The available literature is located in diverse places that include journal articles, technical publications, textbooks, and conference proceedings. Human factors guidelines are available, but they are often general in nature and have not been tailored to accommodate the specific requirements of the railroad environment.

These human factors guidelines for evaluating the locomotive cab contain information applicable to systems in general as well as information specific to the railroad environment.

To promote safety and productivity, user-centered designs are needed to insure that the locomotive engineer can accomplish the tasks that are a part of his or her job. User-centered design has the potential to reduce costs while increasing safety and productivity.

\subsection{SCOPE}


The purpose of this document is to provide human factors guidelines for the design of existing locomotive cabs and proposed designs for use in new or retrofitted locomotives.

The compilation of human factors guidelines provided in this document represents the beginning of a process. Our understanding of human factors issues affecting locomotive engineers is still incomplete. As this document is being prepared, many of the issues surrounding the incorporation of new information technology are still being debated. Human factors guidance is helpful in addressing these issues. However, currently available literature frequently does not specifically address the capabilities of this new technology (Rogers and Myers, 1993). Additional research will be needed to address these issues. A continuing dialogue will be needed as the locomotive cab continues to evolve.

The product of human factors research takes several forms including design principles, design guidelines, and design rules (Helander, 1994; Dumas, 1994). Design principles represent broad recommendations based upon research about how people behave. For example, "Provide feedback to the user about the state of the system." The design principles underlying the guidelines found in this document are presented in Section 2.

Principles, as Dumas indicates, are only goals. Guidelines are general recommendations that are tailored to the environment in which they will be used. Guidelines are more specific than goals. Here is an example of a guideline from Smith and Mosier (1984): "Ensure the computer acknowledges every control entry immediately; for every action by the user there should be some apparent reaction from the computer." The guideline may also include examples and background information describing the research on which the guideline was derived.

Design rules are a series of design specifications for a particular system and are sufficiently detailed that they do not require additional interpretation (Helander, 1994). A design rule might specify the exact message displayed to the operator in response to a request and indicate how quickly to display the message after the request is made. Design rules are necessary because different designers can implement the same guideline in different ways. Design rules enable designers to maximize consistency in the application of design guidelines.

A potential problem with guidelines is that different guidelines 
may offer conflicting advice regarding the implementation of a particular software feature or piece of equipment. The relative importance of a given guideline will depend upon the context in which it is applied. Therefore, trade-offs between conflicting guidelines must be made during the design process. The tradeoff to reflect design constraints must be taken into account when evaluating the design as well. The optimal method of solving these conflicts and evaluating the merits of various trade-offs is in performing usability tests. However, usability testing is beyond the scope of the current document.

As effective as individual guidelines and local design rules may be in supporting a user-centered design of the cab, they may give an incomplete picture of how well designed the item is. Each component, which may have been designed using the rules agreed upon, is still part of a larger system. When all the components are put together, the system may be lacking from a human factors perspective. Interactions between various components may lead to unanticipated consequences. The optimal method for learning about these problems is through usability testing.

\subsection{GUIDELINES ORGANIZATION}

This document is intended to be used as a reference guide. Each chapter is self-contained and the reader is encouraged to go directly to the topic of interest, referring to the table of contents or the index to find specific topics of interest. Because the document is meant to be used as a reference, the reader will encounter some redundancy. Some topics will be discussed in more than one section.

Each chapter contains the same elements: a set of checklist items, human factors considerations, and operational aspects. The checklist items summarize the key human factors recommendations for treating a particular issue. The human factors considerations provide a background discussion of the human factors research contributing to individual guidelines. The operational aspects discuss the relevant operational issues specific to the locomotive cab environment.

The guidelines, as described in the table of contents, are organized into three sections: Environment, Layout, and Workstation Design.

- Environment addresses issues such as heating, ventilation, air conditioning, vibration, and noise that affect the overall 
working conditions in which the engineers and conductors operate.

- Layout address issues like control stand/console design, egress, visibility, and seating that define the engineer's physical relationship with the equipment in the cab and the outside environment.

- Workstation Design addresses topics such as controls, displays, auditory devices, and computer input.

These topics address how the engineer obtains information about the status of the locomotive and how train control is implemented.

\subsection{GENERAL PRINCIPLES}

The human factors guidelines presented in this document are representative of a small, but important set of human factors principles. These principles are listed briefly below:

- A systems approach acknowledges that the operators, machines, processes, and environments do not operate in isolation (Sanders and McCormack, 1993), but as part of an integrated whole. One of the guiding philosophies in this document is to adopt a systems approach to the design and development process of the locomotive cab. In designing or evaluating the individual components in the locomotive cab, we must recognize that they do not operate in isolation. Changing one part of the system may impact other parts.

- A user-centered design approach is used which means designing the system around the operator. This approach not only recognizes the limits of the human operator to receive, process and act upon information; but also recognizes the environmental and physiological factors that limit performance such temperature and humidity, noise and vibration, and anthropometric characteristics (Burgess, 1985).

A user-centered design also puts the operator in charge. This means that the locomotive engineer initiates actions rather than simply responding to changes in the state of the system.

- One of the goals of incorporating human factors principles into the design of the locomotive cab is to 
eliminate the likelihood of operator error. While this is a worthwhile goal, human error may never be completely eliminated. To address errors that do occur, the system should include features that minimize the impact of these errors. Designing error-tolerant systems involves a number of steps that include prompt detection and corrective action (Miller and Swain, 1987; Salvendy). It is important to give prompt feedback to tell the operator when an operator error or equipment failure occurs. Including redundant equipment that can take over when one piece fails, and can keep the entire system from failing, is also important. The system can be designed to monitor performance and tell the operator when the system is operating beyond a predetermined range. When an error does occur, the system offers simple and comprehensible methods to recover from the error (Shneiderman, 1992).

- An important part of the engineer's job is to process information. Using redundancy in coding information to increase the likelihood that the operator receives the information and giving feedback after the operator activates a control helps the operator to understand what the system is doing.

- Consistency is an essential element in developing ergonomically sound designs. Consistency in design facilitates learning and remembering how the system operates, reduces the likelihood of errors, and results in faster operation (Shneiderman, 1992). Locomotive cab designs should be compatible with how people organize information and act on their environment. Designers and evaluators should take into account population stereotypes and user expectations. 


\section{CHAPTER 2. KEY POINT SUMMARY}

This chapter lists the key recommendations and highlights from the following chapters. It can be used alone as a quick reference or checklist with the remaining chapters as a source of more detail on a specific item or general area. The reader should keep in mind that individual guidelines may sometimes conflict with one another. The relative importance of a given guideline will depend upon the context in which it is applied. In applying these guidelines, the evaluator or designer must weigh the trade-offs between these different design goals and economic or other constraints. It should be noted that the discussion of an item in this section (e.g., touch screens) does not constitute an endorsement of the item for use in the locomotive cab. It is intended only to provide guidance on implementation, if such a system is to be used in the cab.

\subsection{CAB ENVIRONMENT}

\subsubsection{Heating}

- The heater should maintain a minimum temperature of $64^{\circ} \mathrm{F}$, at a point 24 inches above the center of each seat, in cabs which can be occupied for more than 3 hours.

- The temperature from floor level to head level should not vary more than $10^{\circ} \mathrm{F}$.

- Cab glazing should be designed to reduce heat loss by radiation, conduction, and by air infiltration at poor seals.

- Cab floors should be insulated to prevent ambient heat loss and conduction from feet when standing; and walls should be insulated to reduce radiation.

- Sidewall convectors or other means to create a thermal barrier should be standard equipment rather than options which can be omitted.

- Cabs should provide a means to add humidity to the heated air to improve comfort, reduce skin and membrane drying, and aid dust settling. 


\subsubsection{Ventilation}

\subsubsection{Air Conditioning}

- Outside air should be provided at a minimum rate of $20 \mathrm{cu}$. $\mathrm{ft} . / \mathrm{min}$./person. Operations where the outside temperature is above $90^{\circ} \mathrm{F}$ require a minimum rate of $150 \mathrm{cu}$. $\mathrm{ft} . / \mathrm{min} . /$ person unless air conditioning is provided. An alternative standard is $1 \mathrm{cu}$. $\mathrm{ft} . / \mathrm{min} . / \mathrm{sq}$. ft. of floor.

- Air speed at the engineer's head should be adjustable either continuously or in three discrete increments from near 0 to $400 \mathrm{ft} . / \mathrm{min}$. Alternately, whole cab ventilation speeds of 0.5 $\mathrm{ft} . / \mathrm{min}$. (6.25 ft./min. maximum) for winter and $1.65 \mathrm{ft} . / \mathrm{min}$. $\left(8.25 \mathrm{ft} . / \mathrm{min}\right.$. maximum) for summer (above $86^{\circ} \mathrm{F}$ ) should be present.

- Outside air should be filtered to remove dust, insects and other debris. Intakes should be positioned to prevent introduction of fumes and vapors.

- Ventilation system noise should be controlled.

- Positive cab pressure should be maintained to reduce infiltration of outside contaminants and drafts.

- Air conditioning should be provided to maintain the cab temperature below $85^{\circ} \mathrm{F}$.

- The ability to reduce humidity is important in areas with high temperatures and high dew points.

- The temperature from floor level to head level should not vary more than $10^{\circ} \mathrm{F}$.

- The air conditioning system should not discharge directly on cab occupants.

- Windows should be provided with movable visors or tint shades to reduce radiant heat gain.

- Air conditioning should be used to decrease use of both open windows and ventilation systems and to reduce interior noise.

\subsubsection{Noise}

- A maximum continuous noise level of $75 \mathrm{dBA}$ is a desirable goal, but is well below the usual current cab levels. It is 
also well below the current $90 \mathrm{dBA}$ OSHA limits. The 90 dBA noise limit is a maximum which considers both comfort and practicality. However, continued exposure to acoustic noise in the $90 \mathrm{dBA}$ range can result in long-term hearing loss and discomfort to the crew. It is also likely to result in communication errors, particularly for train crews with existing hearing loss. Even a noise at the $80 \mathrm{dBA}$ level may restrict communication, but is not likely to damage hearing.

- Internal venting of air brakes can be an irritating noise source and can stir up dust in the cab. Internal venting of air brakes should be reduced. However, the sound is used by the train crew as a cue to judge brake operation and should not be completely eliminated.

- Air conditioning can reduce noise levels due to open windows and other external ventilation systems. Use of a ventilation system and open windows during warm weather adds significantly to noise levels. They can create noise directly and provide a clearer path for entry of outside noise.

- Insulation from exterior sound may hinder hearing of exterior noises that provide important cues (e.g., horn loudness, torpedoes). Use of exterior sensors and interior annunciators may be required to compensate.

- Active noise cancellation may potentially provide important benefits and complement current noise reduction methods. Combined with relatively low cost and installation ease, this evolving technique should be investigated.

\subsubsection{Toilet Facility}

- Toilet facilities should be designed carefully (e.g., avoid inside corners that create pockets, provide a floor drain) and should be made of appropriate materials (e.g., nonporous) to permit easy cleaning/disinfecting.

- Odors are the primary source of the perception of lack of cleanliness. Toilet designs that isolate waste-generated gases from the lavatory should be used to eliminate the largest source of odor.

- Better lighting will improve the visual impression of the facility. A two-stage system should be used to allow the 
user to select a lower level at night and a brighter setting during the day.

- The lavatory facility should be full height, tied in to the heating/air conditioning system, vented to the outside, and noise insulated. Hand holds should be provided for support when balance-disrupting motions occur. Ventilation and good door seals are important to prevent odors from entering the main cab. The door latch should be able to keep the door from flying open during severe train motion.

- Holding tanks should be located outside of the cab or be separately vented to the outside and not be permitted to exchange gases with the lavatory.

- There should be no sharp edges, corners or protrusions that could cause impact injuries.

- Steps leading down to the lavatory should not be so steep that they hinder entry.

\subsubsection{Vibration}

- "Locomotive crew may experience significant repetitive mechanical shocks and/or vibrations which are known to affect comfort, safety and health. The motions of interest are those along the vehicle's three translational axes $(\mathrm{X}, \mathrm{Y}$ and $\mathrm{Z}$ ) and roll motions about the vehicle's direction of travel. There is evidence that roll and lateral motions are particularly important with regard to crew comfort. Guidance for the evaluation of mechanical shock and vibration can be found in the ISO's (International Organization for Standardization) Document 2631. Emerging research suggests that repeated longitudinal shocks, such as those encountered in switching operations, may also negatively influence crew health."

- Muscles are used to overcome vibration effects on the body. This can produce fatigue and overuse syndromes, depending on the effort required and length of exposure.

- Position (standing versus seated) and direction of the vibration (vertical versus horizontal) are also important factors. In general, sitting is more stressful than standing while horizontal vibration is slightly more bothersome than vertical vibration.

- In terms of loss of comfort, the human body is sensitive to vibration in the 0.4 to $20 \mathrm{~Hz}$ range. With regard to vertical 
vibration, the area of greatest sensitivity between 4 and $10 \mathrm{~Hz}$, very low frequency vertical motions $(0.1$ to $0.5 \mathrm{~Hz})$ are not experienced as vibration but may result in motion sickness.

- Use of dampers at the seat post can reduce the vibration exposure to the operator. However, this can create a difference in relative vibration of the operator and the controls and displays. Operation and legibility problems can result if the difference is large enough.

- Active systems can provide greater vibration control than passive systems. They can be potentially applied to

\subsection{CAB LAYOUT} locomotive suspension, cabs, or seat posts.

\subsubsection{General Design}

- User population sizes should be used to design the cab with the male 95th percentile dimensions used to set clearances and the female 50th percentile dimensions used to set reach envelopes.

- Enough space should be allocated for each cab occupant: 65 sq.ft. is a minimum amount of floor space. Comfort facilities (toilet, water cooler, storage, refrigerator, etc.) should be located out of the main area of the cab and not counted as crew space.

- The height of the cab ceiling should be at least 76 inches (European designs use 79 inches).

- The toilet should be positioned so that light does not create glare or reflections for the engineer when the door is opened at night.

- Auxiliary items, such as a first aid kit, flares, torpedoes, and fire extinguisher, should be mounted where they are accessible, but do not impede movement in the cab.

- Changes in floor levels in the cab (e.g., raised seat platform) should be kept to a minimum to reduce tripping hazards.

- Equipment should not protrude into the open space unnecessarily, and sharp edges and corners should be 
eliminated.

- Interior surface finish should be light colored, of low reflectance and easy to clean.

- A separate panel should be provided for remote operation of consist locomotives. It should be located in a secondary position near the control stand.

- Cab design should consider ease of maintenance and cleaning.

- Equipment failures should be readily identifiable and only cause loss of function that the engineer can safely handle (failsoft). No single failure, or likely combination of failures, should create an unsafe loss of function (failsafe).

- Designing for extremely large individuals is appropriate when a design feature must accommodate most of the population (e.g., a doorway).

- Designing for an adjustable range is permissible when features can be easily tailored to the individuals who use them (e.g., seats, keyboards).

- Designing for the average individual is appropriate in noncritical situations, where designing for an extreme is inappropriate and where adjustability is impractical (e.g., a toilet seat).

- To provide good visibility while minimizing fatigue due to poor neck and head posture, regular viewing tasks should be within a 30-degree cone around the normal line of sight (Grandjean, 1988). The normal line of sight is 10-15 degrees below the horizontal plane. Displays should be placed within a viewing angle between 5 degrees above and 30 degrees below the horizontal plane in establishing the height of the seat in relationship to the windows and the visual displays in the cab.

\section{Controls and Displays}

- The primary displays and controls should be placed so that the engineer may view them without changing eye or head position from the normal line of sight.

- Controls and displays of secondary importance may be located so that eye movements are necessary, but head movements are not. 
- Non-critical displays and controls may be located outside the normal line of sight.

- The use of angled work surfaces should be considered when there are many controls and displays to arrange in the workstation. Controls on an angled surface allow placement of a greater number of controls within easy reach.

- The movement of the control (e.g., left, right, up, down, clockwise, counter clockwise) should be consistent with the movement shown on the display or with system response.

- Control size and spacing should permit the engineer to operate the controls without accidentally activating neighboring controls.

Work-space Envelope

Maintenance
- The controls should be placed so that the operator's hands do not have to reach frequently or be elevated above the shoulder for substantial periods.

- Padded forearm supports should be used to relieve pressure at the shoulder and elbow.

- Design the workstation so that the engineer's elbows remain flexed (bent) and allow for control activation.

- Sufficient clearance should be provided for the engineer's thighs under the work surface.

- Provide an adjustable work-surface height to fit individual physical dimensions and preferences.

- Consider providing a workspace that allows both sitting and standing.

\section{Labeling}

- Labels should be designed to survive wear and damage under normal operating conditions.

- Use words and abbreviations that are commonly known and meaningful to the user. 
- Instructions should be easily seen.

- Wording of labels or instructions should be concise.

- Labels should be located next to or on controls.

- Danger signs should use white lettering on a red background.

\section{Access}

- Workspace and access areas should allow adequate room for needed body motions such as crawling or kneeling.

- Those components most likely to fail and those most critical should be the most accessible.

- Make access panels accessible with common tools.

\section{Repair, removal \& replacement}

- Consider modular design and throw away units to minimize the skill level needed to maintain the equipment and reduce the impact on related sub-systems or components.

- Use self-adjusting mechanisms where possible.

- Use self-lubricating sealed assemblies with throwaway replacements.

- Make stored materials, assemblies and spare parts easily accessible.

- Clearly mark storage locations and parts.

- Make retaining or load securing devices easily removable.

- Make removable parts easily accessible.

- Make misconnection of parts impossible through use of keyed interconnections.

- Design gaskets and seals to be easily replaced without completely removing or disassembling other equipment. 
- Use quick disconnect electrical devices where units are replaced frequently.

- Use hinges, latches or catches to reduce handling and storage of covers.

- Design covers to avoid holding dust and dirt.

- Design cables or wire runs without bends and locate them to prevent stepping on them.

- Design both ends of cables and plugs to prevent misconnection.

- Route wiring away from lines that carry combustibles to prevent fires from sparking.

- Design items over 45 pounds for two-man operation.

- Enable use of common hand tools to repair or replace defective items.

- Minimize the number of special tools required. When they are required, make them captive to avoid being misplaced.

\section{Inspection and Testing}

- Provide quick and positive identification of malfunctions and components.

- Minimize the need for special test equipment.

- Design major assemblies to be completely inspected by means of removable housing.

- Reservoirs, gauges, meters should be visible without removing panes or other components.

- Visual access should be available for maintenance in progress.

- Make seals and gaskets easily visible after installation.

\subsubsection{Access}

- The placement of doors should consider post accident evacuation. Rear doors leading outside offer ready exit routes 
in most, but not all, accident scenarios. The piling of cars on and around the locomotive may in some circumstances block exits.

- Doors should open outward to permit easier pre-accident exits. This also eliminates the need for a clear area in the cab to allow an unobstructed inward swing.

- Nose doors should be offset to reduce cab drafts. Doors that directly access the front of the locomotive are susceptible to drafts and are less crashworthy.

- Door latches should be examined for potential hand pinch areas in their range of motion.

- The bottom edge of doors should clear the walkway they open over to reduce the obstruction by ice and snow.

- The nose door should include a small sight glass to see if there is somebody that could be struck when opening the door.

- A wide opening or pop out side window or $25 \times 25$ inch roof hatch may be desirable to provide an additional evacuation route.

\subsubsection{Visibility}

- Visibility requirements should be determined by the objects that must be seen and the human and train reaction lags in the control actions that the objects trigger.

- The windows should permit the operator to see a track level object as close as 50 feet away, and an overhead object (e.g., signal bridge) as close as 55 feet away. Lateral field-of-view should be at least $180^{\circ}$ and preferably $220^{\circ}$.

- Too much window area can have drawbacks. Examples are radiant heat gain, heat loss, glare, reflections, vulnerability to thrown rocks, and now, gunshots.

- Accommodate the engineers visual requirements in both directions if the locomotive will operate in both directions. Use of a long haul locomotive for switching or long hood forward operation does not fully meet the engineer's visual requirements. Properties that use bidirectional travel for long periods or eliminate switchers for financial or other reasons 


\subsubsection{Seating}

- Locomotive seats should be cushioned at least 3 inches thick, use the buttocks for primary support, exert little pressure on the thighs especially at the front edge, support the lower back and have arm rests 4 inches wide and 13 inches long.

- Seat height should be adjustable from 16 to 19 inches in steps no larger than 1 inch. Seat should adjust forward and back at least 4 inches from the 50th percentile position.

- The seat cushion should be contoured for buttocks and the back for spinal curves in order to even pressure and provide support. Provide adjustable lumbar support to increase support and comfort level.

- The seat's backrest should recline between 95 and 115 degrees, the seat pan should tilt back between 1 and 5 degrees from horizontal, front edge higher.

- A backrest curved on a radius of 18 to 24 inches or with lateral support will help during side sway.

- A rectangular seat pan with elevated sides is preferable to a round seat pan. The lateral support offered reduces muscular effort in curves or lateral cab movements and accommodates legs when spread at a comfortable angle.

- A continuous balance seat pan may correct this by tilting to relieve spinal pressure when leaning. The need to lean forward to operate controls and twist and lean to look out side windows may negate the best conventional seat design because it creates strain on spinal discs.

- The ideal seat adjustment mechanism is easy to use, reliable, and wear resistant. A swivel may be needed to access the seat and to accommodate the need to turn to look to the back and sides. The seat covering should be made of fabric or perforated leather to reduce perspiration and heat buildup.

- Non-seat characteristics can have a direct or indirect impact on the seated position or use of the seat and need to be considered to determine seating comfort. Non-seat factors include: leg room, knee room, availability of footrests, clearance from sidewall, vibration levels, ease of entry/exit, clearance when 


\subsection{WORKSTATION DESIGN}

swiveling, visibility, and reach-to-control distance.

\subsubsection{Controls}

- Place motion controls directly in front of the engineer with the brake module on the left.

- Place the radio hand controls on the left hand side to allow an engineer to operate the locomotive motion controls with his right hand while using the radio with his left hand.

- Controls for the sanders, whistle, horn, headlights, radio and microphone should be located within the zone of reach and preferably within the zone of comfort, if possible.

- Controls should be arranged to minimize engineers changing their position solely to operate a control. Position all controls so that, in manipulating them, operators do not appreciably move their nominal eye reference and possibly miss seeing important events occurring outside or on the principal internal display (Woodson, 1992).

- Controls should be arranged according to the order they are expected to be used. Tracing the sequence of control use will help identify poor arrangements.

- Controls should be consistent with normal limb motions. This means that where arm motions are needed they should be forward and back, not sideways.

Controls that have a similar function or purpose should be grouped together.

\subsubsection{Electromechanical Displays}

- If displays are ordinarily read in sequence, arrange them in order, either horizontally or vertically.

- When the circumstance includes an array of displays that must be monitored on a regular basis to determine whether conditions are approximately "normal," arrange the displays so

that all the pointers are aligned alike when the instruments are indicating normal operation.

- Arrange the normal pointer reading at the twelve o'clock 


\subsubsection{Auditory Devices}

position when the array is vertical and at the nine o'clock or three o'clock position when the array is horizontal.

Make lettering clearly legible. The American National Standard for Human Factors Engineering of Visual Display Terminal Workstations (ANSI/HFS-100, 1988) gives recommendations for letter size, word spacing, viewing distance, contrast ratios, brightness ranges and other critical parameters.

- Provide the vigilance detector system with a time constant that varies based on both speed and control activity.

- Provide additional displays associated with a warning advisory panel that indicate which locomotive in a multiple consist is experiencing a particular problem.

- The panel should contain an end-of-the-train unit alarm and a built-in lamp test function.

- Non-speech signals should be in the 200 to $5,000 \mathrm{~Hz}$ range and ideally in the 500 to $3,000 \mathrm{~Hz}$ range. Loudness of sounds used should be consistent with the ambient sound level, but not so loud that they startle or disrupt the proper response. Loudness should also be consistent with the urgency of the message.

- For the vigilance system, the audio alarm and visual alert should be near the windshield since the engineers attention should be directed towards the outside. For the engine monitoring system, the warning sound should come from somewhere near the warning advisory panel.

Avoid the use of sounds that could be confused with operational or malfunction noises (e.g., air brake releases, pump operations, sand discharges, etc.). Limit the selection of advisory sounds to no more than four to ensure proper identification.

\subsubsection{General Principles}

\section{Dialog Design}

- Avoid actions that surprise the engineer, require tedious sequences of data entries and make obtaining necessary 
information difficult.

- Include features that give feedback regarding actions taken and system responses with easy reversal of actions. Actions should be initiated by the engineer.

- Consistent sequences of actions should be required in similar situations, identical terminology should be used in prompts, menus, and help screens. Use consistent commands whenever possible.

- Minimize display clutter to reduce short-term memory load.

Menus

Function Key Menus
- Make the organization of the menu or user interface as transparent or obvious as possible to the user to speed the search for information.

- Increase the menu breadth to the maximum practical level for linearly organized lists such as alphabetized lists, months of the year, letters of the alphabet.

- When there is no inherent linear ordering of alternatives, the optimal number of alternatives will fall between three and twelve.

- Limit menu trees to three levels to reduce the likelihood of getting lost or disoriented, where possible.

- Provide rapid access methods by which the engineer can enter a series of multiple menu selections at once or go directly to a given menu.

- Standardize the location of information elements to aid the development of expectancies about where that information is located and decrease response time.

- Provide enough functions keys to support functionality, but not so many that scanning and finding are difficult.

- Arrange keys in groups of three or four based on semantic relationships.

- Use space, size, and color to distinguish groupings. 
- Place function keys that have a destructive effect away from the main keys to avoid accidental activation.

- Color code keys to be consistent with user expectations (i.e., green for go, red for danger or warning).

- Label keys clearly and distinctively.

- Adopt a consistent abbreviation rule such as truncation or vowel deletion when abbreviations are necessary.

- Place the most frequently used keys in the most accessible positions.

- Minimize the use of qualifier keys (i.e., Shift, Alt, Command, Control).

- Preserve spatial relationships between soft function key labels on the screen and generic function keys on the keyboard.

- Use the same coding schemes (space, size, grouping) for both the displays and controls.

- Be consistent in function key assignments across screens and subsystems. When a function key performs different functions in different operational modes, assign equivalent or similar functions to the same key.

- Give the user feedback regarding the status of function key operation. When function keys are pressed, the system should give the operator feedback to indicate whether some action took place and how the system is responding.

- Inactive key or menu selection should be grayed out or deleted to indicate whenever a particular menu selection is inoperative.

- Use a status indicator to indicate what mode the keys are in if there are special keys that have different modes.

- Enable easy access to the home menu in menu systems with multiple levels so the user may return quickly to the home menu.

- Response time should be appropriate to the task. For unusually fast or slow response times, the system should give 


\section{Error Management}

\section{Alarm Design}

- Avoid response times longer than 15 seconds. Extreme variation in response time should be avoided where possible and acknowledged by the system where it is not possible.

- In managing errors, the first goal is to prevent their occurrence. The system should be designed to prevent the engineer from committing errors wherever possible. For example, in locomotives with $\mathrm{AC}$ traction motors, putting the throttle into reverse when it is moving in the forward direction will damage the motors. The system could prevent this from happening by not allowing the engineer to execute this command when this condition occurs or delaying its execution until the conditions are appropriate.

- Where the engineer must engage in operations that are difficult or impossible to undo (e.g., destroying data), the system should notify the engineer and require confirmation before executing the command (Smith and Mosier, 1986).

- Enable the user to recover from errors quickly and easily, (Norman, 1991) when they occur. A responsive system will indicate the nature of the problem and suggest how to correct it.

- Provide an undo function to reverse actions or correct an error. Allow engineers to undo a sequence of commands to change previously entered selections.

- When a command must be reentered to correct an error, prompt the user to reenter that portion of the command that needs correction. Do not require the entire command to be reentered.

- Do not overload the user with too many alarms. This may distract the engineer.

- Auditory alarms are preferable under the following conditions:

the message is short and simple the message will not be referred to later the visual channel is overburdened the message deals with events in time 
continuously changing information is presented, and vision is limited.

- Alarm design should convey the urgency or priority of the warning so that the engineer can allocate his or her attention appropriately, particularly if multiple warnings are present.

- Give the engineer control over alarms by providing a means to acknowledge and turn off non-critical alarms.

- Where alarms are presented by both visual and auditory methods, allow the engineer to turn off the auditory alarm without erasing the visual message that accompanies the auditory signal.

Feedback and User Guidance

\section{Error Messages}

- Feedback should provide some indication of system status to users at all times and occur close in time to a related event.

- Feedback should clearly distinguish between the system that is waiting for the user to initiate an action and a delay in the response to an action initiated by the user.

- Indicate clearly the currently selected mode when the results of user action are contingent upon different operation modes.

- Every input or response selection by the user should consistently produce perceptible response output from the system.

- When errors occur, feedback should clearly indicate the corrective action to take.

- Error messages should be specific (task oriented) and as concise as possible and written from the user's perspective. Use language that the engineer will understand.

- Abbreviations should be consistently displayed in the visual format and placement, and in grammatical form.

- Use active voice and a positive tone to tell the user what needs to be done.

- Consider multiple levels of messages where users may desire more detailed levels of information or where more than one error was made. 
- Display the error message after the user completes a response to minimize disruption of the user's task performance and thought processes.

- Display error messages within 2-4 seconds following the response for which the error is detected.

\subsubsection{Automation}

- The engineer must be in command. Since the engineer is responsible for the safe operation of the locomotive, he or she must have the ability to control those operations.

- The engineer must be an active participant and informed. To command effectively, the engineer must have an active role in controlling the train. This means engaging in relevant activities so that situational awareness is retained.

- Automated systems must be predictable. The engineer must be able to predict how the locomotive will be affected by the automation to know how to use it, and it must behave in a consistent manner to know when a failure occurs.

- Each element of the system must have knowledge of the others' intent. The human operator must be able to monitor the automated system. Automated systems must be able to monitor the human operator.

- System operation should be easily interpretable by the operator to facilitate detection of improper operation and diagnosis of malfunctions.

- Design the automation to perform the task in a way familiar to the user. When the automation fails, it should be announced so that the operator takes over active control of the system.

- Design the automation to prevent peak levels of task demand from becoming excessive.

- Design control automation to be of most help during times of highest workload and somewhat less help during times of lowest workload.

- Provide meaningful duties to maintain operator involvement and resistance to distraction if automation reduces task 
demands to low levels.

- Control automation should provide the engineer with an appropriate range of control and management options.

- Provide a way to check the setup and information input to automatic systems.

- All displays should contribute to and maintain situational awareness.

- Displays that have multiple modes should clearly indicate what mode is active.

\subsubsection{Electronic (Computer Generated) Displays}

\section{General}

Hardware
- Provide a nominal viewing distance of 20 inches $(510 \mathrm{~mm})$ and all areas of the display be viewable from within 30 degrees of the horizontal axis centered on the screen.

- A minimum screen recommendation is a $5 \times 7$ dot matrix, with $7 \times 9$ preferred. For environments in which symbols will be rotated, the minimum dot matrix is $8 \times 11$, with $15 \times 21$ preferred.

- To adequately see both foreground and background, it is recommended that the luminance of the foreground or background be at least $35 \mathrm{~cd} / \mathrm{m}^{2}$. The display should be capable of a minimum contrast ratio of $3: 1$ with $7: 1$ preferred.

- Provide controls to adjust the level of brightness and contrast to help the engineer adapt to changes in illumination.

- The refresh rate for a display should be at least $60 \mathrm{~Hz}$ per second for a screen with a dark background and light foreground (positive polarity). The refresh rate for a display should be at least $90 \mathrm{~Hz}$ per second for a screen with a light background and dark foreground (negative polarity).

- Eliminate glare from the environment. Surfaces should be painted in a dark color with a matte finish. Provide shades, visors, or tinting for windows. Orient the display to avoid reflections and consider the use of a hood to shield the display. Consider using anti-glare treatments such as neutral density filters and etched display surfaces where glare cannot be 
removed.

- To preserve dark adaptation, the engineer's display should be designed to be used at the dimmest setting possible while still maintaining good image quality.

- Displays that use incandescent lighting should incorporate dual lamps for redundancy. Bulbs should be replaceable from the front panel without the use of tools.

\section{Software}

Information Placement
- Information density generally should not exceed $25-30 \%$ of the active screen area. As the density of information increases, time and errors in task performance increase.

- The need to minimize information should not occur at the expense of clarity or task performance.

- The choice of abbreviations should be made on the basis of what engineers are likely to understand. Frequently used abbreviations schemes include: truncation, alphabetical, and abbreviations commonly recognized by the user population.

- Give engineers only as much detail as they need. Software should enable engineers to control the amount of information displayed.

- Use white space to separate information into columns with headings. Column headings save space by reducing the number of times that related items are repeated.

- Adopt a consistent format for all screens. Consistency allows user to develop expectancies about where to find the information, making it easier to learn how to use a new application.

- Place each design element in a consistent and specified location. This includes: screen titles, messages, alarms, commands, function keys, status displays, etc. Reserving specific areas of the screen for particular elements will aid in memorizing their location.

- Place items that need to be found quickly in the upper lefthand corner of the screen and items that are not time-critical in the lower right-hand corner. Visual scanning patterns take the 
form of a $\mathrm{Z}$ shape by beginning in the upper left-hand corner and ending in the lower right.

- Display critical information in the center of the screen, not along the edges, to prevent distortion due to parallax.

- Information will also be detected most quickly if it is within the area where visual acuity is best. This area is represented by a cone extending from the normal line of sight within an angle of 15 degrees.

- Group items that belong together.

- Grouped items should subtend no more than 5 degrees of visual angle (i.e., 12-14 characters by 6-7 lines) to facilitate search time. However, legibility and comprehension should not be sacrificed to meet this guideline.

Information Coding numbers that are critical or need to be read quickly.

- Where readability is important, flash coding can be implemented by using a separate blinking symbol placed near the information that must be read.

- When reverse video is used, leave a buffer zone on either side of characters and symbols to avoid legibility problems caused by the characters bleeding into the background.

- If size coding is used, use no more than two widely different sizes.

- Visual displays should be first designed to meet human performance criteria under monochrome conditions. Color coding should be a redundant coding technique since some 
engineers may have color vision deficiencies.

- Provide sufficient luminance contrast (brightness) when optimal color contrast is not feasible.

- Avoid using color in areas that are not in the engineer's direct line of sight, since it is difficult to discriminate colors in peripheral vision.

- The number of colors should be limited to less than six when the task requires discriminating between colors. Use no more than 7 colors for the entire sequence of displays and 4 colors in an individual display.

- Avoid the following color combinations: red/blue, red/green, blue/yellow, and green/blue.

- Color coding should be consistent with user expectations for the task (i.e., green for go and red for stop).

- Consider the use of color to format related items in a densely packed display.

Text Based Information (Typography)
- Complex or ornate typefaces (i.e., script or fancy block letters) should be avoided.

- Proportional typefaces which vary the width of individual characters and the spacing between characters are generally preferred to non-proportional typefaces.

- Use mixed case for presenting blocks of text, such as system messages and train orders.

- Upper case is appropriate for single words or short phrases which must attract the engineer's attention. However, to be effective, this technique should not be overused.

- Use white space to organize alphanumeric text.

- For interline spacing, the space between the bottom of one line and the top of the characters on the line below should be equal to or slightly greater than the height of the characters themselves. This represents a ratio of letter height to total space between lines of 1:2 to 1:2.7. 
- Use short familiar words and a simple sentence structure.

- When sentences describe a sequence of events, they should be described in the order in which they will occur.

- Sentences should also use the active voice since they are easier to understand.

- Express ideas in positive terms since negatively expressed ideas take longer to understand.

- Keep phrasing short and concise as possible. Avoid using words that have multiple meanings.

- Short words tend to be more familiar and easier to understand. However, a long familiar word is preferable to a short, unfamiliar word. While short words are generally better than long words, avoid using contractions.

- When abbreviations are used, choose those abbreviation that are commonly recognized and do not abbreviate words that produce uncommon or ambiguous abbreviations.

\section{Graphic Information}

\section{Symbol Design}

- Limit the number of symbols or geometric shapes used to represent objects to between 10 and 20 .

- Make the symbol or icon as representative as possible. The more directly the symbol conveys what the object or action represents, the more likely the engineer will understand. 
- Keep the symbol simple. Unnecessary embellishments add screen clutter and may confuse the user. However, symbols should not be so simple that they are confused with one another.

- Use symbols that the engineer is familiar with. Familiarity will reduce learning time.

- The shape of the symbol should be clear and unambiguous. Screen resolution should be adequate to discriminate between different symbols.

- Add a label to increase comprehension. The ability to comprehend and learn symbols can be improved by attaching a label.

- Create consistent shapes through limiting the use of angles, line thickness, shapes and the amount of empty space.

\subsubsection{Computer Input Devices}

- Design dimensions such as shape, size, and material must be compatible with human anatomical and physiological characteristics.

- Keep the number of controls to a minimum.

- Design control movements to be natural for the operator.

- Design the controls to provide enough resistance to prevent their activation by mistake or through vibration.

- Design the controls to withstand misuse.

- The control should give feedback so the engineer knows when it is activated.

- Frequent switching between input devices must be avoided.

\section{Keyboards}

- Keyboard height should be adjustable by the controller within a range from 23 to 32 inches (i.e., it should be possible to raise and lower the surface that supports the keyboard). The keyboard should be less than $30 \mathrm{~mm}$. thick from its base to the 
home row of keys, and its slope should be adjustable between 15 and 25 degrees from the horizontal.

- Alphanumeric keys should be logically arranged. If extensive numeric data are to be entered, a separate numeric keypad should be provided, visually distanced from the main keyboard, and arranged in a $3 \times 3+1$ matrix with zero (0) centered on the lowest row. To reduce syntax error, function keys should be provided for frequently invoked commands.

- Function keys should be clearly labeled to identify their functions, and these functions should be consistent throughout the system.

- Nonactive keys should be blank; mechanical overlays should not be used to restrict access to nonactive keys.

- The key used to initiate a command must be clearly labeled "Enter."

- Keyed data should be quickly displayed or "echoed" on the screen.

- Tactile feedback should verify keystrokes and inform the controller when the next action may be initiated. In designing tactile feedback, consider whether the user will be wearing gloves.

- The keyboard should be located a comfortable distance from the controller and directly in front of and below the associated visual display. Forearm and wrist support should be provided to reduce discomfort.

- Keyboards must be readable under all operating conditions.

- Guards should be considered for any key that would present a problem if inadvertently activated.

- To avoid increasing engineer workload, it is important to minimize requirements for keyed data entry, particularly for keyboard data entry in a moving train.

\section{Touchscreens}

- The sensitive areas of touchscreens should be large enough to permit activation by fingers if the land-on strategy is being used. The sensitive area should also be large enough to 
accommodate the use of gloves.

- Parallax caused by the curvature of the CRT screen should be minimized by using touchscreens mounted as close as possible to the CRT surface.

- Touchscreen displays should have sufficient luminance to be read easily under all operating conditions.

- A positive indication should be provided within about $100 \mathrm{~ms}$. to acknowledge activation.

- As with keyboards, touchscreen displays should conform to applicable minimum standards for dimensions, separation (of active areas), and resistance.

- Touchscreens should not be used if the task will require engineers to keep their arms up and unsupported for lengthy periods of time. 


\section{CHAPTER 3. CAB ENVIRONMENT}

This chapter examines the general locomotive cab environment aspects that affect crew comfort and performance. Although the aspects are treated individually, there is a great deal of interaction between them in causes, methods of correction, and the effect on the crew. Thus, an integrated design is needed to improve the cab conditions.

There is considerable evidence that environmental conditions affect stress levels and job performance (especially vigilance), but the nature of the effect can vary. Conditions outside of a perceived comfort level can cause discomfort which increases stress. Conditions which are too comfortable may reduce performance because of a lack of environmental stimulation. A moderate stress level may produce the best performance. During low workload conditions, a small amount of discomfort may enhance performance, but under higher workloads, the discomfort can distract and cause errors. Rapid transitions from low to high workload or from high to low may be distracting to the operator's level of situation awareness, while an overly comfortable environment may reduce performance. It is not the best practice to produce discomfort with the intention of producing the beneficial arousal since the opposite result can occur. Figure 3-1 shows some general guidelines for acceptable working

\subsection{HEATING} environments.

\subsubsection{Operational Issues}

Locomotive cabs are heated by either forced hot coolant (most common) or electric resistance. Heaters are installed at workstations for the engineer and other crew members with options offered for sidewall convectors (Jankovich, 1972). The sidewall convector provides a warm air barrier between exterior walls and occupants to reduce the radiant loss to cold walls. In a review of the recently introduced SD-60M Wide Cab (Riley et al., 1991), engineers mentioned installation of sidewall convectors when asked about improvements, showing that this is still an infrequent addition even in the latest cabs. 


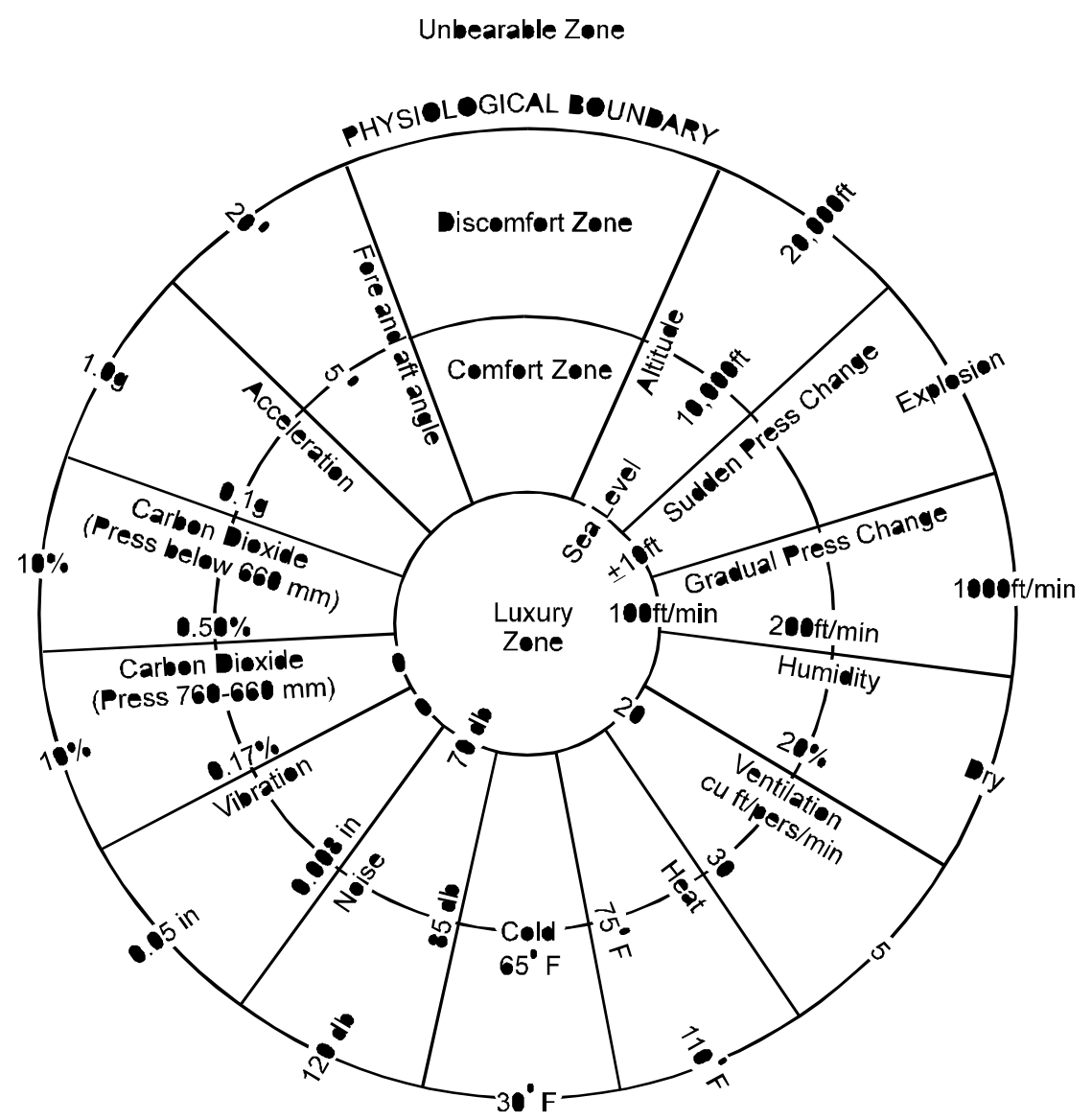

Figure 3-1. Work Environment Comfort Zones (Adapted from Human Factors Design Handbook, 2nd Edition, Woodson, et al., 1992, p. 755. Used by permission from McGraw-Hill, Inc., New York, NY.)

Gamst (1975) states that windows are opened in the winter for fresh air and to let the engineer lean out during switching (in this position the engineer's feet often contact the heater). Jankovich (1972) notes that locomotive heating systems are not designed to provide fresh air exchange. Thus, opening windows is the only way to provide fresh air, which adversely impacts cab temperature in the winter.

The relatively fixed position of the engineer during work limits the body's main ways to increase body warmth: waste heat from muscular activity (shivering excluded), moving away from cold areas to warmer ones, and increased blood circulation. The fixed position can also lead to limb stiffness if muscles become cold. The result is that the fixed working position limits an engineer's normal reactions to eliminate discomfort from cold if heating is insufficient. 


\subsubsection{Human Factors Considerations}

\section{General}

Evenness

Humidity
The three considerations for determining the proper level of cab heat are: 1) temperature level, 2) temperature evenness, and 3) humidity level. Exposure to low temperatures tend to have a physical effect before a mental effect. The initial physical effects such as shivering and a feeling of discomfort occur before mental effects show. Over time, the discomfort can become distracting (resulting in short-term memory deficits), and shivering and limb stiffening can hinder control inputs and reaction time. Uneven heat distribution can make comfort difficult to achieve, particularly if the limbs become cold. Blood circulation volume in the extremities is reduced in reaction to cold as a primary defense from heat loss during relative physical inactivity. This means that cold hands and feet will remain that way.

Jankovich (1972) measured locomotive cabs used in that period and found that the heaters' design and arrangement provided adequate heat level and evenness. However, the Canadian National Railways (quoted in Jankovich) and a study by Michaut and McGaughey (1972) found that air drafts made the heat inadequate. Higher speed operations worsen the effects of drafts caused by leaks at windows and doors. Cabs with front doors that exit directly or locomotives operating with the long hood forward are most vulnerable to this problem.

Loss of heat through window glazing, cold walls and opened windows for fresh air can create cold spots. The fixed position of the engineer does not permit him or her to turn from the cold to even out body temperature. The German Railways (quoted in Jankovich) found that a lack of floor insulation created cold spots and cooled the feet, especially when standing. Engineers will feel uneven heat in these conditions.

The heating of cold cab air to produce heat takes low relative humidity air and further lowers its relative humidity. The low humidity affects the effective temperature. The American Society of Heating, Ventilation, and Air Conditioning Engineering (ASHVAE) has conducted extensive research which shows that relative humidity is directly related to effective temperature. For a given temperature, a higher humidity makes it feel warmer while a lower humidity feels cooler. Low humidity also can directly impact comfort and health. The increased rate of evaporation caused by low humidity dries the skin, eyes, nose, and throat. 
The result is a feeling of dryness, chapping, and dried mucous membranes with reduced disease resistance and dust filtering ability.

Wilde and Stinson (1978) detail the health and comfort impacts of low humidity and make another interesting point. Dust particles normally absorb moisture, which increases their weight and speeds settling. Low humidity prevents this absorption and allows the heater fans to circulate more dust. The desirable relative humidity level varies with temperature and personal taste.

The ability to defrost windows is often a problem (Jankovich, 1972; Robinson, 1978; Wilde and Stinson, 1978). Increased humidity causes condensation/frost problems on windows under extreme cold conditions. Therefore, the ability to control the level of humidification, whether by humidistat or turning the humidifier on or off at will, would be beneficial.

\subsubsection{Recommendations}

\section{Temperature}

\section{Evenness}

Jankovich (1972) quotes the International Union of Railroads as recommending that the heaters preferably maintain $64-68^{\circ} \mathrm{F}$ with an absolute minimum of $59^{\circ} \mathrm{F}$. During informal discussions between the FRA and the Brotherhood of Locomotive Engineers (BLE) in December 1993, the BLE suggested a minimum heat requirement of $64^{\circ} \mathrm{F}$. MIL-STD-1472D recommends that vehicle heaters maintain a temperature of $68^{\circ} \mathrm{F}$ when the vehicle is occupied for more than three hours and arctic clothing is not worn. It also specifies a reference point where the temperature should be measured (a point 24 inches above the center of each seat). Based on the work constraints, on an engineer's reactions to cold, and these comments, the design should aim for a higher temperature level than the minimum AAR and FRA standard of $50^{\circ} \mathrm{F}$, and a $64^{\circ} \mathrm{F}$ would be more desirable. This is below the $68^{\circ} \mathrm{F}$ given in literature for a "shirtsleeve environment."

The evenness of heat is another important comfort consideration. MIL-STD-1472D recommends no more than a $10^{\circ} \mathrm{F}$ difference over any part of the body. Radiation of body heat to cold walls and floors or through glazing can make an occupant feel cold even at a proper air temperature. Sidewall convectors or other thermal barriers can help correct this radiation loss and should be standard equipment rather than an option. Floor and wall insulation and thermopane (and possibly low-E) glass can further reduce 
conduction and radiation losses of body heat. Drafts at windows and doors should be eliminated with good seals and offset nose door designs.

\section{Humidity}

\subsection{VENTILATION}

\subsubsection{Operational Issues}

\subsubsection{Recommendations}

Volume and Speed
The need for humidity control to provide comfort while heating is documented by ASHVAE, MIL-STD-1472D, and Wilde and Stinson (1978). A range of $30 \%$ to $70 \%$ is recommended. The current lack of a means to add humidity while heating the locomotive cab should be addressed. The humidifier could be integrated with the heating system or installed as a separate unit.

Open side windows, and in some cases open doors, are the only ways to provide fresh air to the locomotive cab. Generally, the open window is beside the engineer, which also allows leaning out as a means for him or her to look back down the train. This is a low cost way to provide adequate airflow when the locomotive is moving at a good rate of speed.

The open window allows dust and particles to enter the cab. This material settles throughout the interior and can contaminate equipment and increase maintenance needs and can injure the crew members. Wilde and Stinson (1978) found that eye injuries were the third most common railroad injury (13\% of total in 1976 Canadian National Railways data) and the predominant cause was entry of foreign objects through open doors and windows. The open windows also provide an unobstructed path for noise to enter the cab. Finally, any security (ballistic protection) offered by the glazing is negated when the window is open and the engineer is seated next to the open area.

A cab with a dedicated ventilation system that allows ventilation with the windows closed is desirable. MIL-STD-1472D recommends outside air be provided at a minimum rate of $20 \mathrm{cu}$. $\mathrm{ft} . / \mathrm{min} . /$ person and at a minimum of $150 \mathrm{cu}$. $\mathrm{ft} . / \mathrm{min} . /$ person for operations without air conditioning where the outside temperature is above $90^{\circ} \mathrm{F}$. Woodson (1990) recommends $1 \mathrm{cu}$. ft./min./sq. ft. of floor space.

Jankovich (1972) quotes the International Union of Railroads recommendation of general cab ventilation speeds of $0.5 \mathrm{ft} . / \mathrm{min}$. 
Noise

\section{Air Quality}

Drafts

\subsection{AIR CONDITIONING}

\subsubsection{Operational Issues}

(6.25 ft./min. maximum) for winter and $1.65 \mathrm{ft} . / \mathrm{min}$. $(8.25 \mathrm{ft} . / \mathrm{min}$. maximum) for summer. MIL-STD-1472D presents a different measurement reference point and speed. It recommends air speed at the head be adjustable either continuously or in three increments from near 0 to $400 \mathrm{ft} . / \mathrm{min}$.

It is worth noting that the lack of air conditioning places more stress on ventilation speed and volume, with an increase of noise if fans are used. Thus, an advantage of air conditioning is that it can reduce the flow rate performance requirements of the ventilation system.

Ventilation systems generate noise from the fans (open windows allow all outside noise in and can add wind noise). Proper design of the fan blade and vent locations can reduce this noise. Ventilation systems that do not raise noise levels above the maximum level are preferable to an open window.

Ventilation air should be as clean as possible. The air in the railroad environment can be laden with dust and fumes. Intakes should be positioned to reduce intake of such contaminants. Air should be filtered to remove dust, pollen, insects, and other debris. Interior brake venting which introduces odors and other contaminants from the brake air system and which can stir up settled dust should be avoided.

Whether moving air is perceived as a pleasant breeze or a draft depends upon variable environmental and personal characteristics. Therefore, a ventilation system should be as adjustable as possible with louvers at ducts to vary flow paths and fan controls or damper adjustments to adjust speed. A closed cab can provide a positive inside pressure which can reduce drafts from outside leaks. An additional benefit is a reduction in dust infiltration which can improve cab cleanliness and lessen equipment contamination.

Locomotives operate through various climates which include the high summer heat and humidity. Locomotives are also exposed to the direct sun much of the time. This heats the metal locomotive body and heat passes through the glazing partially as infrared rays. Thus, the summer heat conditions experienced in the cab can be as extreme as the cold of the winter.

Air conditioning is an option available on locomotives, but its use 
is a source of some controversy. Some see it as valuable while others see it as too costly. The result is that it is installed on less than half of operating locomotives, primarily those that operate in the warmer areas of the country.

There are two areas of concern with air conditioning: initial cost and maintenance cost. Air conditioning adds significantly to the cost of a new locomotive. When installed, it is a source of maintenance problems and has a lower than desired reliability. Maintenance work on air conditioning may have to be contracted out because of specialized equipment and skills needed. This makes the repair process more complicated and may extend locomotive down time.

At the informal discussions between the FRA and the Brotherhood of Locomotive Engineers (BLE) in December 1993, the BLE gave the air conditioning issue the highest priority. Rockwell and Kiger (1989) and Riley et al. (1991) conducted studies which found that air conditioning was widely desired by engineers. This finding is supported by Jankovich (1972) who states that standard air conditioning is the ultimate solution to cab ventilation. Riley et al. recommend air conditioning for proper comfort.

\subsubsection{Human Factors Considerations}

\section{Feeling Hot}

Discomfort caused by heat, unlike cold, affects mental activities before physical ones. Losses in dexterity, higher intellectual functions, and alertness (ranging from subtle to marked) can be expected. Engineers' need for vigilance and the mental aspects of train force planning make these losses a pertinent consideration. A study by Ramsey et al. (1983) of safety-related errors (unsafe behaviors) in industrial settings showed a rise in safety errors began at about $74^{\circ} \mathrm{F}$ and increased with temperature, particularly at high workload levels. Figure 3-2 depicts the relationship found between temperature and unsafe actions. The safety errors noted included shortcuts and impromptu changes in work procedures. This finding has great bearing in the context of the railroad work rule environment where judgment and mental function play a great role.

There are several things that add to the thermal load on the engineers. The outside temperature, humidity levels, and radiant heat gains can affect comfort levels alone or in combination. High levels of humidity reduce the evaporation rate of sweat, the body's primary means of losing heat. While increasing ventilation rates 
can compensate to some degree, there is a limit to the improvement. Also, the accumulation of wetness that can occur is an additional source of discomfort. If the wetness is at the seat contact area, it is more uncomfortable.

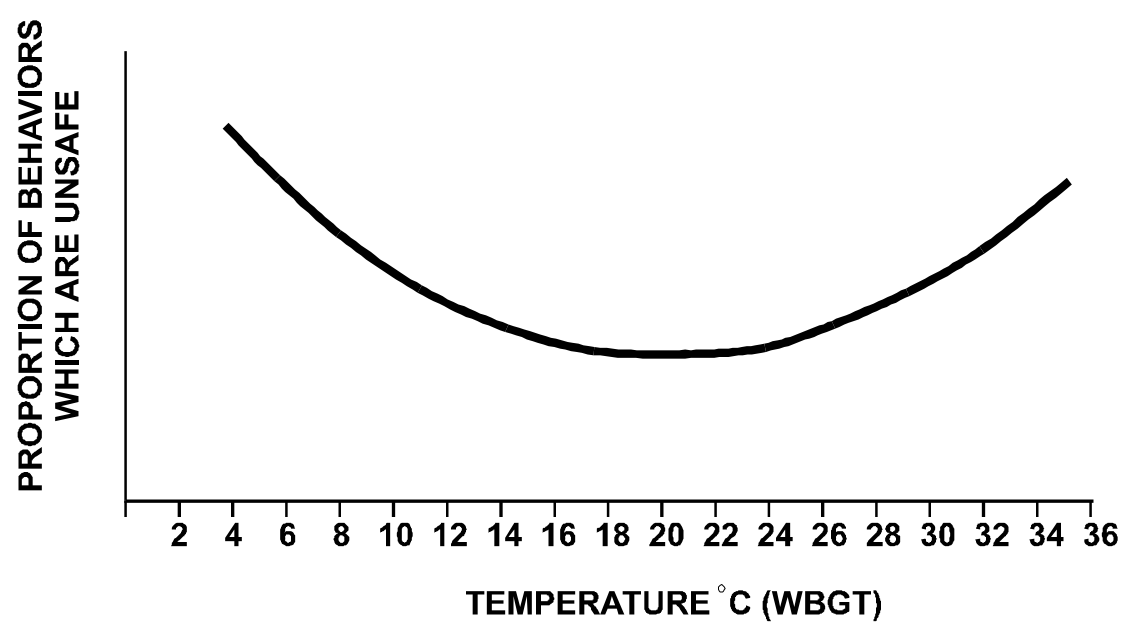

Figure 3-2. Relationship between Temperature and Unsafe Behaviors (Reprinted from Journal of Safety Research, Vol. 14, Ramsey, Jerry, D, et al., Effects of Workplace Thermal Conditions on Safe Work Behavior, 1983, p. 112, with kind permission from Elsevier Science Ltd., The Boulevard, Longford Lane, Kidlington OX5 1GB, UK.)

Radiant heating can occur from sunlight entering through the windows and from sun-heated cab walls and roof. The American Society of Heating, Refrigerating, and Air Conditioning Engineers (ASHRAE) notes that people are most sensitive to discomfort from warm ceilings (in a comparison of exposure to hot and cold walls and ceilings) even at small temperature differences. The International Standards Organization, in the annex to ISO Draft International Standard (DIS) 7730 (1992), sets a guideline limit of a $9^{\circ} \mathrm{F}$ difference in temperature of the ceiling surface compared to a point 0.6 meter above the floor in recognition of this result. The intent of this is to prevent the heating of the body by the warm ceiling. The relatively low ceilings in cabs make this effect a greater concern.

\subsubsection{Recommendations}




\section{Comfort}

ISO DIS 7730 recommends a summer temperature range of $72^{\circ} \mathrm{F}$ to $78^{\circ} \mathrm{F}$ with a humidity range of $30 \%$ to $70 \%$ for comfort. ASHRAE makes a similar recommendation (see Figure 3-3). Summer conditions in the North often exceed both the temperature and humidity levels for comfort, whereas warmer regions in the South and Southwest may find these conditions prevalent. While the increased ventilation from open windows can compensate, the effect is very limited to a range of a few degrees expansion of the range. This does not account for the radiant heat gain which is not reflected in temperature levels. It seems clear that air conditioning is as important to maintaining crew comfort as is heating since there is no other way to provide the desired cab conditions at high temperature or humidity conditions.

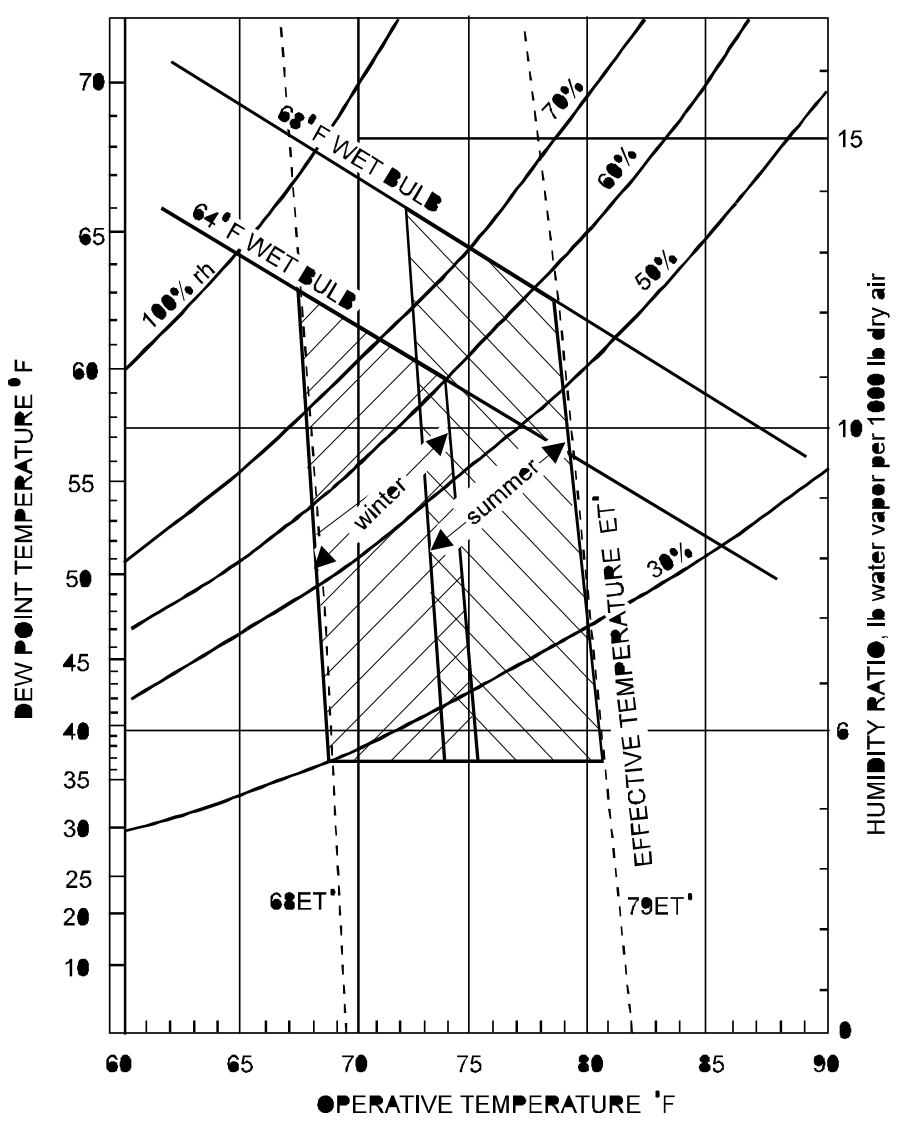

Figure 3-3. ASHRAE Standard for Thermal Comfort (@1995 American Society of Heating, Refrigerating and Air-Conditioning Engineers, Inc., Atlanta, GA. Used by permission from BSR/ASHRAE 55A-1995, Addendum to ANSI/ASHRAE Standard 55-1992.)

While the referenced standards give a justified upper limit of $78^{\circ} \mathrm{F}$ for comfort, operational circumstances may dictate higher temperatures. People do acclimate to higher temperatures which 
Benefits

\subsection{NOISE}

\subsubsection{Operational Issues}

expands the acceptable limits. Also, the differential between cab and exterior conditions should not be extreme if people will need to transition between them. Under less extreme conditions, the system performance should have a commensurate improvement. Air conditioning will also lower humidity levels in the cab, thus, lowering the effective temperature.

The system should circulate the conditioned air so that temperatures from floor level to head level do not vary more than $10^{\circ} \mathrm{F}$. However, the system should not discharge directly on cab occupants to prevent chills from drafts. Use of visors or tint shades on windows will allow the crew to lower radiant heat gain and lower the thermal load, even without air conditioning.

Air conditioning can provide benefits beyond just comfort and possibly improve crew performance. With air conditioning, the windows can remain closed which will improve inside noise levels and reduce the intake of dust and other contaminants. Lower levels of foreign matter will improve cab cleanliness and reduce equipment contamination which will reduce maintenance. To the degree that eye injuries are caused by objects entering by the windows, there should be a reduction in these injuries. A reduction in absenteeism caused by unwillingness to tolerate high discomfort levels is another possible benefit.

People generalize feelings about a prominent perceived characteristic to other related aspects of their environment. For instance (for more detail see the Seating section), Roach and Rockwell (1980) found that heat discomfort was the single factor, of the fifteen used, that best predicted the level of seat comfort or discomfort. "Heat dissatisfaction" apparently increases dissatisfaction with other cab features. Consequently, air conditioning may bring about an improvement in the perceived comfort of other areas of locomotive operation.

Noise is one of the most intrusive aspects of locomotive operations. The largest source is the engines; the noise levels and spectrum vary with speed and engine load.

Measurements of engine noise taken by Jankovich (1972) and Aurelius (1971) show that noise level is strongest below the 100 $\mathrm{Hz}$ range. The $\mathrm{dBA}$ sound measurement scale filters out lower 
frequencies and consequently underrepresents the true level of engine noise.

Additional sources of noise are interior brake air venting in older cabs, the horn, and wheel/rail noise at cruising speeds. The latter sources are focused in the middle and high frequencies and tend to be intermittent which makes them more annoying, but possibly less damaging to hearing. Open windows, especially in reflective areas like tunnels and between structures, increase noise. Another noise source comes from vibrations which loosen cab components and cause them to resonate. Finally, the radio is often turned up to improve speech comprehension in the noisy environment. The bursts of speech and amplified static have to be attended to, which makes an important tool also a source of annoyance.

Maintenance also can impact noise. Engines in less than ideal condition will run rougher and noisier. Mountings wear and loosen which can create new vibrations or decrease vibration damping which can create or worsen noise. Also, worn engine components (e.g., bearings) will create noise.

Noise exposure levels given by 49 CFR 229.121 limit continuous noise to $90 \mathrm{dBA}$ for eight hours with lower time periods for higher noise levels. This regulation went into effect after August 31, 1980. Sound level measurements conducted about ten years before this by Jankovich (1972) and Aurelius (1971) indicated that noise levels in the locomotive cabs were very close to or above the $90 \mathrm{dBA}$ limit. Since locomotives from this period may still be in use, their compliance with 49 CFR 229.121 is not known, given the wear factor and the lack of cab noise level measurements under operating conditions. Rockwell and Kiger (1989) have a statement that one railroad specifies noise limits of $82 \mathrm{dBA}$ for new locomotives. In contrast, Riley (1991) has many comments from engineers on the need/desire to reduce noise in the SD-60M, a recent design. Without measurements to confirm it, it may be that state-of-the art cabs have yet to achieve comfort levels (75 $\mathrm{dBA})$ in noise.

\subsubsection{Human Factors Considerations}




\section{Exposure Standards}

performance and increases fatigue. Noise produces a consistent, increasing, and statistically reliable fatigue effect, especially when experienced during a boring task (Michaut and McGaughey, 1972). Vigilance tasks and low stimulus environments which typify part of the engineer's job may be considered boring. Long duration exposure to loud noise damages hearing. Michaut and McGaughey (1972) state that a temporary lowering of hearing can occur after exposure to levels of $78 \mathrm{dBA}$ for as little as one hour. Locomotive cabs have much higher noise levels and exposures during work shifts are considerably longer. Noise also interferes with verbal communication and audible signals/warnings. With radio use, background noise decreases the signal-to-noise ratio and effectively degrades intelligibility. Cab noise is also in the background of radio transmissions initiated from the cab. Riley (1991) has documented comments from locomotive engineers on the need to improve radio intelligibility and speakers on the SD60M. This may indicate an acoustic signal-to-noise ratio which is too low. It is important to note that many experienced engineers have hearing losses, and this, combined with the high acoustic noise levels in the cab further degrade intelligibility.

The current $90 \mathrm{dBA}$ exposure limit (49 CFR 229.121) is well above other noise standards. The American Conference of Governmental Industrial Hygienists and the U.S. Air Force, in separate standards, have $85 \mathrm{dBA}$ limits for eight hours of exposure. The American Academy of Ophthalmology and Otolaryngology (1973), Poulton (1970), and Wittgens (cited in Jankovich, 1972) give $80 \mathrm{dBA}$ for eight hours as the upper limit to prevent hearing loss from long-term exposure. Wilde and Stinson state that at $90 \mathrm{dBA}, 11.9 \%$ of workers will have hearing loss after 20 years and $18 \%$ after 40 years. An 85 dBA limit will cause an expected loss of $5 \%$ and $8 \%$, respectively. Therefore, where possible, designers should have a much lower noise level as a goal.

Speech intelligibility considerations require even lower noise levels than pure health considerations. MIL-STD-1472D recommends a $75 \mathrm{dBA}$ limit for areas requiring occasional telephone use or direct communication at up to 5 feet. Jankovich (1972) states that Swedish Railroads set a design goal of $78 \mathrm{dBA}$ (not met by then current designs) because of the heavy reliance on voice communication. MIL-STD-1472D recommends a $65 \mathrm{dBA}$ limit for operational areas requiring frequent telephone use or direct communication at up to 5 feet.

Comfort is generally the most restrictive of the three factors. The 
argument for comfort is that it provides an optimum performance environment. Woodson (1993) gives $75 \mathrm{dBA}$ as the level for comfort and reduces fatigue.

\section{Drawbacks}

\subsubsection{Recommendations}

Noise Limits

Horn Location

Brake Venting
Railroad properties require engineers to report equipment problems, including weak horns, and to use torpedoes as a warning. Rockwell and Kiger (1989) include a survey comment from a manager that cab quieting may have gone too far. This person states that the sound reduction causes erroneous reports of weak horns and muffled torpedoes. These are drawbacks which result from reliance on noise isolation as the sole means of noise reduction. They can be resolved. For example, a VU meter and sound detection system mounted near the horn could give the engineer an objective measure of horn loudness. Alternate means to perform the function of torpedoes could be devised. An alternative effect of noise reduction suggested by an EMD locomotive designer warrants investigation. This is that the reduced level of background noise makes discrete changes like torpedoes and horns easier to detect but keeps them from being intrusive.

A goal of $80 \mathrm{dBA}$ is desirable from a health perspective. A noise level of $75 \mathrm{dBA}$ or below that would facilitate communication and maintain comfort should be the ultimate goal. An alternative to reducing noise levels is to require hearing protection. Use of ear protection with the current limit could complicate communications. An aviation style microphone headset system represents a possible solution.

The location of the horn(s) can impact interior noise, especially with open windows. The output should be directed away from the cab, located away from the cab, or shielded to produce a sound "shadow" in the cab area. However, locating the horn behind the cab to create a sound "shadow" may reduce the effectiveness of the noise to warn motorists. Closed windows reduce horn intrusion, so air conditioning can provide indirect benefits in this regard. In placing the horn on the locomotive, the needs of motorists and pedestrians should be considered as well as the needs of the locomotive crew.

Air brakes do not need to be vented within the cab. In addition to the noise, the air can carry brake system contaminants and stir up dust already within the cab. Engineers do use the sound as a cue 
Noise Reduction

Noise Cancellation

Isolated Cab

\subsection{TOILET FACILITIES}

\subsubsection{Operational Issues}

to indicate brake performance, so some audible indication of brake function may be desirable.

In older locomotives, passive measures of insulation, isolation, and absorption were used to lower cab noise levels. Their effectiveness can degrade with time. For example, insulation can settle and compact or isolation bushings and fittings can wear and lose damping capability.

A relatively new technique to reduce noise is to actively cancel it. These systems characterize background noise to determine a pattern in its waveform. They then create a waveform that is opposite to try to create a zero pressure when the two are added. This process is especially effective with medium to low frequencies because the waveform changes more slowly than at high frequencies. This makes the pattern more predictable. Conversely, speech is extremely dynamic and unpredictable by past patterns so it is less affected by this technique. The short, sharp pattern of the torpedo also should not be attenuated by this system. Another benefit of these active systems is the automatic gain control which would increase amplitude in response to the noise increase when the windows are open.

Noise cancellation systems can be relatively inexpensive and small, making installation costs for older cabs about the same as new cabs. The low cost and high potential benefit makes this technology an attractive option that should be explored. It would complement current noise reduction methods and could bring significant improvement to a high priority concern.

Isolated cabs use special mounts to prevent the frame from transmitting vibrations into the cab enclosure. This method complements current noise insulation. Insulation is less effective in damping low frequencies and the isolation provides a barrier to low frequency transfer. The limiting of vibrations can also reduce the sympathetic vibration of loose components in the cab which can produce internal noises.

Toilet facilities are an example of the evolution of crew comfort 
concerns. They have gone from being absent to standard equipment which improves with new cab designs. For example, Riley (1991) indicates that 35 of 58 locomotive engineers $(60 \%)$ surveyed thought that the toilet facilities of the SD-60M were better than previous versions. However, there were many comments from locomotive engineers which indicated the need for specific improvements. Apparently, the toilet is often designed to fit the space available rather than designing in the space to accommodate the toilet facility. Complaints that may substantiate this include those of cramped space and lack of heat or ventilation. The cold operating environment also limits design options compared to those available in more controlled conditions.

The hot, humid conditions that can exist in the toilet facilities in warm weather are favorable for bacterial and fungal growth. This growth produces persistent odors which are a problem directly and indirectly as they are associated with a lack of cleanliness. Chemicals used in some toilet designs also add odor. Odors represent the most urgent problem with cab toilet facilities. Some toilet designs recycle water from the holding tank to flush. This exposes the tank contents to the air in concentrations that increase with use and time and releases stronger odors.

The small volume of the typical facility and a lack of, or insufficient ventilation allows odors to build to offensive levels. Even in newer facilities that have ventilation, the ventilation rate is low and the vent arrangement may create dead areas. For example, a lavatory in a modern design locomotive had an inlet vent about three feet above the floor and the outlet at floor level (see Figure 3-4). The upper area, where the user's nose is, is little affected by the air flow. This allows odors to build faster than they are removed. Cab air conditioning, like heat, is not directly provided in the toilet facilities.

Illumination in toilet facilities is often barely adequate. It is provided by a low voltage system that usually has a single dome light (upper left in Figure 3-4). While this may be appropriate for night use to prevent loss of night vision, it may be too weak for day use. When the cab is brightly lit by the sun, the change when entering the lavatory may cause problems seeing in the low light level. Dim light also strengthens a poor impression of the facility. The light is mounted in the ceiling, generally over the toilet. This places it close to the eye when standing causes the iris to close, and makes the lower area seem darker still. 
Lack of maintenance is another common comment concerning toilet facilities. Service use of locomotives does constrain availability for maintenance which can extend time between cleaning. While this may play a role resulting in holding tanks that are not pumped often enough or general untidiness, there are also comments that the crews sometimes add to the problem. Since there are no trash receptacles in cabs, crews may use the toilet (or the lavatory in general) as a place to throw trash.

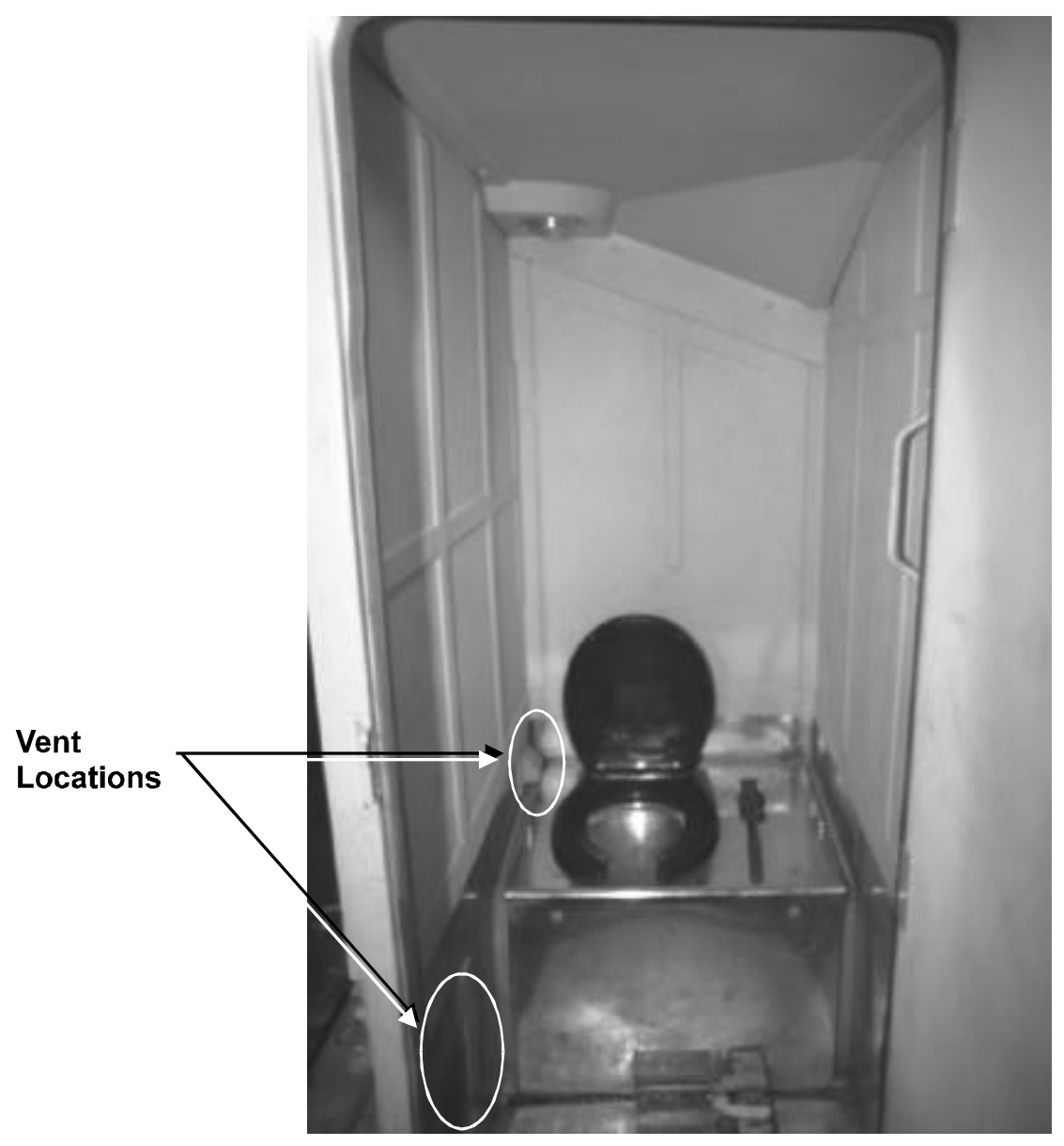

Figure 3-4. Vent Locations of Locomotive Lavatory

Locomotive cabs can be exposed to subzero temperatures for prolonged periods. This will result in freezing problems with water waste management solutions. This constrains toilet design options. State environmental laws limit how railroads can dispose of effluent. These laws can restrict solutions. 


\subsubsection{Human Factors Considerations}

Size

Odor
Woodson (1990) notes that lavatories should not cramp the user. The ceiling height should accommodate the 95th male percentile engineer plus any headgear he may wear. Especially when located in the short hood, current toilet facilities are not very tall or overly spacious. Consideration of the clothing worn during use, which can be bulky, requires more space in locomotives. Riley (1991) cites crew comments that the toilet on the SD-60M, a recent design, is cramped.

Bacteria can generate ammonia and methane gasses, both of which can be smelled in low concentrations. These odors are perceived as mildly unpleasant to highly distasteful and are generally associated with unsatisfactory sanitary conditions. Moreover, heat and humidity create conditions in which bacteria can generate significant amounts of gas in just a few hours. This is too fast for cleaning alone to practically remove or prevent. Fungi, like mildew, can also thrive in moist areas wherever organic material is present (e.g., drains and walls) and the result is an additional source of odor.

The small volume of cab toilet facilities allows odor concentrations to build rapidly. Ventilation can remove this buildup, but the volume of air movement and inlet/outlet placement impact effectiveness. If the ventilation is inadequate, odors will be present and a perception of uncleanliness can be created.

The design of the toilet design must block odors that form in the waste tank from entering the lavatory. Odors can enter the lavatory if there is no physical barrier or the seal is poor. In conventional toilets, water both cleans the toilet and forms a seal against odors from the waste pipe. However, this design presents problems in the temperature extremes that locomotives operate in which force design compromises. Dry toilets, an alternative, can permit solid residues to remain in the bowl. This provides a medium for bacteria and a visually distasteful sight. These design

features can require cleaning after each use to prevent odors and other signs of "neglect."

A modular self-contained unit that can be removed from the lavatory and replaced with a clean one is another possible approach. The used units can be cleaned later by in-house 
or contract services and stocked at terminals for reuse. These toilets are commercially available for boats. Modifications of the current lavatory design may be needed to permit quick and easy exchange at yards. A benefit of this approach is that used units could be removed and replaced with a fresh unit more often than lavatories are currently serviced.

Odors may escape into the cab when the lavatory door is opened or if the unvented lavatory door seals are poor. The lavatory location in a well in the short hood creates a pocket which may be under-ventilated and creates a problem in the cab with odor concentration. Under this circumstance, the odor becomes a cab problem rather than just a toilet problem.

Abuse by Crews

Temperature

\subsubsection{Recommendations}

As mentioned in the preceding section, it is easy for conditions of "dirtiness" to develop. There is a concept in the security realm known as "the first broken window." If a window is broken in a vacant building and left unrepaired, it becomes a tacit signal that vandalism is permitted. The situation will rapidly degrade because any addition is merely a change in degree. This applies to all types of unwanted activity: graffiti, littering, etc. This may also apply to cab crews, in that if they perceive the toilet facility as already dirty, a little more mess will not cause a new problem. Results may range from failure to clean an accidental mess to intentional acts.

There are two other practical factors that may result in crews not keeping the toilet area clean. The low light level may play a role by reducing vision, and the lack of any other receptacle for trash in the cab leaves the toilet as the best option for trash disposal.

Toilet facilities are not directly equipped with environmental controls, like heat and air conditioning, which are installed in the cab. If ventilated with outside air, the facility temperature and humidity depends on the outside conditions. The lack of heat is worsened by the need to partially undress. Despite the shorter use time, the lavatory comfort requirements for heat or air conditioning are the same as those for the cab. Air conditioning may provide some additional odor reduction by reducing the heat and humidity that favors bacterial growth. 
General

Size

\section{Odor Reduction}

The following features will improve the comfort level and provide a visual impression of cleanliness.

- The interior should be light in color to make it appear larger and better lighted. Two levels of lighting, a single fixture for use at night (to preserve night vision) and additional fixtures for use during the day can provide a way to brighten the facility (and project a cleaner impression). A two-stage switch or dual switches can allow the user to select preferred level prior to entry.

- The lavatory (including a sink) should be provided with direct heat and air conditioning, if provided for the cab. Use of ambient cab air (i.e., as ventilation inlet air) to provide heat or air conditioning is suitable for moderate conditions only and cannot overcome outside temperature extremes.

There are several other features that will improve comfort.

- There should be no sharp edges, corners or protrusions that could cause impact injuries.

- Noise insulation should be provided.

- Handholds should be installed inside the lavatory to facilitate movement and provide support during sharp train motions.

- Steps leading down to the lavatory should not be too steep, causing balance problems or contorted movements to enter the compartment.

- The lavatory should have the same ceiling height as the cab with enough room to move and turn while wearing bulky clothes without being hindered by the walls. The 95th percentile male should set the standard for sizing the location.

Several design aspects should be combined to reduce odor buildup.

- The holding tank or other waste collection area cannot be allowed to introduce odor back into the lavatory. Therefore, the use of recirculated water from the holding tank for flushing should be eliminated. Water in the toilet or pressure jets which wash the bowl can almost eliminate residue from 
remaining in the bowl, but clean liquids must be used to accomplish this.

- Use of a clean water barrier or a tight sealing flap to block entry of gases is also needed.

- The holding tanks should be located outside of the cab. The water should also contain a disinfectant to retard bacterial growth in the toilet and holding tank. Precautions or designs to avoid freezing are critical.

- Ventilation does not prevent odors, but removes them when present. To be most effective, the entire air volume should be changed continuously.

- Inlet and outlet placements are critical and should be located to eliminate dead areas (e.g., an inlet near the floor and an outlet at the ceiling).

The pocket formed by the short hood area can allow escaping odors to concentrate and disturb cab crews even when not using the facility. Ventilation and/or good door seals are important to prevent odors from entering the main cab. An alternative is to use leaky door seals as an inlet for the ventilation system, but this means that the cab must have a positive pressure relative to the toilet facility at all times to prevent odors from entering the cab. The door latch should be able to keep the door from flying open during severe train motion.

\section{Cleaning}

- Toilet facilities should be designed (e.g., avoid sharp inside corners that create pockets, floor drain) and made of materials (e.g., nonporous) to permit easy cleaning and disinfecting.

- A trash receptacle should be placed in the cab to reduce the use of the toilet as a disposal by the cab crews.

- Self-cleaning toilet facilities have been developed for public use in cities in Europe and are being considered for use in U.S. cities. These units have covers which automatically move over paper holders and other areas that need protection and then flush the toilet and spray the entire enclosure with cleaning solution from several nozzles after each use. However, these units are not maintenance free. The cleaning solution supply tank and waste water tanks must be tended. The extra mechanical complexity requires a level of maintenance that 
may be problematic in the rail environment. Also, the problem of refuse buildup from crews will not be corrected (and a plugged drain may cause floods) nor will intrinsic odorgenerating problems of the toilet be overcome by these units.

A variation on the self-cleaning unit mentioned above that eliminates the need for on-board cleaning solutions and some mechanical complexity may help. This would have nozzles placed in the unit and connected to an external supply fitting. The fitting could be connected to a hot water line at yards to allow crews to spray the inside quickly when they feel it is needed, without much effort. This approach would simplify cleaning and can address the intermittent servicing.

\subsection{VIBRATION}

\subsubsection{Operational Issues}

Vibration and mechanical shock are inescapable in most vehicles. Locomotives produce vibrations and mechanical shocks from four major sources, the engines, the trucks, irregularities in the track, and train slack movement. Vibrations occur on the vertical axis, the forward and lateral axes, and in rotation about these three axes. Slight imbalances in reciprocating engines, rotating drive shafts, motors, and generators create vibrations which are transferred to the vehicle. Truck gears can create vibrations, particularly during dynamic braking. Track which is not perfectly level vertically and laterally creates vehicle body accelerations that results in vibration. Engine loading and vehicle speed cause the vibrations to vary in intensity. Engine maintenance and wear can also effect vibration. Shunting and slack run-ins and run-outs are common sources of mechanical shock.

Locomotive design can reduce vibration. Engines have mounts that reduce transmission to the vehicle body. The trucks have passive suspension (shocks) to dampen vertical motion, but motions in horizontal and rotational directions and from truck mounted motors are reduced less. Isolating cabs is a fairly recent method to reduce vibration. This prevents the transmission of vibrations from the frame from passing to the cab structure. Wear and the loosening of fasteners cause all of these passive reduction methods to lose effectiveness over time. Active measures, which create opposing movement to cancel vibrations, represent a new

development in vibration reduction methods that are under current consideration. 
Very short duration or individual accelerations or shocks are confined to a sharp crest event. These are created by relatively fast changes in track state, or buff draft levels. The train speed control skills of the engineer and the makeup of the train have the most impact on crests felt by cab occupants. While both sharp crest events and vibration affect ride quality, the former are less controllable by locomotive design.

The seat is a primary means by which vibrations are transferred to cab occupants, although vibrating controls or surfaces can affect hands and arms locally. Seat padding can reduce the transmission or the effect of vibration. Due to resonance effects, the cushioning of the seat can sometimes amplify vehicle vibrations and worsen their effects (Wilde and Stinson, 1980; Oborne and Clarke, 1974). The compression properties of seat cushions, the amount of compression, thickness, and breakdown from use and age all impact the seat's response to vibration and these factors can vary greatly by seat design. Hence, the seat and its cushioning cannot be considered the sole solution to cab vibration.

Earlier research (Gamst,1975; Jankovich, 1972) indicated greater concern with vibration in the locomotive cab than more recent surveys. Rockwell and Kiger (1989) found a relatively low priority given to improvements in vibration control, and the FRA'S informal discussions with the BLE did not elicit mention of vibration as a problem. It is not known whether this is due to improvements in vibration levels or a shift in concern to other matters which are perceived as more critical.

\subsubsection{Human Factors Considerations}

\section{Body Effects}

Jankovich (1972) and Wilde and Stinson (1980) have extensive discussions of vibration and its effects based on a large number of reference citations. Vibration has two known direct impacts: health and comfort. The effect of vibration depends on its frequency and intensity.

The resonance pattern of the body depends on vibration frequency. The body is most sensitive to vertical vibration in the 2 to $20 \mathrm{~Hz}$ range, especially the 4 to $10 \mathrm{~Hz}$ range. Up to $2 \mathrm{~Hz}$, the body acts at a single mass. As frequency increases above this, various organs and body parts have individual resonances which cause them to be more affected than the rest of the body. For example, the arms, abdominal contents, and legs (when seated) resonate at 
about $4.5 \mathrm{~Hz}$ and the head resonates in the 20 to $30 \mathrm{~Hz}$ range. The relative motions of body parts caused by vibration are discomforting in themselves and cause a person to tense muscles to combat the vibration. This muscle tension causes muscle fatigue and possible overuse conditions after as little as ten minutes.

Direction of vibration and posture also make a difference. For instance, the rigidity of the spine reduces relative body part motion in the horizontal plane, but lowers the resonant frequency. This can allow the horizontal motion to occur in different phases, such as the head moving forward while the hips move backward at one instant and visa versa the next, and cause use of muscular effort to add more rigidity. However, in the case of vertical vibration, the compressibility of the spinal disks allows stretching and compression of the spine which provides some damping but causes wear. Posture changes the body's response to vibration. Sitting erect gives the body its highest resonant frequency and also provides the least natural damping force. It also removes the damping capacity of the legs and transmits vibration of all directions directly to the trunk from the seat surface. Standing allows the legs to dampen vertical vibration, but muscle fatigue prevents standing for long periods.

The intensity of vibration affects how well it will be tolerated. The levels required for health effects have not been identified yet, but there are guidelines for comfort. The intensity levels vary with the vibration frequency. The latest ISO standard employs a weighting factor for each frequency and eliminates the time weighting that was used to determine comfort levels. Vibrations above threshold levels are considered uncomfortable no matter how short or long the exposure. Detailed guidelines for evaluating the effect of vibration and mechanical shock can be found in ISO Document 2631 "Mechanical Vibration and Shock - Evaluation of Human Exposure to whole body vibration Part 1 General Requirements." The document presents detailed guidance on vibration measurement, vibration evaluation, health, comfort and perception, and motion sickness. In addition to this base document, there are a number of specialized parts to ISO 2631 in preparation which have special relevance to railroad equipment design. These include "Guidelines for the Evaluation of the Effects of Vibration, and Rotational Motion on Passenger and Crew Comfort of Fixed Guideway Transport Systems," and "Mechanical Vibration Laboratory method for evaluating vehicle seat vibrationapplication to railway vehicles." 


\section{Health Impacts}

\section{Comfort}

Vibration levels do have an impact on comfort. Frequency and intensity (measured in acceleration or displacement) are the critical factors. As mentioned above, body vibration is perceived as more unpleasant as the intensity of the vibration increases above a certain threshold. People typically tense muscles to dampen the vibration and/or assume a posture that reduces the vibration effects (e.g., stand or slump while seated). Over time during a work shift, this results in muscle fatigue which adds to intrinsic vibration discomfort. When experienced over a period of days or weeks, the fatigue can become a more persistent ache or worse.

Factors including acoustic noise level, visual stimuli, temperature, and humidity interact with vibration in the passenger's perception of comfort. The effect of these non-motion factors must be 
considered when using the results of vehicle motion tests to assess comfort. As an example, acoustic noise has been found to be correlated with vibration based ride comfort judgments in passenger rail cars.

The frequency range of motions in conventional rail vehicles expected to significantly impact comfort includes 0.1 to $2 \mathrm{~Hz}$ on curve transitions (roll), 0.4 to $10 \mathrm{~Hz}$ in the lateral and longitudinal directions; 0.4 to $20 \mathrm{~Hz}$ in the vertical direction. ISO 2631 provides guidance in terms of weighting curves. With regard to vertical motion, ISO 2631-1 provides guidance through two weighting curves $\mathrm{W}_{\mathrm{k}}$ and $\mathrm{W}_{\mathrm{b}}$. The $\mathrm{W}_{\mathrm{k}}$ curve is specified vibration in any system including buildings, ships, aircraft, or surface transportation vehicles. $\mathrm{W}_{\mathrm{b}}$ is a curve used by many railroads in Europe and Great Britain to evaluate the effects of vertical vibration on rail passenger comfort. Depictions of these curves can be found in Figure 3-5.

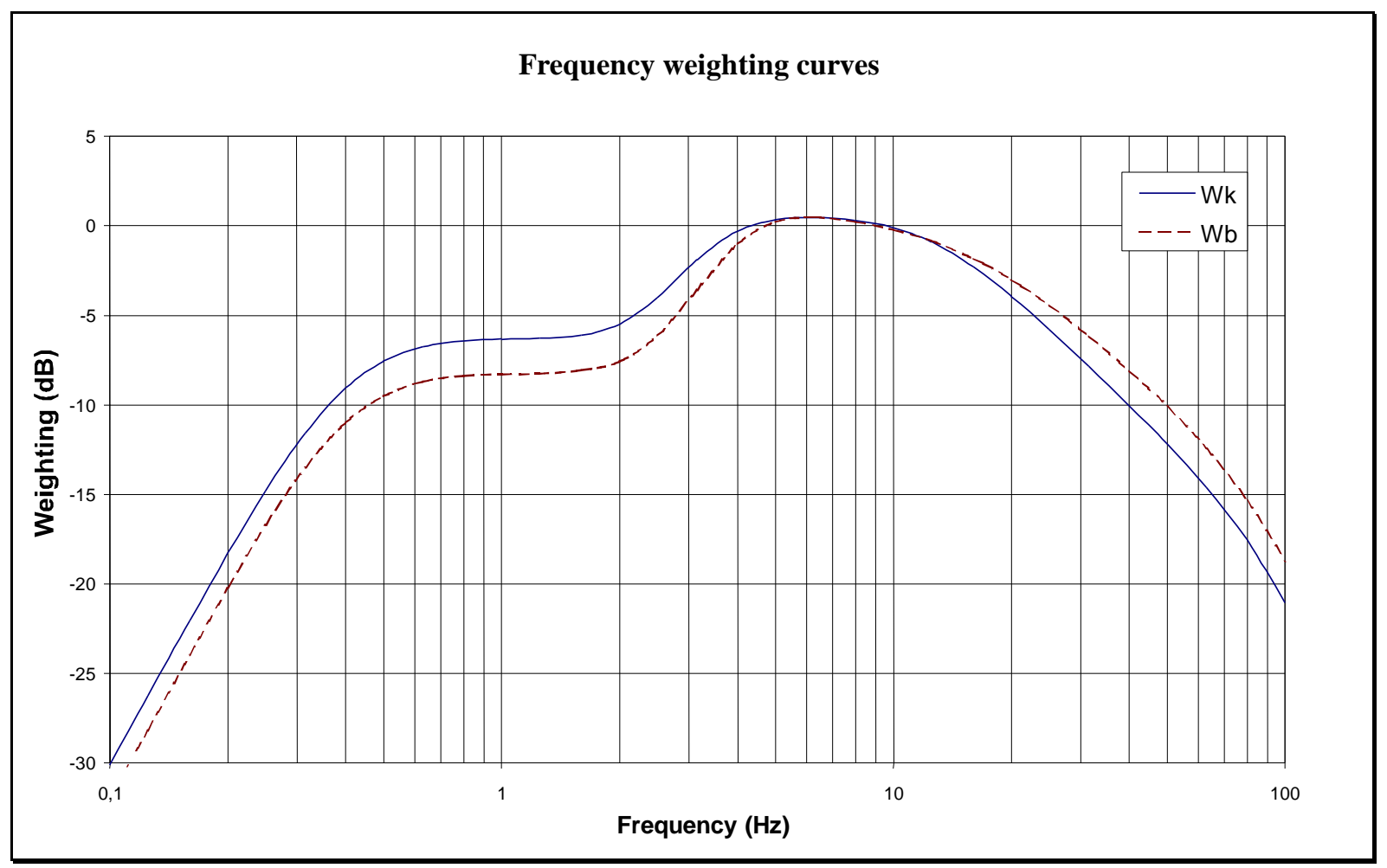

Figure 3-5. Vibration Weighting Values

Proficiency
Visual acuity and certain visual tracking tasks can be directly affected by vibration. Relative motion differences cause the retina 
image to move more than the brain can compensate, and this results in blurring. The effect varies with frequency, amplitude, direction of vibration, viewing distance, viewing angle, object size, nature of the visual task, and illumination. So it is difficult to predict results for specific situations. The large visual component of the engineer's task and the introduction of electronic displays does make this a concern.

\title{
3.6.3 Recommendations
}

\author{
Measurement
}

Manufacturers currently measure cab vibrations as a quality control step. However, this does not reflect operational conditions or human factors concerns. EMD and GE do static vibration tests but only down to $10 \mathrm{~Hz}$. This testing does not address human factors concerns because it does not measure the range of greatest human sensitivity and it does not measure the motions resulting from over the road operations.

Riley et al. (1991) describe the vibration levels in the SD-60M. Increased vibration occurred when moving and when engine loading increased. A shift in frequency also accompanied these intensity changes. Therefore, current measurement does not seem to reflect operational vibration conditions. With static acceptance limits near uncomfortable ride levels, the subjective descriptions of increases during motion from Riley could indicate that ride quality levels are being exceeded during operation. Riley also received comments from crews that cab vibrations are a source of fatigue.

Measurements of operational vibrations on all three axes which include the 1 to $10 \mathrm{~Hz}$ range are needed to determine real vibration conditions in the cab. Once these levels are known, the need and potential benefit of more extensive (and expensive) control can be set. Anecdotal evidence seems to indicate that improvements are needed.

New guidelines that have been developed (ISO DIS 2631-1, 1994) do not use exposure time to set different limits for comfort and do not specify fatigue decreasing the proficiency threshold. These new guidelines have been ratified and should be the basis for deciding acceptable levels.

ISO 2631-1 weights measured acceleration levels based on their frequency and uses uniform thresholds to judge comfort level. Figure 3-5 is a graphic representation of the weightings used for vertical acceleration. The $\mathrm{W}_{\mathrm{b}}$ curve is commonly used by 
European and British Railroads to weigh vertical vibrations. Other countries such as Japan use the more general $\mathrm{W}_{\mathrm{k}}$ curve. All countries use the $\mathrm{W}_{\mathrm{d}}$ curve (not illustrated) for horizontal vibrations. The measured acceleration values should be multiplied by the corresponding weighting factors (left axis) given for that frequency. The resulting weighted levels can then be

compared to the thresholds to determine comfort. Annex B gives the following as guidance on thresholds for comfort:

Less than $0.315 \mathrm{~m} / \mathrm{s} 2$
0.315 to $0.63 \mathrm{~m} / \mathrm{s} 2$
0.5 to $1 \mathrm{~m} / \mathrm{s} 2$
0.8 to $1.6 \mathrm{~m} / \mathrm{s} 2$
1.25 to $2.5 \mathrm{~m} / \mathrm{s} 2$
Greater than $2 \mathrm{~m} / \mathrm{s} 2$

$$
\begin{aligned}
& \text { not uncomfortable } \\
& \text { a little uncomfortable } \\
& \text { fairly uncomfortable } \\
& \text { uncomfortable } \\
& \text { very uncomfortable } \\
& \text { extremely uncomfortable }
\end{aligned}
$$

\section{Isolation}

Passive isolation is a basic method to reduce vibration.

Vehicle/wheel suspension systems are a prime example. The main benefit of passive isolation is reduction in amplitude, but a downward shift in frequency can also occur. The isolated cab provides supplementary benefits to vibration control through the vehicle suspension. Vehicle suspension only affects vibration from the track and the wheels. An isolated cab affects all vibrations from the frame, including those generated by the engines. A reduction in cab noise will occur too.

Jankovich (1972) describes two different seat post vibration dampers used by the German and Swiss Railroads which isolate the seat from the frame. While cheaper than cab isolation, a difference in relative vibration between the engineer and the controls and displays can cause problems in some conditions, especially in more severe vibration, when the seat damper provides the most comfort benefit. Both of these isolation methods need to be tested to determine the benefit they provide.

\section{Active Control}

The next level of effectively reducing vibration is active control. Instead of using energy dissipation for reduction, this method uses opposing force to cancel vibration. Active suspension has been discussed to improve rail passenger comfort. It would potentially do the same for cab crews. Jankovich also describes an active seat post-isolation system designed for aviation use. Since passive cab isolation is a "new" option, active cab isolation is not yet being considered, but it could be a possibility. Active systems involve extra cost and may require more maintenance resources. 
Human Factors Guidelines for Locomotive Cabs

However, until an accurate assessment of the impact of cab vibration on health and safety is made, the benefits delivered by active systems cannot be judged. 


\section{CHAPTER 4. CAB LAYOUT}

\subsection{GENERAL DESIGN CONSIDERATIONS}

\subsubsection{Operational Issues}

Each of the following sections focuses on a particular subsystem or component of the locomotive cab. This section sets the stage for the following sections by discussing the human factors considerations that may affect the system as a whole as well as issues that may affect several components or subsystems in the locomotive cab. This includes: general considerations, layout and maintenance.

This section discusses broad industry issues that affect the overall design of locomotive cabs. These trends include the incremental pace of change, backward compatibility of designs, interaction between customer and supplier, effect of design on maintenance, bi-directional operations, and range of environmental conditions.

\section{Incremental pace of change}

Successive generations of locomotive cab designs have been developed in incremental steps. A design may undergo only minor changes for decades. For example, the control stand based cab has existed in variations since at least the 1940s. The greatest change that occurred in this design may have been the adoption of the clean cab design in the 1970s. Recently, the control stand has given way to the desktop console, but most other aspects of the cab remain basically the same (with definite incremental improvements). The long service life of locomotives (20-40 years) makes compatibility with previous designs an important issue since a railroad may own locomotives with cab configurations, from different design "eras." The desire for backward compatibility of designs has been a driving force in the design of current locomotives.

\section{Backward compatibility}

The incorporation of backward compatibility in the design of locomotive cabs has had both positive and negative human factors implications. The incorporation of new features while retaining old features helps the engineer to learn the new system more quickly.

However, requirements for backward compatibility have also resulted in the interface, itself, driving the kinds of changes that 
can take place, rather than the user requirements. The consequences of this process can be seen in the transition from the AAR control stand to the desktop workstation designs. The control movements of the throttle and dynamic brake developed for the AAR control stand were retained in the current desktop designs. In the AAR control stand design, the throttle was pulled to left to increase speed and the dynamic brake was pushed to the right to increase braking. In the desktop design, the controls went from being in a vertical plane to the horizontal plane. This results in the engineer pulling the throttle toward him to increase speed and pushing the brake forward to increase braking. These control movements were developed in part to insure that an engineer who collapsed forward would be likely to decrease the throttle setting or apply the brakes. However, the motions needed to actuate these controls violate the principle that control levers should follow the direction of intended motion.

This change in orientation of the controls also changes the muscle groups needed to move the controls. However, the controls in the desktop console were designed to have the same degree of resistance as the controls on the AAR control stand, but the muscle groups needed to move the controls in desktop design were not well suited to provide these forces.

\section{Interaction between railroad and builder}

Standardization makes design and manufacturing easier for the builder. It also can reduce the likelihood of locomotive engineers' errors when transitioning from one cab to another. For some areas (e.g., noise limits), there are currently accepted standards that are based on practical limitation. Where these standards exist, they are cited. However, the report also cites goals which should be strived for and that are more supportive of the safety, health, productivity and comfort of the engineer.

Railroad properties can specify many individual modifications. These can be based on operating needs, union agreements, or preferences. This effect varies from requesting a different type of seat or toilet to unique placement of controls. Electronic displays, with software control of the type and nature of information display, offer the potential for greater diversity. Ergonomic considerations, including consideration of the anthropometry of the user population, are often out of the hands of the builder. It must put together the components to give the 
customers what they want, even if it deviates from the ideal.

\section{Bi-directional operations}

A key issue that will affect future locomotive cab designs is the need for bi-directional operations. While some railroads use it only rarely, others have operations that depend upon it. The AAR control stand design offers limited bi-directionality while desktop console designs assume operation in only one direction. How this requirement is achieved will have significant implications for the design and placement of the controls and displays.

A number of proposed designs are being considered by the Canadian National Railroad and the Association of American Railroads (Laporte and Jones, 1994) to address bi-directionality in future locomotive cabs. One proposal would place the controls in the arm rest of the engineers' chair and place duplicate sets of displays facing in both directions. To operate in either direction, the engineer would swivel the chair in the direction of travel. Another proposal would divide the locomotive into two units: one containing the cab controls and another generating the power. The locomotive cab would be isolated from the power unit and could be turned around to operate in either direction. This approach is modeled after slug-hump vehicles used in yard operations.

\section{Maintenance}

Each year, only a small number of new locomotives are purchased by the railroads. In 1992, there were 18,004 locomotives in service, of which 323 were new and 142 were rebuilt (AAR, 1993). This means the majority of locomotives are more than 14 years old; about $63 \%$ of the locomotives in use were built before 1980. Maintenance costs associated with normal servicing as well as repairing and replacing failed parts may exceed the initial cost of the locomotive over its lifetime.

A hidden cost is the effect on performance, safety, and working conditions when various subsystems and components within the $\mathrm{cab}$ are not adequately maintained. For example, a common complaint among engineers is that the air conditioning systems fail to operate. In hot weather, heat can contribute to crew fatigue and lead to a decrease in the engineer's ability to perform duties. 


\subsubsection{Human Factors Considerations}

\section{General}

Hazards should be designed out. The clean cab concept of avoiding protruding parts and sharp edges is a perfect example. Wilde and Stinson (1978) note the contribution that the floor makes to injuries. Elimination of tripping hazards created by level changes and a surface that provides traction even if soles are oily are considerations.

The reasonable comfort requirements of the cab crew need to be considered. The need for water, clothes storage, food storage, toilet facilities, and other conveniences provided in most situations are reasonable expectations. Aesthetic needs and cleanliness levels are other areas that need attention.

The railroad industry has a tradition of austere working conditions. With the rapid pace of change in home and workplace technology, newer employees may consider more modern accommodations as the norm. The human factors tenets of: 1) using traditional expectations, and 2) the introduction of changes to improve user "fit" requires tradeoffs that are not dictated by hard and fast rules. However, the long service life of locomotives means that decisions will impact crew comfort for a long time. This could tilt the tradeoff towards the more modern design.

The cab interior should be at least 76 inches high, although European designs use 79 inches (Jankovich, 1972). Standing operation and movement needs to be accommodated, so height of the users is the defining factor. Enough space should be allocated for each cab occupant. Sixty-five square feet is a minimum 
amount of floor space (Jankovich, 1972). Comfort facilities (toilet, water cooler, storage, refrigerator, cooktop, etc.) should be located out of the main area of the cab and not counted as crew space.

The toilet should be positioned so that light does not create glare or reflections for the engineer when the door is opened at night. Interior surface finish should be light colored, of low reflectance and easy to clean. The general cab light should be frosted to diffuse the light because its relatively low location places it just above eye level and can create glare. Changes in floor levels in the cab (e.g., raised seat platform [see Figure 4-1]) should be kept to a minimum to reduce tripping hazards. Auxiliary items, like a first aid kit, flares, torpedoes, and fire extinguisher, should be mounted where they are accessible, but do not impede movement in the cab. A trash receptacle should be provided to prevent unwanted littering.

Special operations can require extra equipment to accommodate engineer/operational needs. A separate control panel, oriented to the rear, will allow reverse movement. However, this will not compensate for the restricted visual field. A separate panel should be provided for remote operation of consist locomotives. It should be located in a secondary position near the control stand.

The arrangement of the workspace inside the locomotive cab is limited by a number of factors that include: track width, restrictions for height above top of rail, clearance around the locomotive, and crashworthiness standards. Within these limits, the designer is faced with the task of designing a space that enables the engineer to perform his or her tasks as safely and effectively as possible. 


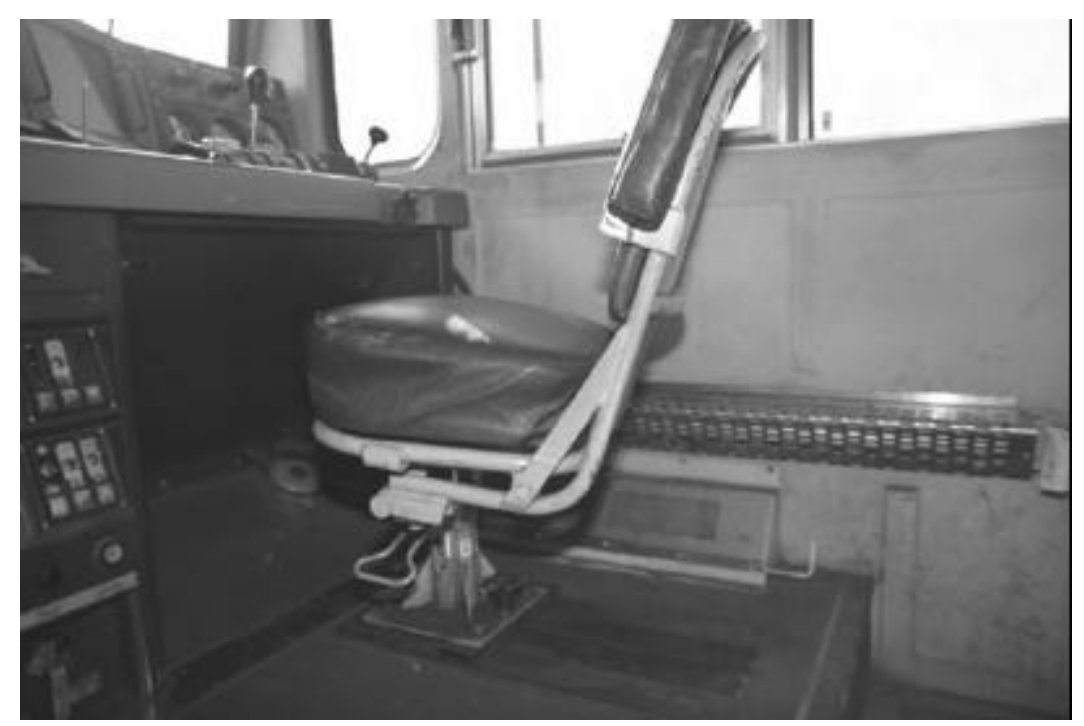

Figure 4-1. Raised Platform for Engineer's Seat

In determining how the equipment and facilities will be adapted to fit the engineer, the designer must specify where the equipment and furniture will be located and how they will relate to each other. Anthropometric data provides essential information about the body's dimensions that the designer can use to achieve this objective.

The current guidelines are based upon anthropometric data that is representative of the general population (Sanders and McCormick, 1994; Grandjean, 1988; Chaffin, 1987). The Canadian National Railroad is currently collecting anthropometric data from approximately 6,000 engineers that will provide more accurate information for designers to use in the locomotive cabs of the future.

In developing human factors guidelines for evaluating the locomotive cab, it is important to understand the needs of those who will operate and maintain the locomotive. While the primary concern is with the engineer engaged in mainline operations, there are other users whose needs must be considered. These people include the conductor, road and yard crews and maintenance personnel.

In deciding who to design for, the designer is faced with several different principles for applying anthropometric data (Sanders and McCormick, 1994). 
- Designing for extreme individuals is appropriate when a design feature should accommodate most of the population (i.e., a doorway). In practice, this often means specifying a minimum or maximum value of the characteristic in question that accommodates most, but not all of the population (i.e., the 95 th percentile for males and the 5 th percentile for females).

- Designing for an adjustable range is appropriate when features can be adjusted to the individuals who use them (i.e., seats, keyboards).

- Designing for the average individual is appropriate in noncritical situations, where designing for an extreme is inappropriate and where adjustability is impractical (i.e., a toilet seat). The designers' choice will depend upon the situation.

\section{Layout}

The layout of the workstation should be designed to give the engineer and the conductor good exterior cab visibility, the ability to monitor all displays and controls, place the controls within appropriate reaching distance, and provide adequate clearance to maneuver (Grandjean, 1988; Ivergard, 1989; Sanders and McCormick, 1994; Woodson, 1992).

Visibility. To provide good visibility while minimizing fatigue due to poor neck and head posture, regular viewing tasks should be within a 30-degree cone around the normal line of sight (Grandjean, 1988). The normal line of sight is 10-15 degrees below the horizontal plane. Figure 4-2 shows the normal line of sight and the range of easy viewing. Outside this cone, viewing a target will require head and neck movements. 


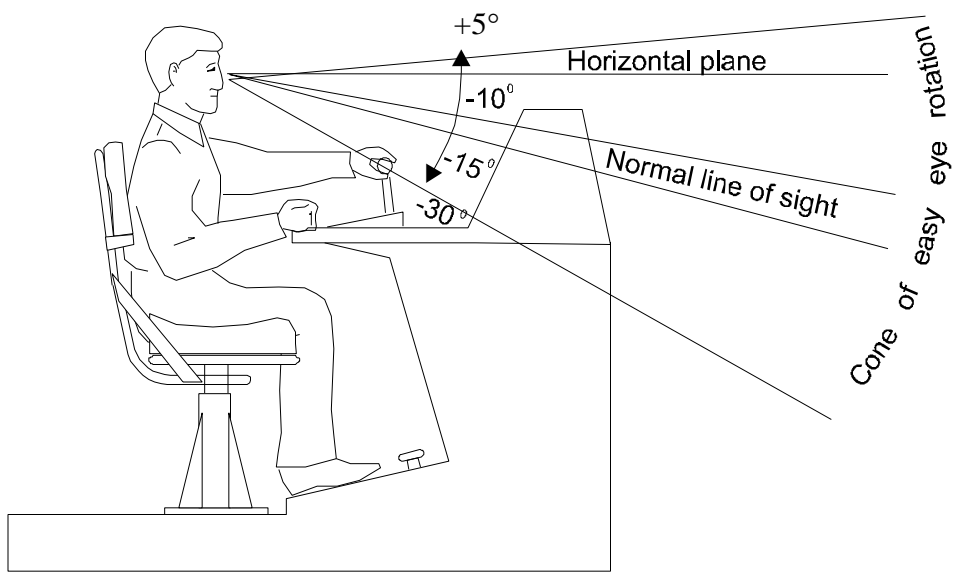

Figure 4-2. Area within which Primary Displays Should be Located

A display or visual target should be placed within a viewing angle between 5 degrees above the horizontal plane and 30 degrees below the horizontal plane (Grandjean, 1988). This recommendation should be followed in establishing the height of the seat in relationship to the windows and the visual displays in the cab.

Within this area, the visual displays and controls can be placed according to their level of importance (Ivergard, 1988). The primary displays and control should be placed so that the engineer may view them without changing eye or head position from the normal line of sight. Controls and displays of secondary importance may be located so that eye movements are necessary, but head movements are not. Less important or less frequently used displays and controls may be located outside the normal line of sight.

Arrangement of controls and displays. Several issues need to be addressed in deciding where to position the controls and displays on the work surfaces.

The designer needs to select a method by which controls and displays will be distributed on the work surface. Knowledge of tasks to be performed, anthropometric and biomechanical data, and environmental factors will help determine where to locate controls and displays. The following methods are commonly considered to determine position on the work surface: 
- Frequency of use. If one instrument is more important than another, then the more important instrument is placed nearest to the line of sight.

- Sequence of use. If several instruments are used in a specific sequence, that order is reflected on the work surface.

- Degree of importance. Where instruments vary in importance, the most important instruments are placed nearest the line of sight, and less important instruments are placed farther from the line of sight.

- Similarity of function. Instruments which perform the same function are grouped together.

To facilitate search and identification, controls and displays should be differentiated. In addition to grouping by similarity of function, other coding techniques such as color, shape, and size can be used to facilitate identification of controls and displays.

When there are many controls and displays to arrange in the workstation, consider the use of angled work surfaces (Sanders and McCormick, 1994). Placing controls on an angled surface allows the placement of a greater number of controls within easy reach. Visual displays are also easier to read when set at an angle.

Maximize compatibility between controls and displays. Designing the controls and displays to be consistent with the engineer's expectations will speed learning, reduce errors, and response time. Two types of compatibility are commonly considered: spatial compatibility and movement compatibility.

Spatial compatibility is concerned with the physical similarity and arrangement of controls and displays. Arrange controls and displays in corresponding patterns (Sanders and McCormick, 1994) where controls and displays show movement.

Movement compatibility is concerned with the movement of a control device and related movements shown on the display or its effect on system response. The movement of the control (e.g., left, right, up, down, clockwise, counterclockwise) should be consistent with the movement shown on the display or with system response. Thus, a control movement in one direction should produce a parallel movement on the display or have a 
parallel effect on system response.

Current locomotive designs have control-display conflicts for which there is no simple solution. When operating in the short hood forward position, the engineer accelerates the locomotive by pulling the throttle backward. While this control movement is opposite the direction of motion, it is consistent with the engineers' expectations. Engineers are accustomed to the throttle operating opposite the direction of motion. If this is to be changed, significant relearning will be required and errors can be expected during the transition.

Control size and spacing should permit the engineer to operate the controls without accidentally activating neighboring controls. If gloves are used, they should be considered when determining the minimal distances between controls.

Work-space envelope. Just as there is an optimal area within which the engineer can see the controls and displays, so there is an optimal area within which the engineer can reach and grasp controls. An evaluation of the cab layout must consider the work-space envelope within which the engineer's arms and legs will operate. Reaching too far to operate controls contributes to fatigue and increases the risk of pain and injury to the back, shoulders, and legs (Grandjean, 1988). The workspace must also allow sufficient space to move through a tight space or work within a confined area.

In considering how much space the engineer can effectively use, two areas are commonly used, normal and maximum (Sanders and McCormick, 1994). The normal area represents the horizontal surface area that can be conveniently reached with the sweep of the forearm while the upper arm hangs in a natural position. The maximum area is the area that can be reached by extending the arm from the shoulder. Figure 4-3 illustrates the normal and maximum areas for the 5th percentile. Figure 4-4 shows recommended vertical placement for different classes of controls. 


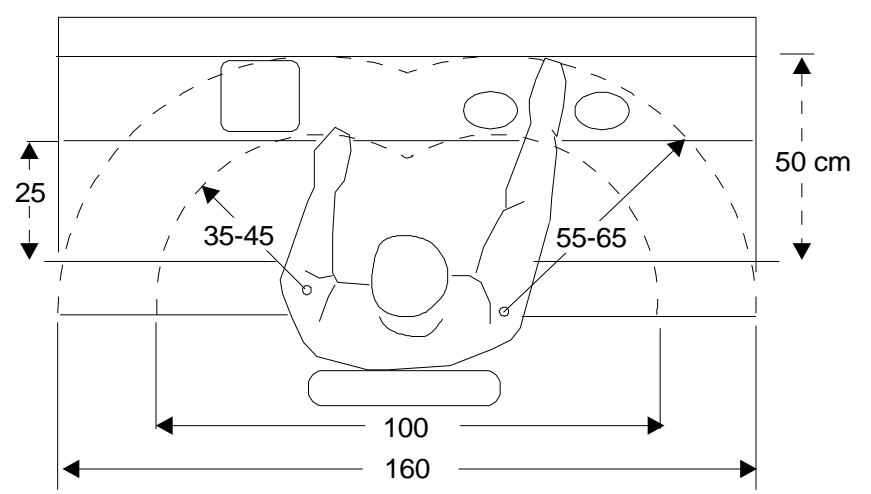

Figure 4-3. Area of Normal and Maximum Reach (Etinne Grandjean, Fitting the Task to the Man: A Textbook of Occupational Ergonomics, 4th edition, p. 51, 1988. Used with permission from Taylor \& Francis, Inc., Washington, DC.)
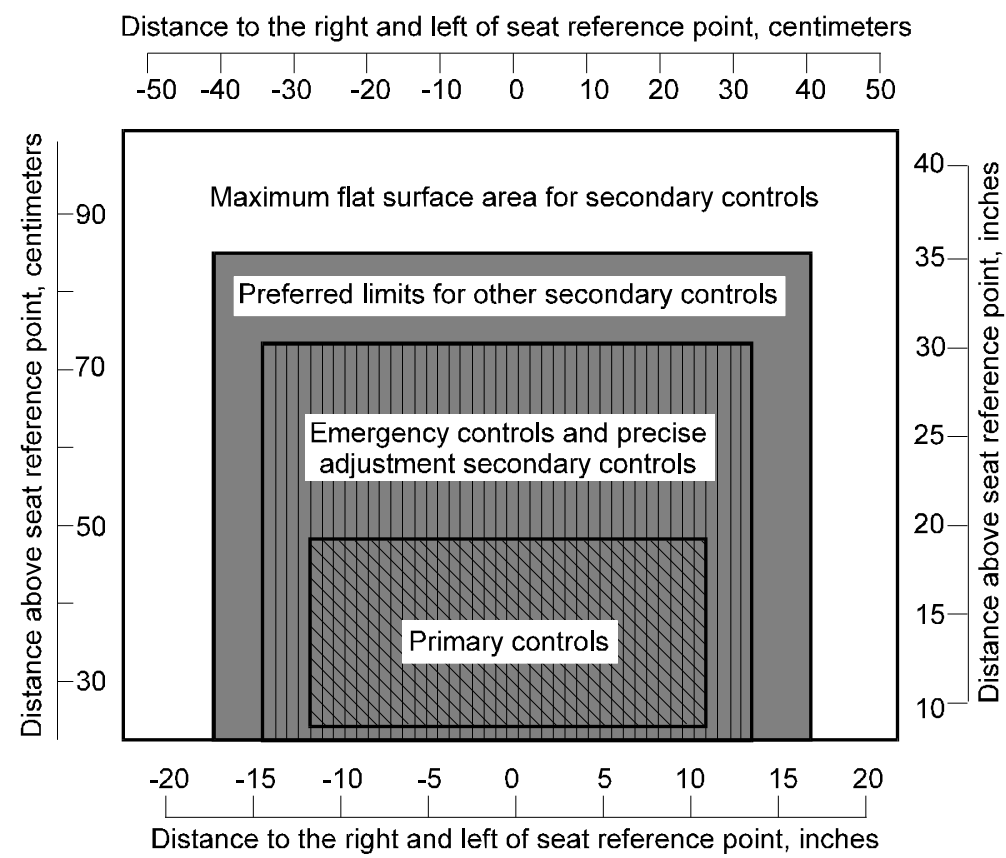

Figure 4-4. Recommended Heights for Four Classes of Controls (Adapted from Human Factors in Engineering Design, Sanders and McCormick, 1994, p. 471. Used with permission from McGraw-Hill, Inc., New York, NY.)

The following guidelines should be considered when evaluating 
the work-space envelope (Chaffin, 1987, Grandjean, 1988, Sanders and McCormick, 1994):

- The hands should not have to reach frequently or be held for sustained periods above the shoulder.

- Use padded forearm supports to relieve pressure at the shoulder and elbow.

- Design the workstation so that the engineer's elbows remain flexed (bent). A position in which the engineer's elbows are at a 90-degree angle require less effort than when the elbow is in an extended position. This goal may be achieved by designing the work surface to be at the same height as the elbow height.

- Provide sufficient clearance for the engineer's thighs under the work surface.

- Provide an adjustable work-surface height to fit individual physical dimensions and preferences. Grandjean (1988) recommends an adjustable range of $60-70 \mathrm{~cm}$. The need for a low work surface may conflict with clearance levels needed to provide enough knee space.

- Consider providing a workspace that allows both sitting and standing. Sitting and standing stress different muscle groups so that changing positions relaxes one group while stressing others. This may relieve muscle fatigue given the long hours the engineer frequently works.

The reach requirements vary with several variables that include the type of control, kind of clothing worn, and whether the individual is restrained. The type of control will affect the reach requirement due to the part of the body that operates the control. Toggle and push button controls that are activated by finger tip will have a longer reach than a knob or dial operated by a thumb or finger. Control levers that are operated with grasping actions of the entire hand will have even shorter reach limitations. The type of clothing worn may affect reach requirements by restricting the engineer's freedom of movement.

Maintenance

Maintainability is an important concern for the cab and the maintenance crews. Easy maintenance will help keep the locomotive operating properly. Some maintenance must be done en route by the engineer to correct running problems, but major 
repairs are done by maintenance crews. If a repair is not critical, the locomotive may go out without the repair and the cab crew will have to put up with the loss of function (e.g., air conditioning). A repair may be done partially or incorrectly, which can also impact the cab crew. For example, some brake systems that normally do not vent into the cab can be incorrectly assembled after repair so that they work, but also vent into the cab. Designs should try to reduce these events.

When considering maintenance in the design process, the skill level of the person who will be doing the work needs to be assessed. The skill level of the engineer will be very different from the mechanic responsible for servicing or repairing the locomotive at the terminal. When breakdowns in equipment occur and the engineer has the opportunity and skill to repair the problem, the system should indicate the nature of the problem and the solution (i.e., reset circuit breaker).

Another aspect of maintainability is to make the repair process as simple as possible. This can result in faster repairs, better quality, and fewer errors. Consideration of access to parts is particularly important. If parts are hard to reach, cannot be seen, or require a contorted position, the repair will take longer and may be poorly done. An overly complicated repair can lead to similar results.

Cleaning is an important part of maintenance. Design of the cab needs to consider the ease of cleaning. Equipment should be easy to clean. The arrangement of equipment should not create spots that are hard to reach.

Equipment failures are inevitable. Failure modes need be to be assessed for impact. Design for graceful degradation is a desirable goal. For example, a warning light can use a dual filament bulb or two bulbs. If a filament or bulb burns out, the warning will still work, but at a noticeably lower brightness. The lower brightness will cue the engineer that replacement is needed.

The concept of fail-safe is paramount. Failure of a single system must not create a safety hazard. Equipment designers need to consider the outcomes of various failures and ensure that a loss of control does not occur. For example, a brake system failure should not result in the loss of all braking capability. 


\subsubsection{Recommendations}

The principles of maintainability include the following: proper labeling, accessibility in repair, removal, replacement, inspection and testing. The nature of equipment or subsystems will determine what kinds of actions are necessary. Listed below are some general guidelines for designing a system to support maintainability (Burgess, 1986):

\section{Labeling}

- Use words and abbreviations that are meaningful to the user.

- Labels should be designed to survive exposure to wear damage, the effects of fading and dirt, and normal operating conditions.

- Instructions should be easily seen.

- Wording of labels or instructions should be brief and concise.

- Labels should be located next to or on controls.

- Danger signs should use white lettering on a red background.

\section{Access}

- Workspace and access areas should allow adequate room for needed body motions such as crawling or kneeling.

- Make the most frequently failing and critical components most accessible.

- Access panels should be accessible with common tools.

\section{Repair, removal \& replacement}

- Consider modular design, and where cost effective, throwaway units to minimize the skill level needed to perform the work and impact on related sub-systems or components.

- Use self-adjusting mechanisms where possible.

- Use self-lubricating sealed assembly with throw-away replacements. 
- Make stored materials, assemblies and spare parts easily accessible to authorized staff.

- Clearly mark storage locations and parts.

- Make retaining or load-securing devices easily removable.

- Make parts to be removed frequently easily accessible.

- Enable use of common hand tools to repair or replace defective items where possible.

- Make misconnection of parts impossible through the use of different sized and shaped interconnections.

- Design gaskets and seals to be easily replaced without removing or disassembling other equipment.

- Use quick disconnect electrical devices where units are replaced frequently.

- Use hinges, latches or catches to reduce handling and storage of covers.

- Design covers to avoid holding dust and dirt.

- Design cables or wire runs without bends and locate them to prevent being stepped on.

- Color code cables and plugs with both ends tagged to prevent misconnection.

- Route wiring away from lines that carry combustibles to prevent sparking.

- Design items weighing more than 45 pounds for two-man operation.

- Minimize the number of special tools required. When they are required, make them captive to avoid being misplaced.

\section{Inspection and Testing}

- Provide quick and positive identification of malfunctions and components. 
- Minimize the need to use special test equipment.

- Design major assemblies to be completely inspected by means of removable housing.

- Make reservoirs, gauges, and meters visible without removing panes or other components.

- Provide visual access for maintenance in progress.

- Make seals and gaskets easily visible after installation.

\subsection{ACCESS}

\subsubsection{Operational Issues}

Outward swinging doors that allow entry to the front and rear of the freight locomotive are the norm. However, the ease of use differs considerably, in particular for those locomotive cabs with a nose door. Some designs have an open stair leading down to the nose door while others require a trap door to be opened to access the stairs. The need for a nose door is greatest for the brakeman who requires easy access to the track for switching, although it is also used to pass between multiple units. Rear doors differ in that some models exit to an engine room and others models exit to an external catwalk. Although there are advantages and disadvantages to both designs, doors that lead outside have advantages in accident situations that make them a better choice. The outside door allows a potential escape route from the locomotive before an accident and better access or egress after an accident.

Locomotives with front doors that lead directly outside (and rear doors in long hood forward movement) can be the source of drafts. These drafts lead cab crews to use impromptu gasketing methods in cold weather. Wilde and Stinson (1978) and Riley et al. (1991) note problems opening doors in the winter. The reason is that door bottoms are at the walkway level and get blocked by snow and ice accumulation. Wilde and Stinson also note that doors are the second largest source of injuries (21\%) in 1976 Canadian National Railways data and quote a 1972 FRA sponsored report by Kurtz that lists doors as the leading source $(29 \%)$.

Hands and arms are the body parts most injured by doors. Latches that pinch fingers, and the potential to strike somebody 
when opening the door were noted as safety concerns by

\subsubsection{Human Factors Considerations}

locomotive engineers (Riley et al., 1991).

Emergency Exit

Safety

\subsubsection{Recommendations}

\section{Latches}

\section{Door Design}

Leaving the cab before or after an accident should be as easy as possible. During emergencies, activities that require complex thought or intricate manipulation will add to stress. Outward opening doors leading directly outside and quick, easy-to-operate door latches are needed. The possibility of blocked or distorted doors after an accident requires alternate emergency exits. A $25 \mathrm{x}$ 25 inch roof hatch or side windows that open enough or pop out to provide a similar size opening are possibilities. These features can also provide access to rescue people if the cab crew is unable to evacuate on their own. Pop out windshields are discouraged by Wilde and Stinson because other conditions can cause accidental ejection.

Latches have moving parts that may result in reduced clearances when operated. If the hand is near these areas, it can get pinched and/or cut. Such trouble spots should be eliminated, if possible, or guards devised to keep the hand clear of the area. If the handle has considerable travel, it may cause the hand to strike another surface at the end of the travel path.

Door latches should be examined for potential pinch areas. Handles that turn should not swing over areas of increased height (especially if the handle end curves inward) or too close to protrusions or door jambs. Using the handle to pull the door closed should not bring the hand too close to the door jam even if fingers are not closed around the handle. Alternate placement of the thumb (i.e., with or opposing the fingers) on the handle should be considered when judging clearances. There should also be enough clearance to permit wearing gloves.

The bottom edge of the door should clear the walkway it opens over to reduce the obstruction by ice and snow. Wilde and Stinson suggest about a 7-inch clearance. This should take the form of a step up into the cab rather than a lip separating an equal level cab floor and walkway to reduce the possibility of tripping. Extending the roof about a foot over the rear doors to form a protective awning may also reduce snow and ice buildup by the door. The nose door could include a small sight glass to see if there is somebody that could be struck when the door is opened. 


\subsection{VISIBILITY}

\subsubsection{Operational Issues}

Engineers need to see signals which regulate their movement, obstructions on the tracks like work crews or vehicles at grade crossings, mile markers, landmarks used as control cues, track conditions, and switch positions in certain circumstances. The critical factor is the need to see things far enough ahead for train control actions to be effective.

The design of recent cabs is predicated on travel primarily with the short hood forward. In this configuration, the hood does limit the downward visual angle, which blocks close views of the track and the nose ladder. The visibility with this design seems only adequate for main line haul operation and may be less than adequate for switching. Operation of these cabs with the long hood forward or cabs designed for long hood forward presents some definite vision limitations. The window that is used for forward viewing is small and often mounted in a door may be further from the engineer (which further limits visual angles), and obstructed on one side by the locomotive body. Curves in the direction of the long hood side will result in limited visibility of the track ahead.

The glass area is not the only factor that affects vision. Equipment mounted on consoles or overhead (e.g., radio or speedometer) can block areas of the visual field. While the engineer may be able to lean or move to look around the obstruction, this is not desirable. The weather can also create vision restrictions, particularly snow buildup and condensation on the windshield. Here again, long hood forward operation is a disadvantage. The window is perpendicular to the direction of travel and vertical, so air flow will not remove snow accumulation from outside the wiped area (may even blow it back on the wiped area) and defrosting options are more limited for door-mounted windows. The sun can also limit forward visibility. At low angles, the sun will be in the engineer's eyes, possibly for some time if the track direction does not change. At even greater sun angles, a film of dirt and plastic outgassing residues on windows can scatter light and create serious glare. Since glare is not a point source, visors are not as effective in providing relief. 


\subsubsection{Human Factors Considerations}

Visual Needs
Robinson et al. (1976) devoted considerable effort in order to determine what engineers' visual field should be for line hauling. (See Figure 4-5.) They examined the locations and paths of signals and other objects in the visual field, the engineers' operational requirements, and the space needs for internal panels and displays. Fifty feet was set as the minimum close-in distance for viewing a track level or overhead object. This translated to a $40^{\circ}$ viewing angle above horizontal eye level and $25^{\circ}$ below.

However, the potential need for an overhead panel and to restrict sun problems lead them to reduce the overhead angle to $35^{\circ}$, which would make a bridge signal visible up to 55 feet away rather than 50 feet. They judged the benefits gained outweighed the loss of 5 feet. Lateral field-of-view should be at least $180^{\circ}$ and preferably $220^{\circ}$.

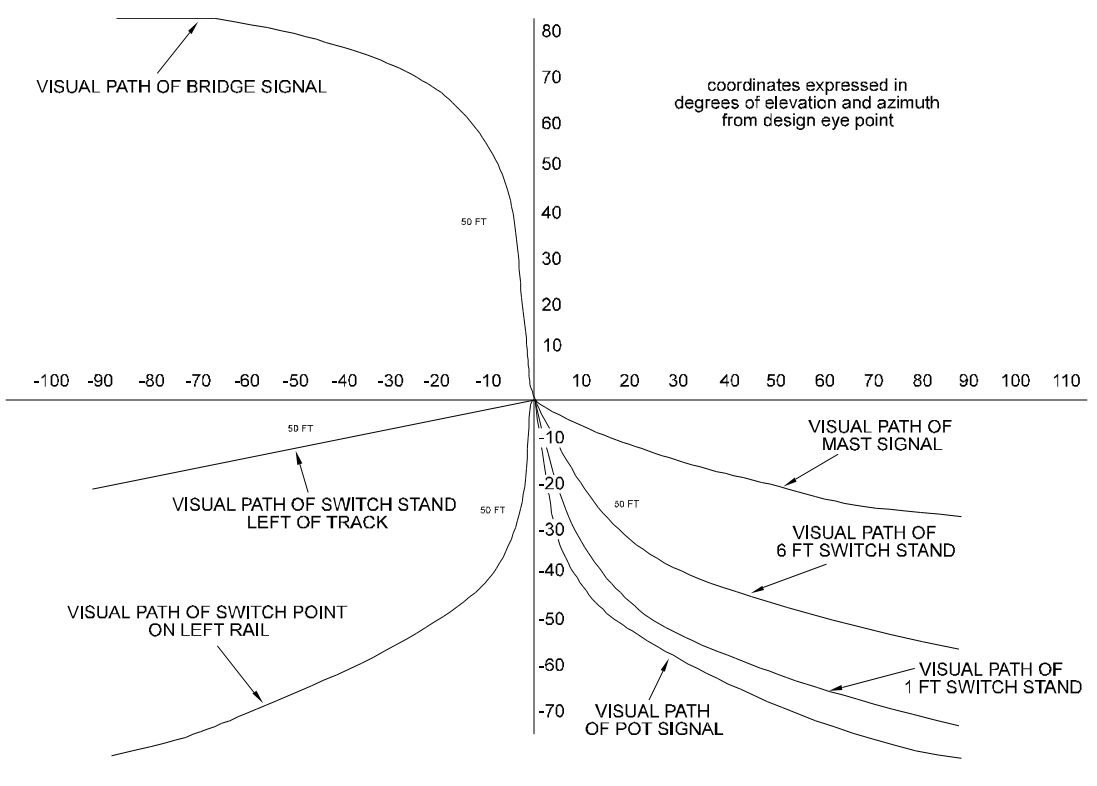

Figure 4-5. Locomotive Engineer Vision Requirements

Too much glass area, in an attempt to increase visibility, can be a problem. The cab's structural strength is reduced with more glass, lowering crash and object impact protection. The sun can interfere with vision over larger angles and the radiant heat gain and loss is increased, which hinders cab temperature control. The greater glass area creates the potential for more reflections and 


\subsubsection{Recommendations}

\author{
Sight Distances
}

\section{Vision Supplements}

glare from internal and external lights at night and in fog. Finally, Jankovich (1972) and Wilde and Stinson (1978) point out that at high speeds the track blurs if seen too close in, and this can be annoying. Wilde and Stinson also quote a test by British Rail of a single $114.3 \times 58.4 \mathrm{~cm}$ (about $46 \times 23$ inches) windshield that was well received by engineers. The important fact is that the engineer must see those things that are important for operation and increasing visual angles beyond that provides little benefit.

The windows should permit the operator to see a track level object as close as 50 feet away, and an overhead object (e.g., signal bridge) as close as 55 feet away. Lateral field-of-view should be at least $180^{\circ}$ and preferably $220^{\circ}$. This is based on long haul needs. For switching, closer downward vision is needed, but this capability could potentially be a liability in long haul. In starting a locomotive, an engineer often looks to the ground to see if the locomotive is moving. This is done through the side window. Long hood forward operation cannot meet these sight requirements.

If the windows are not large enough to get the visual information, the engineer will open the side window and lean out. This exposes the engineer to debris and inclement weather as well as fatigue. These drawbacks make window use an undesirable option.

Selective use of mirrors can also help to add visual area and to see around corners without requiring large body movements. The latter aspect can address a concern that the engineer cannot see the nose ladder area on modern cabs. This visual restriction creates a problem of locating the brakeman when he is on the nose platform during switching. Since this a detection task, the ability to detect presence on the nose is all that is needed. A convex mirror could provide this capability if located on the forward end of the nose.

Long hood forward operation and switching operations have visual needs that the modern long haul cab cannot easily accommodate. During these operations, critical portions of the engineer's field of view are obscured by the diesel engine cowl. Other transportation modes use instruments to provide guidance where vision is not adequate. One that may supply the engineer's visual needs is closed circuit television (CCTV). CCTV is used 
on some buses to provide rear vision and may serve a similar specialized use in locomotives. A camera to cover the blind area in long hood forward operations and/or one to cover close in track during switching may meet visual requirements. The system would need to be operated only during these specialized operations and would not be installed in locomotives for properties which do not intend to operate this way. Tests will be needed to determine if CCTV could meet engineers' needs and operate reliably in the railroad environment.

GM Hughes and Texas Instruments have jointly developed a video-based night vision system that they plan to sell to police departments for squad car use. Police tests began in 1993. Plans also include optional installation on luxury cars by the end of the century. Current cost estimates are $\$ 6,500$ with the potential for manufacturing improvements and sales volume to lower this. Ford has a radar-based system under development, but this system is more conducive to automated control. The radar image is not similar to a visual image and requires interpretation which may not identify unusual changes.

Recent night train accidents involved a damaged bridge and overhanging cargo on a passing train. In both cases, the engineer could not detect the problem visually before the collision. There are also the issues of bright lights affecting dark adaptation and the decrease in night vision with age. A night vision system may offer a solution. Again, testing is needed to determine the actual benefit. An infrared system could be augmented with a near infrared light which is not visible to the eye. This can be done with a filter over current headlights.

\subsection{SEATING}

\subsubsection{Operational Issues}

Engineers spend long hours in close contact with locomotive seats and are understandably greatly concerned with their comfort. Most cab characteristics that the engineer may perceive as unpleasant are somewhat transitory (e.g., too hot, too noisy); however, the seat is a constant. Seat acceptance is influenced by intrinsic seat characteristics and by factors that the operator associates with the seated position. For example, Roach and Rockwell (1980) found that a too high cab temperature was the single factor that most influenced seat discomfort. This is probably due to the heat and perspiration levels experienced at the seat contact area. Other non-seat factors that can affect seat acceptance include: leg room, knee room, availability of 
footrests, clearance from sidewall, vibration levels, ease of entry/exit, clearance when swiveling, visibility, and reach-tocontrol distance. In fact, Roach and Rockwell found that seat characteristics accounted for only $46 \%$ of the variability in the seat ratings for their survey. Therefore, any seat assessment has to be done in context with cab workstation design.

The seat type installed in locomotives depends on the property which buys (or owns, for replacements) the locomotive. Some are specified by the railroad management and others are specified by labor agreements. The long service life of locomotives means that older models with more austere seating are still in service. Thus, seats in service range from relatively advanced designs to a round seat pad that resembles a stool. Similarly, the seat adjustments available and the nature of the adjustment mechanism vary by railroad and locomotive type.

Operating a conventional locomotive is a relatively fixed position task that restricts the engineer's ability to move around or stretch at will. The modern console style cab puts even more limits on the engineer's ability to change position. The older control stand allowed standing operation and required twisting and leaning to perform some operations. This means that the engineer could move more, which prevents postural fatigue. The console allows (and requires) less postural variation. Those systems which still have a deadman pedal impose extra constant leg effort.

The justification for concern about seat comfort is provided by Roach and Rockwell's extensive survey. Nearly $90 \%$ of participating engineers had a criticism about their seats. About $80 \%$ reported discomfort after a typical run. Their complaints included high and low back pain, hip and buttock pain, neck pain, and leg aches. The long-term implications of these pains and the underlying physical changes that cause them needs consideration. Riley et al. (1991) obtained similar pain ratings for the SD-60M seat which indicates that the advanced design has not addressed basic seat issues.

\subsubsection{Human Factors Considerations}

Purpose
In most rail operations, the engineer must remain seated for extended periods of time. Sitting changes the weight-bearing surfaces, restricts spinal movement, flattens or distorts the normal curves, and immobilizes the pelvis. Seats must compensate for these changes. Weight has to be spread evenly to avoid pressure 
points and the muscular and skeletal interactions of the spine have to be addressed to avoid stresses. The seat must

also permit the worker to perform tasks from that position, so its relationship to the rest of the workplace is important.

Seat design has many aspects that come into play to satisfy the support and position requirements of the seated body. Roach and Rockwell (1980) investigated these in their survey with prototype seats and derived a specification that incorporated anthropometric needs and situational preferences which were tailored to locomotive conditions. Their resulting guideline has theoretical and empirical support.

Seats also have social status implications. The size, design, and materials have connotations of status which affect the perception of the seat and its user. A seat should meet or exceed a person's perception of what is appropriate for the situation. A seat that appears to be poorly designed or worn conveys a sense that the person occupying it or the job being performed is not important. The reverse is also true.

There is no perfect seat or universal definition of seat comfort. Roach and Rockwell tested four prototypes that used good human factors design practices. While they had better ratings than the seats in use, they drew criticism on many characteristics. Riley et al. (1991) mention that the SD-60M seat incorporated many Roach and Rockwell recommendations. Engineers generally rated the seat better than others, but far from perfect. Thus, no matter how good a design standard may be, there will always be room for improvement and criticism. Aspects like cushion softness are hard to measure meaningfully and subject to personal preference rather than objective standards.

\section{Seated Posture}

The human spine is intended to bear weight in a vertical direction. In proper alignment, the vertebrae transfer weight as a compressive force. Discs at joints between vertebrae cushion the vertical transfer. Sitting with hips too far forward and/or a slumped back disrupts alignment which creates uneven pressure on the affected discs, shifts the center of gravity, and adds a shearing component to the force being borne. Disc structure is not designed to resist sheering forces. The muscles and ligaments have to compensate for this which adds a fatigue component. With the accumulation of fatigue, the potential for acute and chronic injury grows because adaptive potential of the tired 
muscles and strained ligaments is lost. The seated position, and more so the slumped position, limits spinal motion which

provides the motive force for spinal fluids. These fluids carry nutrients and provide lubrication to the discs and other structures.

Fluids stagnate over long periods of sitting and the discs suffer deficits.

Serber (1990) quotes work by Nachenson and Elfstrom (1964) which calculates a stress of 495 pounds at affected discs when the seated torso leans forward at 40 degrees. This is near the rupture point and requires muscular compensation to avoid damage. These calculations are for a static seat and do not consider the vibrations and jerks present in the locomotive seat which make spinal stresses more dynamic. Riley et al. (1991) observed that engineers lean forward with a slight twist to the left when operating controls and sit back in the seat when monitoring movement. This leaning may be the source of the stress that causes the acute pains reported by engineers and a potential source of more chronic problems. Low back pain was the most common complaint (40.6\%) in the Roach and Rockwell study.

Serber (1990) discusses three seating designs that allow pelvic motion to compensate for the lumbar distortions of leaning forward. The most common is known as the continuous balance seat. The seat pan travels in a curved track that allows it to tilt the way an unconstrained pelvis would to maintain proper spinal alignment. It creates a rotation centered at about the fourth lumbar vertebra (just below the beltline), the natural seated hinge point. While this design may correct one remaining seating problem (restricted pelvis), it is not known if it can be made strong enough to be reliable in the locomotive environment and if the dynamic motion will be accepted by engineers. Placement of operating controls on chair arms is another alternative that may keep the engineer in the position assumed during monitoring times, but this may be too radical a change for engineers to accept.

\section{Seat Adjustments}

Adjustments allow the seat to accommodate people of different sizes in an otherwise fixed workspace, suit personal preferences, and permit changes of position to relieve fatigue. Potential seat adjustments include: height, seat back angle, seat pan tilt, fore-aft position, swivel, armrest height, and lumbar support. 
Presence of seat adjustments is not enough. The adjustment mechanisms have to be easy to use and reliable. Roach and Rockwell found that $42.1 \%$ of their survey group had trouble working the adjustments on the standard seats. Riley et al. (1991) provide a recent insight on this matter with their comment that the seats on the property they studied required two people to change seat height. This was due to the 65-pound effort to lift and support the seat in its new position while a pin is pulled and replaced in the support sleeve. Reliability of adjustment mechanisms is possibly a worse problem. A seat set towards one extreme that cannot be reset could pose real problems for an engineer who requires the other extreme. Failure of standard seat adjustment mechanisms in the $40 \%$ to $50 \%$ range (e.g., $47.3 \%$ slide, $46.4 \%$ back tilt) were cited by Roach and Rockwell. Adjustments that are too hard to work, or broken, reduce their use and negate their value.

Another failure problem area is mechanical wear that introduces looseness and adds unwanted motion. Wilde and Stinson (1978) describe a seat swivel design that wears and allows the seat to wobble. The wobble probably has more of an adverse impact than the benefit the swivel feature adds. The harshness of the locomotive environment makes adjustment failure modes more critical than for mechanisms designed for a more benign environment like an office.

\section{Alternative Designs}

The introduction of the computer, with its video screen and keyboard, to the office has sparked renewed interest in seating. New designs have been created to address problems and many of these differ from the usual upright design (e.g., forward leaning). While many of the same problems exist in the locomotive, there are different problems too. The vibration and jerk levels are very different from the office, the requirement to remain in a fixed seated posture is much longer, the need to anchor the seat limits adjustment options, and engineers need to lean and twist while seated. So, these new seat designs may bring some benefits, but create new problems for the engineer that the office worker may not experience. More analysis and testing is needed to determine if the engineer would benefit from radical seat designs. Another aspect to consider is the potential willingness of engineers to accept an unconventional seat.

The need for alternative seat designs may also be important if napping is allowed in the locomotive cab. The FRA is considering the use of napping as a method for minimizing fatigue 
from the long hours of service and the irregular schedules that locomotive crews follow. The seat design needed to accommodate both comfortable working and napping positions

may be very different from a design that accommodates only work related activities.

\subsubsection{Recommendations}

\section{Seat Design}

The following design guidelines were developed by the Roach and Rockwell study (1980). Since there is no source of anthropometric data for railroad workers, they used 1978 NASA data for the 95 th percentile male and the 50th percentile woman as the upper and lower size limits. These size ramifications were combined with analysis of engineer comments on characteristics of the different seats (standard and prototype) to devise a tailored set of characteristics. They do differ in some areas from some generic seat design guidelines that focus on the office environment.

Seat Characteristic

Features and Adjustments

Seat Pan Size

Seat Pan Width

Seat Pan Slope

Cushion Thickness

Back Height

Back Width

Armrest Height

\section{Recommendation}

Folding armrests, variable back tilt, sloping seat pan, fore-aft adjustment, variable height, swivel

16- to 18 -inch effective length for any position of the seat back

17 inches minimum at back, 20 inches minimum at front

1 to 3 degrees from horizontal, front edge higher

3 inches minimum for pan and back

21 to 25 inches

16 inches minimum at hips, 21 inches minimum at shoulders

7 to 8 inches from top of uncompressed seat to top of 
armrest

Armrest Width

Armrest Length

Armrest Padding

General Armrest

Seat Covering

Fore-aft Adjustment

Swivel

Seat Height

Seat Back Tilt
4 inches minimum

13 inches minimum

$1 / 2$ inch minimum inside and top

Adjustment to lower elbow end to tilt 115 degrees from horizontal, armrests parallel to seat pan and 19 to 22 inches between inside edges

Should not cause sliding or be easily torn or cracked, must permit breathing and water vapor exchange

Minimum of 4 inches fore and aft of center

At least 180-degree rotation from forward to rear facing, rotation towards center of cab

No more than 16 inches at lowest position and at least 19 inches at top position (measured at top of front edge), adjustment steps no larger than 1 inch

From 95 to 115 degrees from vertical in steps no larger than 5 degrees

These recommendations are a good start, but do omit some other features that seating literature mentions as beneficial. Adjustable lumbar support that extends across to the pelvic bone crests is widely recommended. This provides support and corrects posture. Moderate contouring of the seat cushion for the buttocks and the seat back for spinal curves evens pressure and provides support. It can also be a subtle deterrent to slouching because body contours will not match the seat contours in an improper posture. Lateral support on seat back or a curved seat 


\section{Adjustments}

\section{Seat Environment}

back to supplement side sway support reduces abdominal muscle effort. A continuous balance seatpan, armrest controls, or other means to relieve the lumbar stress that occurs when bending forward will address a large residual seating problem. Additional backward motion of the seat or other adjustment that makes room to permit standing operation would add to possible operating position options lost in the shift to consoles.

The ideal seat adjustment mechanism is strong, easy to use, reliable, and wear resistant. The harshness of the locomotive operations may make office type mechanism designs unsuitable. A survey of current designs for ease of use, reliability, and wear resistance should be done by the seat purchaser to identify suitability of current designs. Efforts are required to develop criteria to evaluate new designs, and determine where further design work is most needed.

The workspace that the seat is placed in has considerable impact on perception of the seat. Non-seat characteristics can have a direct or indirect impact on the seated position or use of the seat. These need to be considered along with the seat characteristics to determine seating comfort.

Non-seat factors that need to be considered include: leg room, knee room, availability of footrests, clearance from sidewall, vibration levels, ease of entry/exit, clearance when swiveling, visibility, and reach-to-control distance. The use of a deadman pedal as a vigilance device also has a bad impact on the seated position.

Leg and knee room and footrests have comfort and health implications. Little leg and/or knee room forces immobility; the resulting discomfort can be endured for a short time, but not for long periods typical of an engineer's shift. Health aspects come into play from the blood pooling that can occur from the lack of muscular movement. Cramps are a common result with the potential to develop phlebitis which can lead to clot formation. These clots can lodge in the leg veins and cause thrombosis or travel to the brain (stroke) or lungs (pulmonary embolism). Roach and Rockwell found that $41.8 \%$ of older engineers (60 and above) reported leg aches compared to $23.7 \%$ of younger engineers. This is the only complaint that was reported more frequently than by the younger group and is consistent with the loss of circulatory efficiency that occurs with age. Footrests 
allow a change of position and lift the legs which relieves seat pressure on thighs. Both of these effects improve circulation. This concern may become more critical as console style cabs replace control stand cabs. The console style cab is more restrictive on movement and position change than the control stand is.

Roach and Rockwell (1980) and Riley et al. (1991) document problems with clearances when engineers investigating swiveling, legroom, and closeness to the sidewall are asked about seat improvements. Therefore, these should be considered when evaluating seating. 
$\longrightarrow$ 


\section{CHAPTER 5. WORKSTATION DESIGN}

\subsection{GENERAL}

\subsubsection{Operational Issues}

\section{Design Issues}




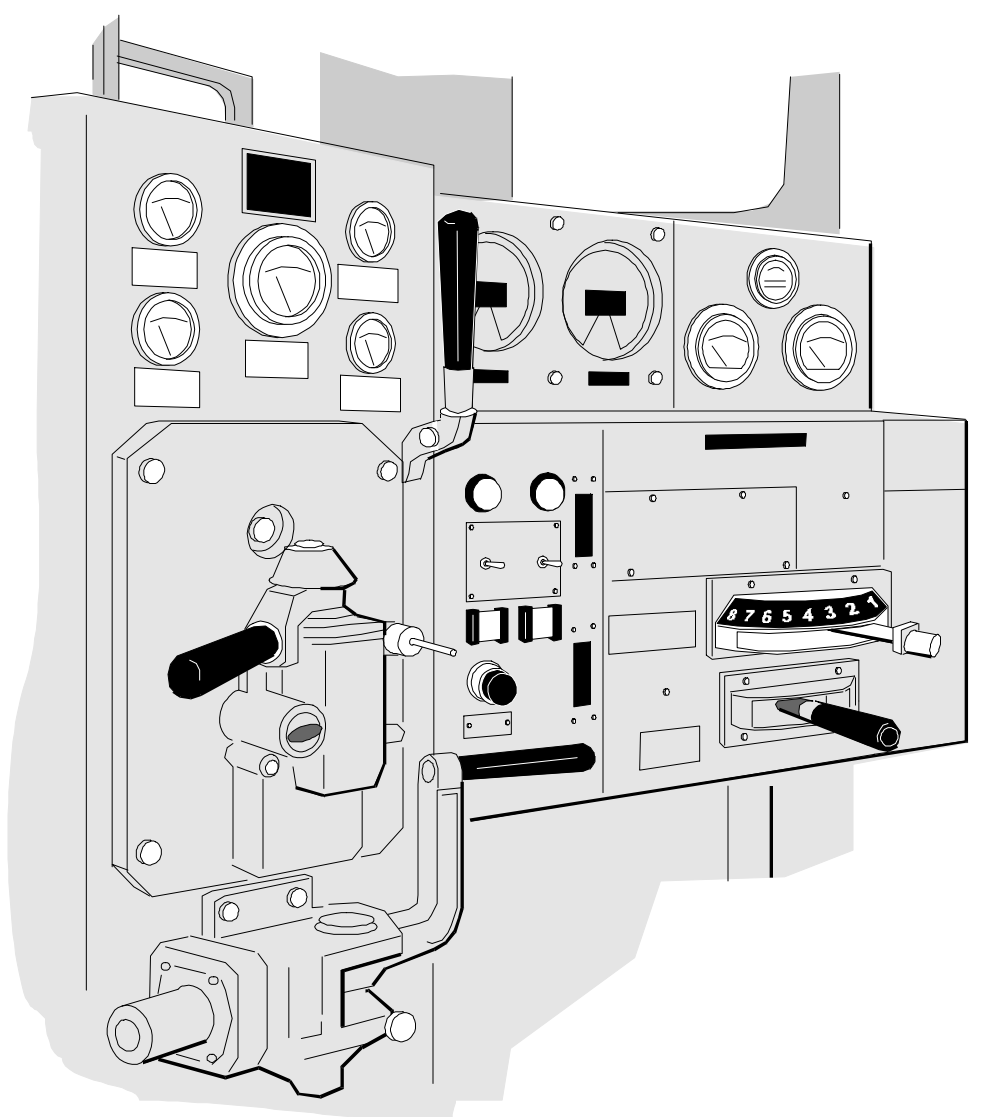

Figure 5-1. Older Style Cab Layout

With design improvements new problems emerge. For instance, some freight service engineers find now that they often have difficulty operating a train in reverse direction. They are forced into an uncomfortable body position in order to see where they are going while simultaneously operating the locomotive controls. With the older instrument arrangement, the engineer could operate the locomotive forwards and backwards, albeit with some body contortions.

\section{Workstation Designs in Use}

There are at least three different instrument arrangements currently in use or under consideration. They are the traditional cab stand, a desk-top like workstation, and the pedestal. The first two are the most common. The pedestal arrangement was proposed in 1978 as a 'cab of the future' (Ford, 1978). More recently, locomotive manufacturers have considered modifying 
the pedestal arrangement by placing controls in the arms of the engineer's chair. Each of these arrangements has strengths and weaknesses.

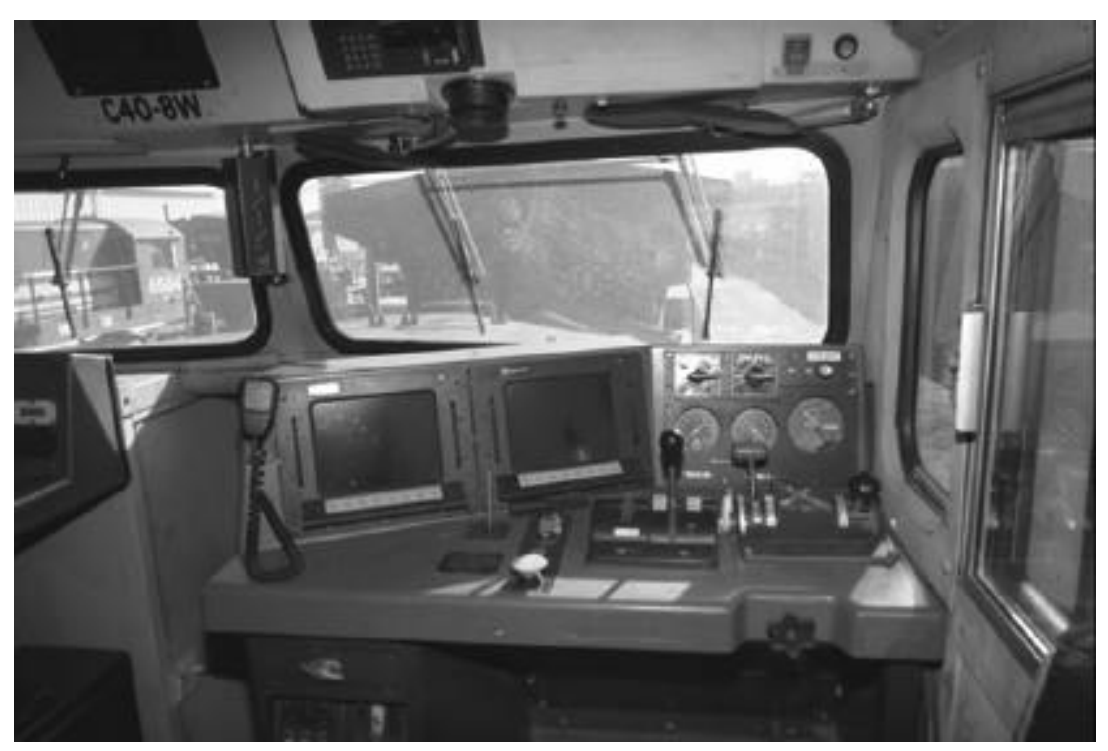

Figure 5-2. Modern Style Cab Layout

The traditional cab stand accommodates bi -directional operations. This arrangement allows the engineer to assume a position that allows visibility in either direction with just head movement. However, some engineers find it difficult to reach the controls when so positioned so they develop makeshift extensions of the control levers. Furthermore, when operating in the normal forward position, the engineer's body is constantly twisted in order to have good access to the controls and a view out the front.

The desk-top style workstation is a more ergonomically appropriate work area for main line operations in one direction. It is well suited for long periods of forward operation, provides the engineer with good forward visibility, and comfortable placement of controls. Even when operating in reverse, the engineer can still easily operate the brake controls. This design also provides a place for the engineer to keep log books and train orders. Some designs now incorporate recessed pockets for coffee mugs and sandwiches.

This design is not good for operations where there are long periods of reverse operations. One proposal is to provide the engineer with an auxiliary instrument panel. This panel would 
swing out from the side wall when needed for reverse operations. Only the necessary controls and displays would be available for operation in this mode.

A pedestal style of instrument arrangement would have the locomotive controls located in pedestals on either side of the engineer's chair, immediately in front of the seat's arm rests. Display panels used in this configuration are further away from the engineer as there must be sufficient room for ingress and egress from the chair. A variation of this arrangement is now being considered by the major manufacturers. In this design, the controls are installed into the arms of the engineer's chair. Such a design was not practical using the older mechanical brake controls, but is with the advent of newer electronic brake controls. A chair with such built -in controls might be the best way of combining the advantages of all recent arrangements.

Besides the design issues to overcome with this arrangement, some locomotive engineers may resist changes to locomotive design.

\subsubsection{Human Factors Considerations}

\author{
Controls and Display \\ Relationships
}

Controls are the interfaces the human operator uses to change the state of the machine he or she is operating. Displays are the interfaces that the operator uses to monitor the state or change of state of the machine. Thus, controls and displays work in unison to provide the engineer with the tools he or she needs to operate a machine. Good design has displays located as close as possible to the controls that affect them. The physical arrangement of displays should be similar to that of the controls.

When laying out the engineer's workspace, the designer should minimize potential safety hazards through good understanding of the capabilities of the user, the duties of the operator, the tasks to be performed, and the potential safety risks. A user-centered design usually begins by determining the observer's position and then arranging the instruments accordingly. In past locomotive cab design, just the opposite has occurred. The instruments are located first and then the engineer's seat was positioned. One manufacturer indicates that it follows this approach because of a concern for creating a crashworthiness envelope to protect the engineer. However, this tends to force the engineer to adapt to the locomotive rather than adapting the locomotive to the engineer. 
Work area design must also address access to the cab. An engineer should be able to get into and out of a properly adjusted chair without any adjustments. Particularly important is that normal egress should lead to or go through the crash protection envelope. Care should be taken that this pathway is not obstructed by controls nor conflicts with other crew or people in the cab.

\subsubsection{Recommendations}

When planning the arrangement of the instrument panels and the engineer's seat, there should be sufficient clearance between the instruments and the seat to allow easy access by engineers. Design clearance should be based on the 95th percentile of male locomotive engineers for the particular railroad.

SAE Standard J898, Control Locations for Off-road Work Machines, October 1987, shows in its Figures 2 and 3 (included here as Figures 5-3 and 5-4) the top view of the zones of comfort and reach for hands and feet relative to the Seat Index Point (SIP). The outer limit of the zone of comfort from the SIP is 22 inches for hands, while the outer limit for the zone of reach 33.5 inches. Its Figure 3 shows the same zones in a frontal view. In the vertical plane, the outer limit of the zone of comfort referenced from the SIP is from 3.94 inches below to 16.75 inches above for hands, while the outer limit for the zone of reach is from 9.84 inches below to 39.17 inches above.

All main controls, including their full range of motions, should be within the zones of comfort with auxiliary controls placed within the larger zones of reach.

Edges should be rounded, protective coverings used on levers, pinch points eliminated, shatter proof, glass used, switches recessed, and displays and controls well labeled. 


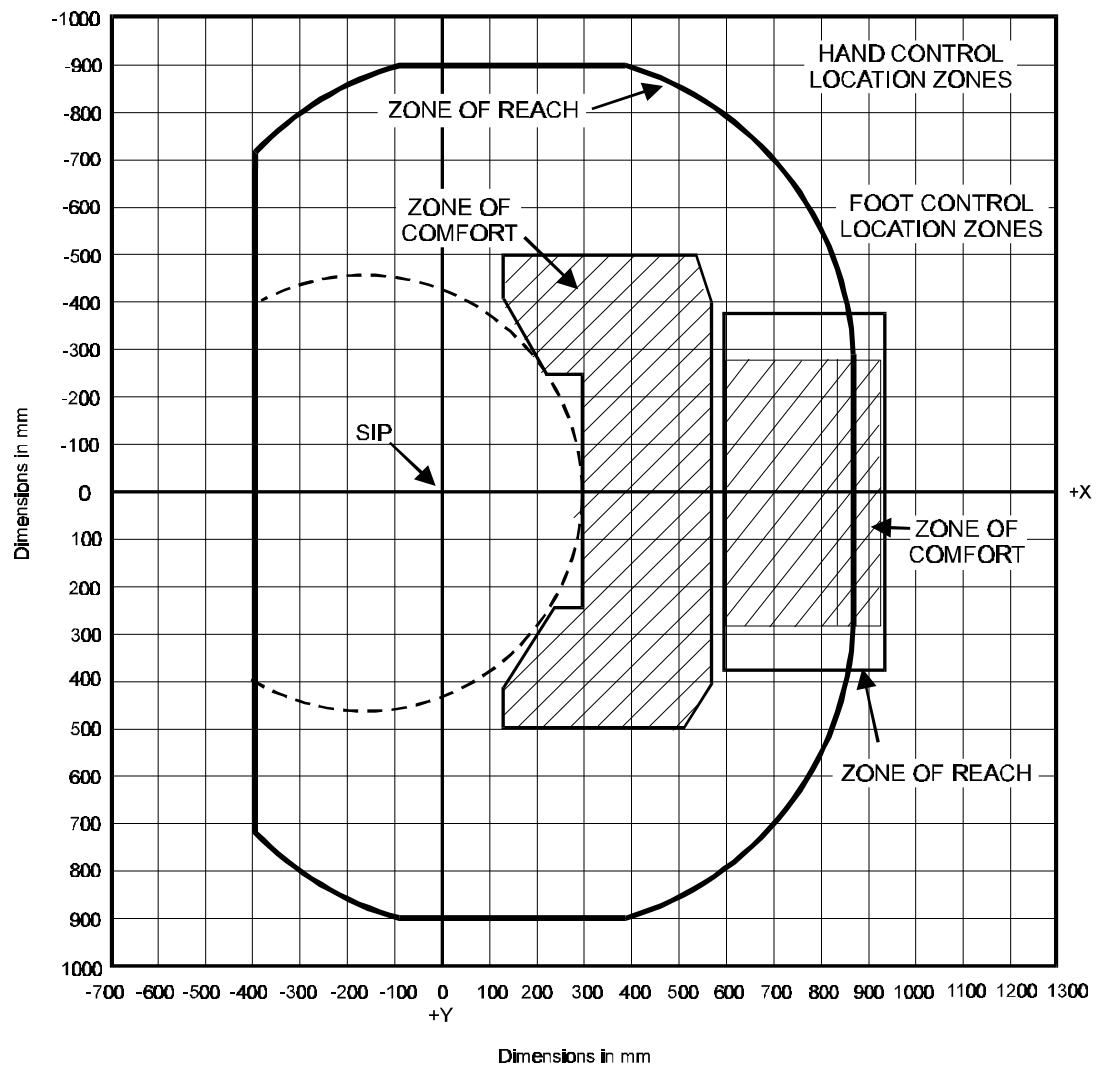

Figure 5-3. Top View - Zones of Comfort and Reach (Reprinted with permission SAE Standard J898@ 1987 Society of Automotive Engineers, Inc.) 


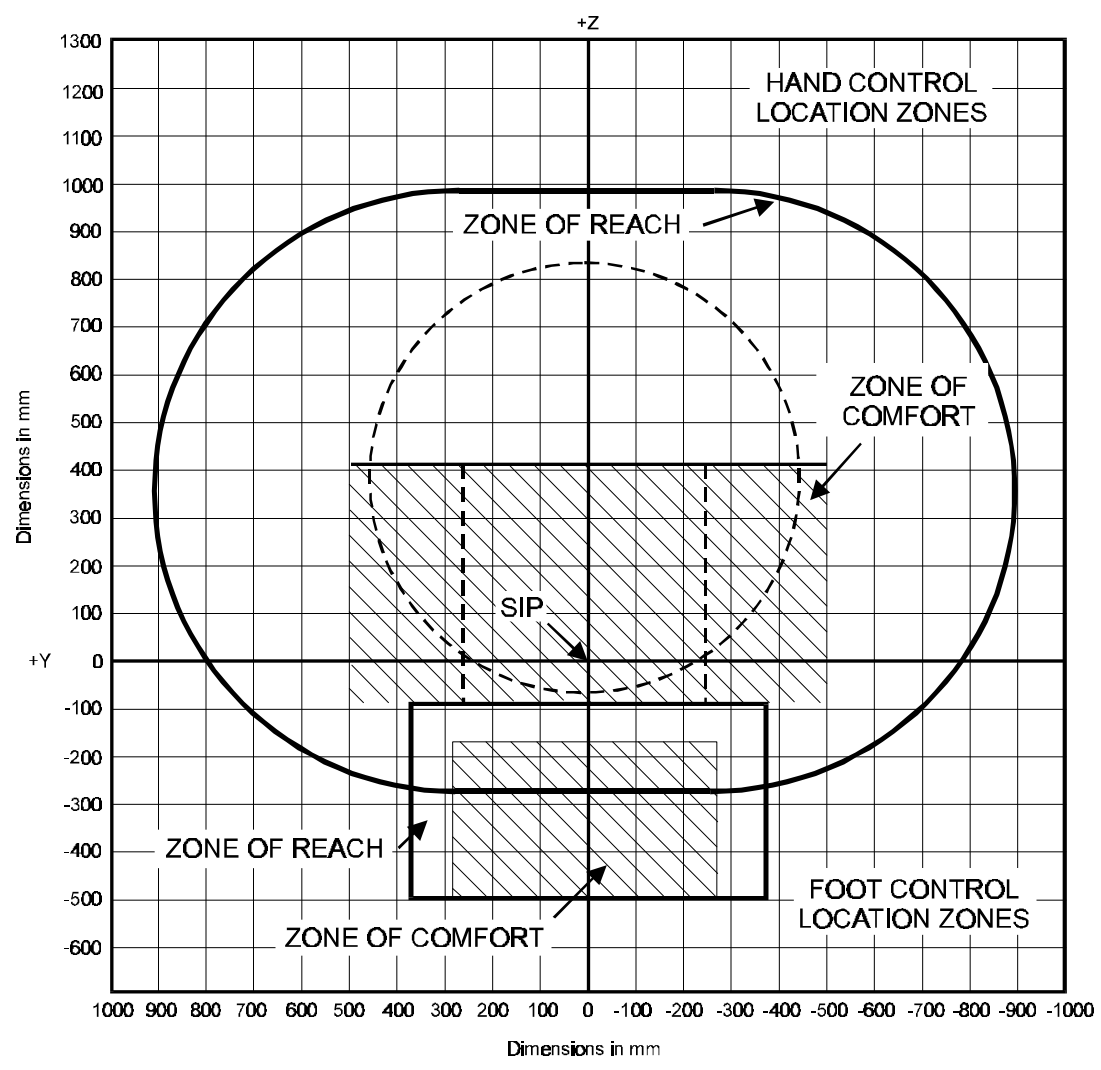

Figure 5-4. Front View - Zones of Comfort and Reach (Reprinted with permission SAE Standard J898@ 1987 Society of Automotive Engineers, Inc.)

\subsection{CONTROLS}

\subsubsection{Operational Issues}

There are many controls located in a locomotive cab. In this discussion, the controls are grouped into three basic categories, internal, external, and combined depending upon: a) where the engineer might be focusing his attention and $b$ ) the stimulus for using the control. Figure 5-5 is an organization chart of this grouping showing the typical locomotive controls. The combined category exists because it is not known a priori whether an engineer would be using the controls because of a stimulus inside the cab or outside the cab. 


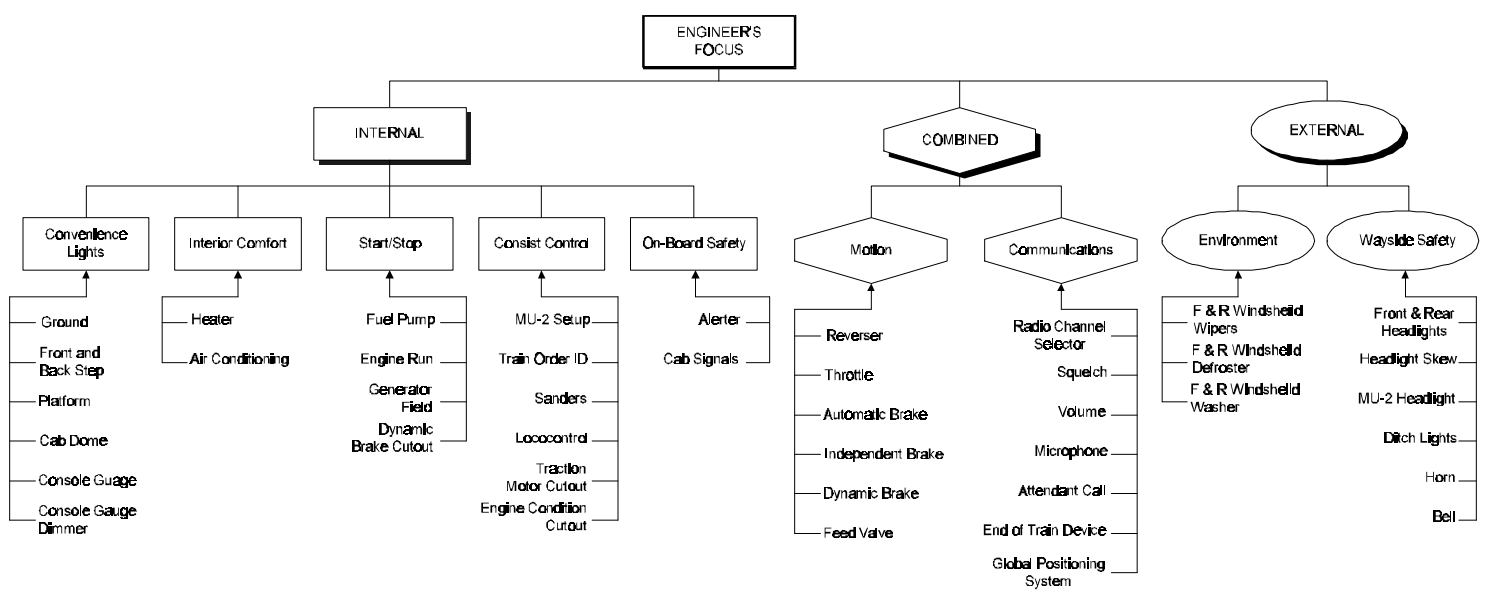

Figure 5-5. Typical Locomotive Controls

There are only 4 or 5 primary controls in a locomotive, depending upon the specific configuration being used. These are the reverser, throttle, dynamic brake, train brake, and independent brake. While these controls are all separate on the traditional cab stand, in workstation arrangements the throttle and dynamic brake are often combined into one control.

When new controls are introduced, engineers may respond by changing their behavior. For example, one engineer has reported that he tends to watch gauges more when using a continuous throttle than when using a detent throttle. This tendency might be increased if the cab speedometer is digital rather than analog.

There are two different opinions regarding the direction workstation throttle and brake controls should be moved to increase speed and braking. Both sides seem to agree that the throttle control lever should go in one direction and the brake levers in the opposite direction. The question is which way should they move.

Traditionally, to increase power, the throttle has been pulled toward the operator so that an unconscious engineer slumping forward over the workstation, pushing the throttle and brake forward, would tend to remove power and apply the brakes (Kingsley, 1980). It has also been stated that a track perturbation (due to buff forces) could cause an engineer to push the control forward if his hand were on the control at the time of the unexpected impulse. If this were to happen, with this control arrangement, he would be slowing the train not causing it to accelerate. 
On the other hand, for most non-railroad hand braking or deceleration situations, brakes are applied by pulling the controls toward the operator. Consider, for example, hand-actuated or parking brakes on an automobile, reverse throttle in boats, even the reins on a horse.

Given the principle of control motion expectancy, pushing the throttle lever forward and the brake levers back may be easier to learn and get accustomed to than having the levers move in the opposite direction. However, the transition between locomotives with different throttle orientations in a "mixed" fleet could lead to significant safety problems.

\subsubsection{Human Factors Considerations}

When specifying a control, one must first consider the intended function of the control. There are four basic kinds of functions. Bailey (1989) defines these as:

1. Activation: a binary two-position control, usually either on or off. A room light switch is an example.

2. Discrete Setting: a control requiring three or more discrete settings. The locomotive throttle is an example.

3. Quantitative settings: a control requiring continuous setting (i.e., infinitely variable through the range). The locomotive dynamic brake control is an example.

4. Continuous control: a control requiring constant adjustment. A sailboat's rudder is an example.

Foot controls. Bailey states that foot controls should be considered when moderate to large forces (greater than 20 to 30 lbs.) are required or the hands are overburdened with other tasks. Each foot should not have more than two controls assigned to it and these should only require fore and aft or ankle flexion movement.

Capability limits. Bailey further states that the force, speed, accuracy, and range of body movements required to operate a control should never exceed the capability limits of the least capable user. In fact, these performance requirements should be considerably less than the abilities of the least capable user. In 


\subsubsection{Recommendations}

addition, the control surface should be designed to prevent the finger, hand or foot from slipping.

Resistive force. Another consideration in the design of controls is the amount of resistive force a control will provide. Too little resistive force and a control might be inadvertently actuated. Too much force and the operator may quickly become exhausted or injured from operating the control.

Feel of controls. In some instances, the feel of a control may be carried over into newer designs of the same control. This may be desirable from a perspective of consistency. However, the designer must be aware that there may be time when such consistency is to be avoided. This is particularly true if the newer design operates in a different direction, has a different location, is operated by a different part of the body (hand versus whole arm, knee versus foot, arm versus leg), or has a different range of motion.

Given the dual nature of the controls in the combined branch, they should have the highest priority in their placement. The motion controls should be placed directly in front of the engineer with the brake module on the right and the reverser and throttle on the left. The radio hand controls should be placed on the left hand side to allow an engineer to operate the locomotive motion controls with his right hand while still using the radio with his left hand. This arrangement could be especially helpful when moving cars in and out of a consist. These controls should all be located within the zone of comfort for hands.

Controls for the sanders, whistle, horn, headlights, radio and microphone, should be located at least within the zone of reach and preferably within the zone of comfort, if possible.

Arrangement of controls. Controls should be arranged to minimize the requirement for engineers to change their position solely to operate a control. Also, all controls should be positioned so that, in manipulating them, operators do not appreciably move their nominal eye reference and possibly miss seeing important events occurring outside or on the principal internal display (Woodson, 1992). 
Order of controls. Controls should be arranged according to the order they are expected to be used. Tracing the sequence of control use will help identify poor arrangements. Note though, that the brake module containing the controls for the train and independent brake should be positioned for one hand operation when moving in either direction. The location will tend to favor operation by the right hand because American locomotive engineers are seated on the right side.

Controls should operate according to generally accepted control motion expectations. Refer to Figure 5-6(a) for a listing of these expectations.

\section{Figure not displayed because electronic copyright permission was not granted}

Figure 5-6(a). Recommended Control Motions (Adapted from Human Factors Design Handbook, 2nd Edition, Woodson, et al., 1992, p. 433. Used by permission from McGraw-Hill, Inc., New York, NY.)

Controls should be consistent with normal limb motions. This means that where arm motions are needed they should be forward and back, not sideways (see Figure 5-6(b)). Compare the workstation arrangement of the primary controls versus the cab stand arrangement.

Controls that have a similar function or purpose should be grouped together. Several methods can be used to reinforce the grouping such as location; shape, size, and color coding; mode of operation coding, and labeling. Care should be taken when dimensional coding is used to ensure that all engineers will be able to operate the controls and not activate another control inadvertently. This is especially important in cold northern 
climates when engineers can be expected to wear bulky clothes and winter gloves. Typically hand controls should have as a minimum $50 \mathrm{~mm}$ clearance between the control and any other control or adjacent surface (Kingsley, 1980).

\section{Figure not displayed because electronic copyright permission was not granted}

Figure 5-6(b). Recommended Control Motions for Various

Panel Orientations (Adapted from Human Factors Design Handbook, 2nd Edition, Woodson, et al., 1992, p. 433. Used by permission from McGrawHill, Inc., New York, NY.)

\subsection{ELECTROMECHANICAL DISPLAYS}

\subsubsection{Operational Issues}

Displays are essential for monitoring both the state of the train and the state of the locomotives. As stated earlier, engineers must be able to read the displays at a glance. Specifically, instrument panel layout should facilitate both rapid identification of system states (in particular, failures), and rapid identification of which system is referred to by each display (Kingsley, 1980).

The lighting conditions under which gauges are read can vary widely. The reading of gauges may also be affected by an

individual engineer's visual distance and angle to the gauges, visual acuity, color acuity, and other factors. 
Since the primary visual task of the engineer is surveillance outside the cab, then it is expedient that the most important internal displays should be positioned so that they can be viewed without excessive eye movement from the nominal exterior line of sight. The most important internal displays are the speedometer and the air brake gauges.

\subsubsection{Human Factors Considerations}

Reliability of Display Readings
A basic factor in the reliability of reading an instrument dial is the physical width (measured as an angle of view) of the subjective scale divisions (Ivergard, 1989). The subjective scale divisions are the smallest step which is necessary to interpolate. There should be zero, two, or five subjective scale divisions for every marked interval. For a scale marked in five subjective scale divisions per marked interval, and which has a total of 100 subjective scale intervals, the total length of the scale is determined according to the radius distance obtained from the following formula:

$$
\mathrm{D}=14.4 \mathrm{~L}
$$

Where:

$$
\begin{aligned}
& \text { D - Reading distance } \\
& \text { L - Length of scale }
\end{aligned}
$$

and $\mathrm{D}$ and $\mathrm{L}$ have the same units.

If the standard scale can not be obtained, a correction factor may be used which leads to the modified formula:

$$
\frac{\mathrm{D} \times(\mathrm{ix} \mathrm{n})}{100}=14.4 \mathrm{~L}
$$

Where:

i - number of subjective scale divisions into which each marking interval is to be interpolated, and $\mathrm{n}$ - the number of marking intervals.

Adjustable displays. When a display is adjustable by means of an associated control, the control should be located as close as possible to the display as practical. Since there may be directional relationships, care must be taken to position the control relative to the display. There must be no doubt as to the direction in which the control should be moved in order to cause the displayed element to move in the right (desired) direction. Controls which are placed below their respective displays are less confusing than those that are placed to the right or left of the 
displays.

Dial Size

The size of a dial or rectangular gauge normally should be determined by the number of scale graduation marks required (which is a matter of inherent precision required) (Woodson, 1992). The spacing of scale graduation marks must be great enough so that the observer can discriminate between one mark and another, and also see the relationship between the marks and the pointer, without taking an inordinate amount of time peering at the instrument. In addition, the size of the dial depends on how deeply inset the face is below the bezel. When the case design includes a deeply inset dial face, the bezel tends to limit the viewing angle, with the result that (depending upon the viewing angle) some of the numbers and/or scale marks may not be visible.

Further, too many scale marks, too close together, make dial reading difficult and error -prone. Table 5-1 provides guidance in determining the size of dial necessary to accommodate the required number of scale marks.

Table 5-1. Minimum Diameter of Inner Annulus at Various Viewing Distance

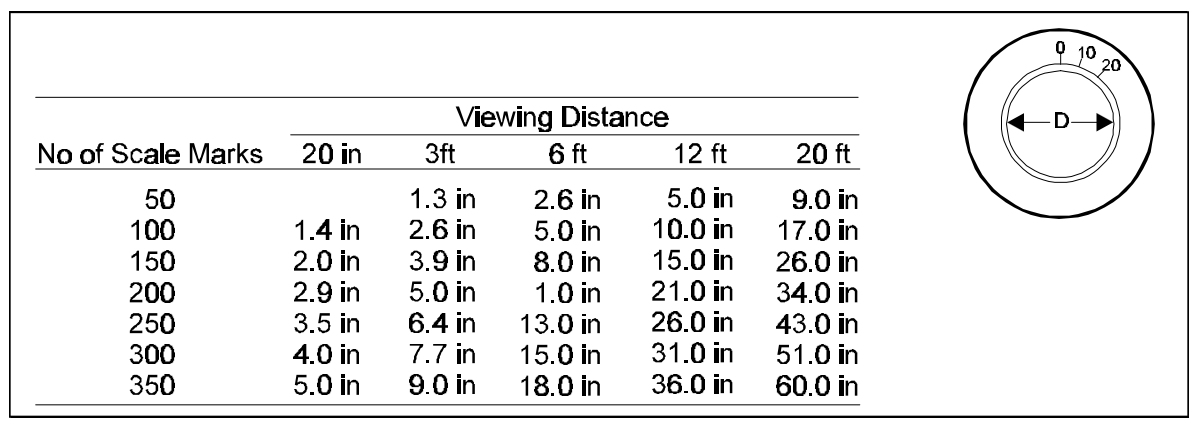




\section{Scale-Marking Guidelines}

The 28-inch viewing distance is typical of the aircraft cockpit situation (Woodson, 1992). For other viewing distances the proportions should be maintained even though values are increased or decreased. See Figure 5-7.

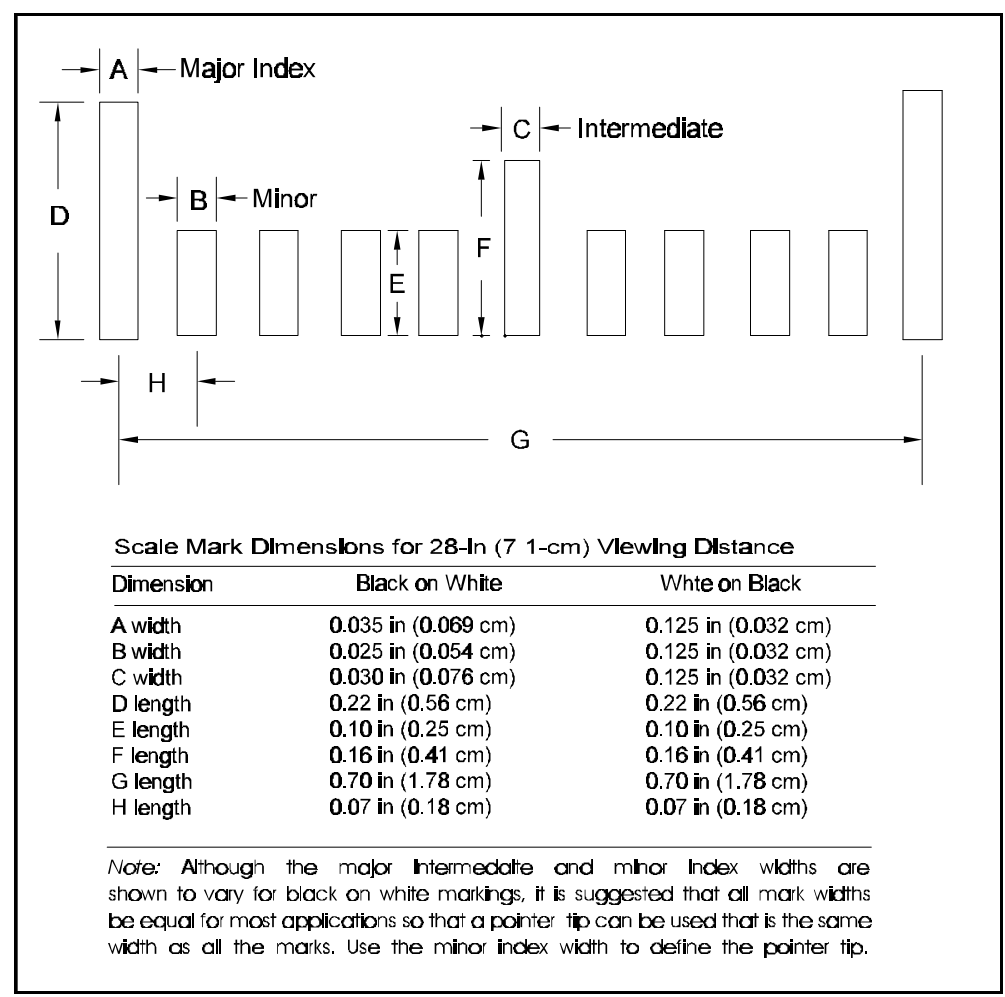

Figure 5-7. Recommended Scale Mark Dimensions

(From MIL-HDBK-759A)

Scale intervals of 1 through 10 (or multiples thereof) are easiest to interpret without error. The next easiest are intervals of 2, 4, 6, 8, and 10. Intervals such as 3, 6, and 9 (or 4, 8, and 12) can cause confusion.

Visual angle. The measurement of displayed information not only has to take into account the physical size of a character, but also the distance the character or symbol is from the eye. To account for both the size and distance of symbols and characters, the angle that the symbol or character forms with the eye is used as the unit of measurement. This is referred to as the visual angle (see Figures 5-8 and 5-9). Character size is measured by the viewing angle. However, an easy formula to use in calculating symbol or character height is that the minimum height of characters should be $1 / 200$ th of the viewing distance (the straight line distance from the eye to the character or symbol) (Cardosi and Murphy, 1995). 


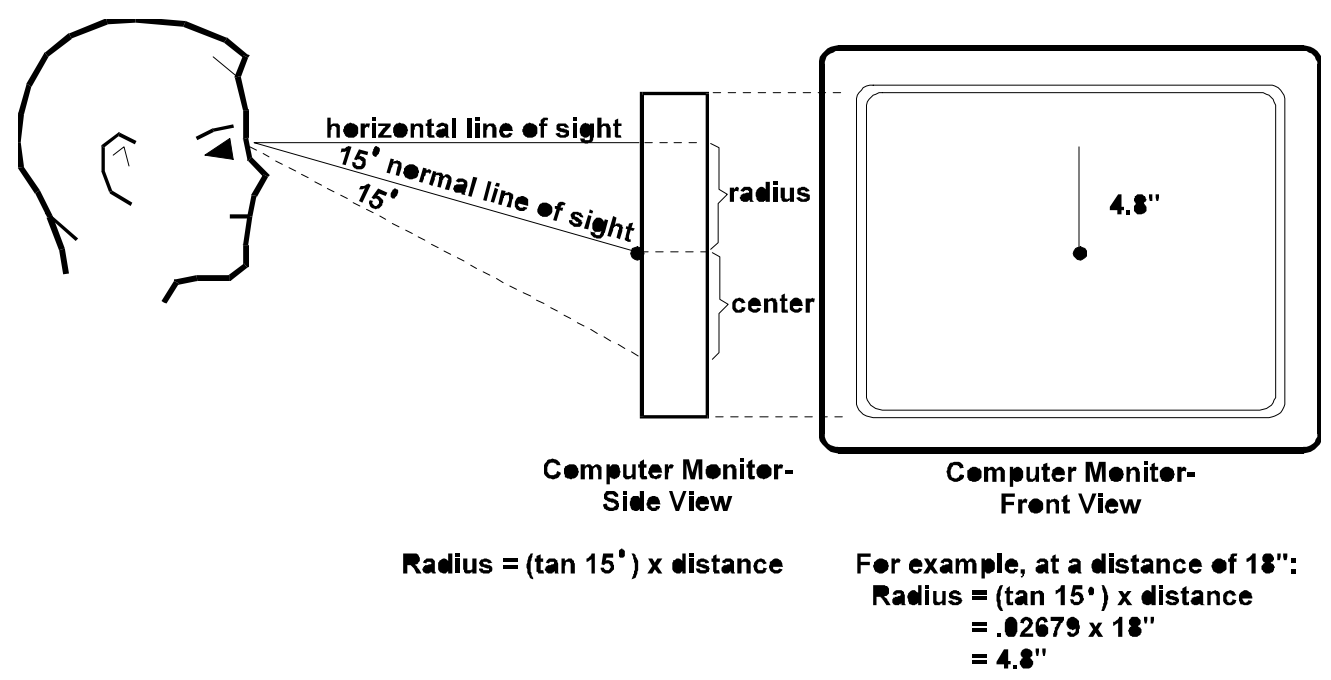

Figure 5-8. Recommended Placement of Visual Alert Signals (Adapted from Aircraft Alerting Systems Standardization Study (DOT/FAA/RD-81/38) by B.L. Berson, D.A. Po-Chedley, G.P. Boucek, D.C. Hanson, M.F. Leffler, and R.L. Wasson. 1981. Volume II, Aircraft Alerting System Design Guidelines, p. 40.)

Color of displays and visibility. For colors to be identified reliably, they must be distinct under a wide range of viewing conditions, as found in the operation of locomotives. The maximum number that fulfills this requirement is probably no greater than six. Within the central 10 degrees viewing angle, the observer is responsive to all the basic colors: red, green, yellow, blue, black, and white. As one moves out from the center, sensitivity to red and green diminishes. Thus, the zone on the locomotive ammeter, that is color coded orange to indicate dynamic braking, might instead be color coded yellow. Yet, with further eccentricity, the yellow and blue zones diminish and color responses are limited to black and white. Further, as one ages there is more difficulty in seeing the color blue (Cardosi and Murphy, 1995). However, care must be given to color conventions. Red is usually associated with an alarm indicating a dangerous situation or malfunction; yellow with a warning of an impending problem; and green with a normal operating condition. 


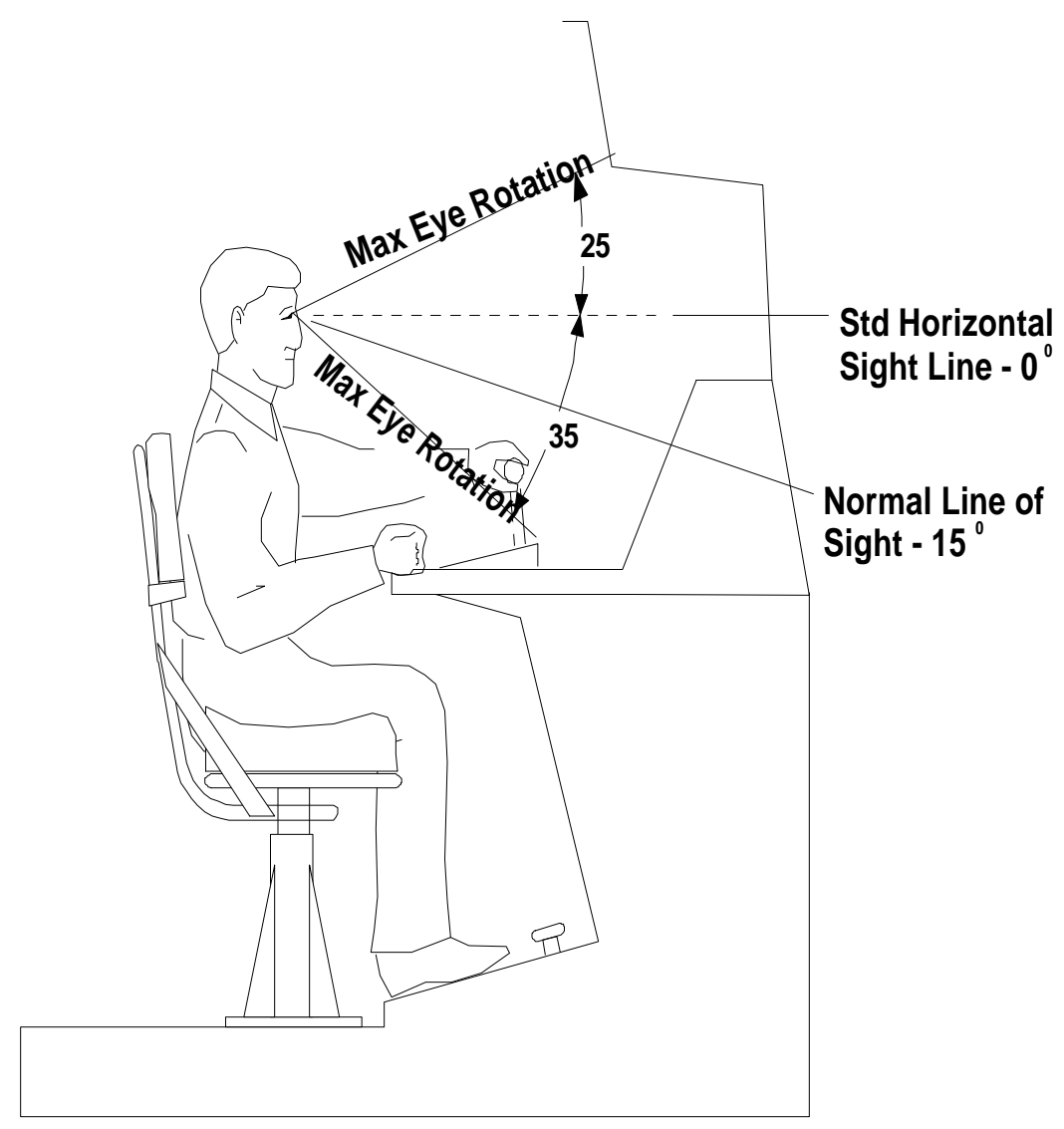

Figure 5-9. Visibility of Controls and Displays for a Seated Locomotive Engineer (Adapted from Human Engineering Guide to Equipment Design, edited by H. VanCott and R. Kincade, 1972, p. 393. Copyright by the American Institutes for Research. Adapted from MILSTD-1472D, DoD, 1989.)

Glare. Each indicator should have indirect lighting with light provided from a shielded source around its rim.

There are various methods of reducing or eliminating glare (Kingsley, 1980). These include: convex transparent dial covers, uncovered dials behind an inclined transparent panel cover, internally illuminated dials, and non -glare dial covers.

\subsubsection{Recommendations}

Order of displays. If displays are ordinarily read in sequence, arrange them in order, either horizontally, left to right, or vertically, top to bottom.

When the circumstance includes an array of displays that must be monitored on a regular basis to determine whether conditions are approximately "normal," the check -reading task (i.e., when an operator must glance at them quickly to ascertain that things are functioning properly) is made easier for the operator if the 


\subsection{ALARMS}

\subsubsection{Operational Issues}

There are two kinds of alarm systems on today's locomotives. One, the "vigilance system," monitors the human operator; the other, the engine monitoring system, monitors the equipment.

Vigilance monitoring system. Are you alert? That is the principle question behind the development of the on -board vigilance system. This system is intended to monitor the vigilance of the engineer. It provides an audio and visual cue to the engineer. If the engineer fails to respond to the cues in a timely manner an automatic brake application is made.

This system has evolved from a foot-operated deadman's switch to an elaborate system that is tied into the primary locomotive controls. Engineers are no longer forced to maintain constant pressure on a foot switch, nor are they motivated to short circuit the switch and, thereby, defeat its intended safety function as they have in the past.

Today's systems monitor engineers' use of controls. Engineers now have the freedom to change positions while still maintaining vigilance without impairing safety. The modern system is connected to a manual reset button, the throttle, all braking systems, the radio, horn, and bell (Heron, 1988). In some cases 
even window panes are part of the system.

Both visual and auditory signals are emitted if none of these controls is used within a certain time period. In some cases, this time period varies inversely with speed; as train speed increases the signaling interval decreases, and vice versa. Vigilance alarms based solely on train speed could cause a problem under conditions of low speed operation and little control activity. An engineer working under such conditions could become incapacitated and might not be detected for quite some time. However, it should be clear that "alerters" do not make fatigued engineers more alert. These systems notify the engineer that his or her alertness is falling below acceptable levels and reduce the consequences of an engineer dozing or even falling asleep while operating the locomotive.

Engine monitoring system. Another alarm system is finding its way into the modern cab. This system monitors locomotive operating conditions (see Table 5-2).

Usually this system will have both visual and auditory alarms. Visual alarms could be shown on a warning advisory panel located on the left side of the engineer through a series of indicator lights.

Both the vigilance and the locomotive monitoring system have a visual and auditory component. Each system also requires an acknowledgment from the engineer. The acknowledger control could be common for both systems since activation of any control forestalls a penalty brake application.

Table 5-2. Monitored Operating Conditions

Hand Brake On
PCS Open
Engine Overspeed
Excitation Limit Panel
Traction Motor Hot
Locomotive Overspeed
Wheel Slip
Wheel Slide
Engine Hot
Blower Failure
Sand Low

Oil Pressure Hi/Low

Oil Level Low Fuel Pressure Low Turbo Oil Pump Fail Turbo Air Pressure Low No Battery Charge Water Level Low Air Filter Clogged Oil Filter Clogg ed Fuel Filter Clogged Fuel Low

\subsubsection{Human Factors Considerations}


Response-based alarms. There are two types of alarms: response based, and stimulus based. The response based type represents the operator's alarm after interpretation of events in the outside world. In the stimulus based type, the operator responds to a specific alarm that is actuated in the outside environment.

Stimulus-based alarms. This type is needed and found in locomotives. An alarm is sounded whenever predetermined conditions are exceeded. Responses of different operators to the same alarm should be virtually the same for all operators. Otherwise, initiation of corrective action could be delayed depending on what specific stimulus might cause each operator to become alarmed.

An alarm is a messenger that provides a means of signaling state changes and a means of attracting attention.

An alarm can be visual, auditory, or both. Both types are used in locomotive cabs. Audio is used to get the engineers' attention and direct his focus to the displays that will inform about the nature of the alarm. Visual alarms then indicate which system or component has exceeded its threshold.

Auditory signals should be used when:

- The message is short and simple.

- The message will not be referred to later.

- The visual channel is overburdened.

- The message deals with events in time.

- Continuously changing information is presented.

- Vision is limited.

\subsubsection{Recommendations}

Visual signals should be placed within the engineer's visual field having the greatest visual acuity. This optimal area can be thought of as a cone extending from the engineer's normal line of sight with a radius of 15 degrees below the line extending horizontally from the center of the pupil of the eye.

It may be possible for the warning advisory panel to show alarms for all locomotives within a consist. Associated with this warning panel should be additional displays that indicate which locomotive is experiencing the particular problem. The displays for these conditions should be grouped in a logical manner that is evident simply by looking at the warning panel. This panel 
should also contain an alarm for the End of Train Unit (EOT). Lastly, a lamp test function should be built into the display panel.

When a monitored condition occurs, the appropriate warning display flashes and an audio alarm is presented to the engineer. An acknowledger control is used to silence the auditory alarm and stop the display from flashing. If the situation is corrected the panel indictor light will go out.

If the situation is not corrected within a certain time period, the audio signal resumes and the panel display begins flashing again.

Non-speech signals should be in the 200 to $5,000 \mathrm{~Hz}$ range and ideally in the 500 to $3,000 \mathrm{~Hz}$ range. Loudness of sounds used should be consistent with the ambient sound level, but not so loud that they startle or disrupt the proper response.

The purpose of the auditory display in each of the above systems is to get the engineer's attention. It should naturally direct the engineer's attention to the source of further information about the problem. For the vigilance system, the engineers attention should be directed towards the outside. Consequently, the audio alarm and visual alert should be near the windshield. For the engine monitoring system, the warning sound should come from somewhere near the warning advisory panel.

The use of sounds that could be confused with operational or malfunction noises (e.g., air brake releases, pump operations, sand discharges, etc.) should be avoided. The selection of advisory sounds should be limited to no more than four to ensure proper identification.

Two different tones should be used; the first for the vigilance system should have a sound that is indicative of urgency. If an engineer has fallen asleep this sound should wake him up. The warning advisory panel sound should be easily distinguishable from the vigilance system tone. It should be less urgent and indicated by lower frequency, lower volume, or slower pulse rate.

\subsection{DIALOG DESIGN}

This section discusses human factors issues associated with how the operator will interact with a computer-based interface for train control. Computer-based interfaces offer the designer new flexibility in creating displays and controls for exercising train control and monitoring status of the locomotive. In place of mechanical gauges, the designer may create displays that show the same information in similar formats to that found in analog 


\subsubsection{Operational Issues}

mechanical gauges or in new ways that depend upon the creativity and knowledge of the designer. Likewise, mechanical controls that were previously implemented with levers, rotary buttons and switches can now be executed using keyboards, electronic controls and other innovative devices. The issues related to electronic controls and displays mediated by computers are discussed in the following two chapters. The current section addresses how the user interface may affect the engineer.

The success with which the engineer can control the train via these input devices and gather information to stay alert to the status of the train depends in part upon how the designer conceives of the interaction between the engineer and the rest of the system. The management of this interaction is referred to as the interface style or dialog design.

The implementation of computer-based controls and displays in the locomotive cab is a relatively recent development. There is very little published research documenting the use of this technology in the railroad environment. The documentation that does exist tends to be anecdotal (Brown, 1994) and does not address the nature of the interaction and how automation might be designed into the cab. The Association of American Railroads (AAR) specifications for the operating display (AAR Locomotive System Integration Architecture Specification M591, 1993 and ATCS Specification 320, 1993) concerns itself primarily with the location of the various displays on the screen, the precision of the information to be displayed, color, labeling and the range of values that the information should take. Although not explicitly stated, the interface presented in the specification uses a menu based approach with function keys for interacting with the operating display.

Operating environment. Several issues will affect the nature of the interface that is selected for the railroad operating environment. A key consideration that will affect this choice is the environment in which the equipment must operate. The locomotive cab is a harsh environment compared to the typical office. Hardware must be able to withstand extremes of temperature, vibration, and dirt, and must be readable under exposure to bright light as well as nighttime conditions.

User characteristics and operating tasks. Two other considerations in the choice of the interface are the characteristics of the users and the tasks to be performed. For example, how computer literate is the potential user population? Many of the 
engineers who will operate this new interface were comfortable with the analog displays and controls found on older locomotives with the AAR control stand. Some of these engineers may find it difficult to adapt to a new interface if it differs radically from the old interfaces with which they were comfortable. How these issues are addressed, as well as others, will circumscribe the type of interface that will be effective in the locomotive cab.

\subsubsection{Human Factors Considerations}

The following general principles listed in Shneiderman (1992) should be considered in evaluating and developing a particular dialog design:

Support the operator's need for control. Engineers, like users of other systems, need to feel they are in control of their environment. In the case of the locomotive, it important that the engineer perceive that he or she controls the system and that the system responds to his or her actions. The system should avoid the following: actions that surprise the engineer, tedious sequences of data entries, and difficulty in obtaining necessary information. The system should include features that give feedback regarding actions taken and system responses and easy reversal of actions. Actions should be initiated by the engineer.

Aim for consistency. Consistent sequences of actions should be required in similar situations, identical terminology should be used in prompts, menus, and help screens. Use consistent commands whenever possible. Consistency in design helps the engineer to better understand how the system works and maintain situational awareness regarding what the system is doing. This enables the engineer to focus on the task at hand rather than how the interface works.

Minimize short-term memory load. Human memory is limited and the designer should design information displays that minimize the engineer's memory load. The user can generally remember 7 plus or minus 2 chunks of information. Therefore, it is critical that display clutter be minimized.

Facilitate recovery from errors. Where possible, the system should be designed to prevent errors from occurring. Total error prevention is never possible. The system should minimize the effect of errors by making them difficult to commit and by making it easy to recover from them when they do occur. The system should detect the error and offer simple comprehensible methods for handling the error. 


\section{Dialog Design}

There are four types of interaction styles that can be used alone or in some combination with each other. These include menu selection, command language, form fill-in, and direct manipulation.

Menu selection systems give the user a list of items from which they select the one most appropriate to their task.

Command language systems require the user to learn a set of commands with a specific syntax. They use these commands to initiate tasks.

Form fill-in systems are useful in situations where data entry is important. Users enter data in a series of fields by moving a cursor among the fields and typing in the appropriate information. In this system, the user must understand the labels associated with each field and be aware of the permissible values.

Direct manipulation systems create a visual representation of the tasks that can be executed. These objects can be directly manipulated by the user using pointing devices like a mouse or a touchscreen.

Table 5-3 shows the advantages and disadvantages for each of the four interaction styles.

In deciding what type of interaction style to choose for the locomotive cab, a variety of considerations to be taken into account include: minimizing head-down time, using hardware that can withstand the environment conditions found in the locomotive cab, and workload. Currently, it is important for the engineer to direct his attention out the window to monitor track signals, look out for trespassers and motorists, and determine location on the track, relative to the final destination. The interaction style selected must minimize the amount of time requested for monitoring information displays and initiating actions in response to changing conditions. Form fill-in and command languages require more typing and thus more headdown time. Both of these interaction styles also increase memory load on the engineer by requiring him to know what command to enter or how information should be typed into a field. Menu and direct manipulation systems enable the engineer to select actions and respond to changes in system status relatively quickly. Both these interaction styles also demand less of the engineer's shortterm memory. 
Table 5-3. Advantages and Disadvantages from Four Interactions Styles

(Adapted from Shneiderman, 1992.)

\section{Interaction Style}

\begin{tabular}{|c|c|}
\hline Advantages & Disadvantages \\
\hline $\begin{array}{l}\text { Menu selection } \\
\text { shortens learning } \\
\text { reduces keystrokes } \\
\text { structures decision making } \\
\text { permits use of dialog-management tools } \\
\text { allows easy support of error handling }\end{array}$ & $\begin{array}{l}\text { imposes danger of many menus } \\
\text { may slow frequent users } \\
\text { consumes screen space } \\
\text { requires rapid display rate }\end{array}$ \\
\hline $\begin{array}{l}\text { Command language } \\
\text { flexible } \\
\text { appeals to "power" users } \\
\text { supports user initiative } \\
\text { convenient for creating user-defined macros }\end{array}$ & $\begin{array}{l}\text { poor error handling } \\
\text { requires substantial training and } \\
\text { memorization }\end{array}$ \\
\hline $\begin{array}{l}\text { Direct manipulation } \\
\text { presents task concepts visually } \\
\text { easy to learn } \\
\text { easy to retain } \\
\text { allows errors to be avoided } \\
\text { encourages exploration } \\
\text { permits high subjective satisfaction }\end{array}$ & $\begin{array}{l}\text { may be hard to program } \\
\text { may require graphics display and } \\
\text { pointing devices }\end{array}$ \\
\hline $\begin{array}{l}\text { Form fill-in } \\
\text { simplifies data entry } \\
\text { requires modest training } \\
\text { makes assistance convenient } \\
\text { permits use of form-management tools }\end{array}$ & consumes screen space \\
\hline
\end{tabular}

It should be emphasized that the menus and direct manipulation are not mutually exclusive interaction styles. Direct manipulation can include menus. For example, a menu of response alternatives may be presented textually or graphically or in some combination. Direct manipulation differs from menu systems in how the item is selected. In menu systems, function keys or keys on the keyboard activate the item to be selected. In direct manipulation a pointing device (i.e., finger or mouse) activates the item to be selected.

The interaction style currently recommended for use by the AAR (Locomotive System Integration Architecture Specification M591, 1993 and ATCS Specification 320, 1993) is a function key menu-based interface. Specifically, it adopts a soft function key approach. 
According to Mayhew (1992), this interface has the following advantages: it tends to be self-explanatory, requires little human memory, is easy to use, flexible, and has low typing requirements. Soft function keys accommodate richer functionality than hardwired function keys, but increase complexity and use more screen space. One of the advantages of soft function keys, the ability to quickly and easily add functions, can become a liability as the increasing functionality increases the complexity of the system. As the systems grows in complexity, it becomes more difficult to learn and use.

The interaction style can be thought of as a metaphor for how the user interface works. For example, the Apple Macintosh ${ }^{\mathrm{TM}}$ computer uses a desktop metaphor to represent how the system works. To open a file located in a specific directory, the user selects a folder containing documents. Selecting one of these documents opens the file. The construction of the user interface represents a road map for how the system works. The effectiveness of the user interface depends upon how well the representation of the system, say a menu system, matches the engineer's way of thinking. According to Norman (1991), the layout of the system should engage the way the user conceptualizes the operation of the system. Menu systems frequently hide the organization and structure of the system. Matching the representation of the system to the way the operator processes information makes it easier to learn. Learning is facilitated when new information can be assimilated into an existing framework.

For menu systems as well as other types of user interfaces, usability depends upon how it is organized. As a system grows in complexity, the importance of good organization increases. Good organization is a function of the amount of information displayed and the formatting of that information.

Since the AAR specification uses a menu based user interface, particular attention is paid to human factors considerations in the design and evaluation of this type of interface. However, the same information processes that affect performance using menus also influence performance in other types of user interfaces. These processes include searching for information, encoding and decoding the meaning of response alternatives, assessing and choosing from the response alternatives, and making a response. 


\section{Information Search}

To speed the search for information, the designer must make the organization of the menu or user interface as transparent or obvious as possible, to the user. For menu systems, the designer must make trade-offs between breadth and depth. For the system with many choices, the designer must decide whether to display all the items on one screen or organize the information on several screens. The effectiveness of a broad menu depends upon the number of items. The more items there are, the longer it takes to search the menu. A menu with a deep structure may have less information on each screen, but it may be buried deeper within that system. The deeper an item is within the menu, the more the operator must go through the process of searching, deciding on a choice and making the response selection to get to an item. The time spent on each screen may be less than with a broad menu, but more screens must be encountered (Norman, 1991). Deep menus place greater memory requirements on the user in order to remember where an item is located.

Current research suggests the following breadth versus depth tradeoffs (Norman, 1992). Increase the breadth to the maximum practical level for linearly organized lists such as alphabetized lists, months of the year, letters of the alphabet. When there is no inherent linear ordering of alternatives, the optimal number of alternatives will fall between three and twelve. Response time can also be reduced with broader menus, if multiple levels of a hierarchical menu can be displayed in one screen. Where possible, menu trees should be limited to three levels to reduce the likelihood of getting lost or disoriented (Shneiderman, 1993). Within each screen or menu, the designer is faced with the issue of how to organize the response selection to facilitate quick access. A number of principles may guide the designer. These principles include organizing by logical grouping and frequency of use, chronological ordering, and importance of items. While it may seem obvious that organizing menu items by frequency of use should offer the fastest access time, the designer's task may be complicated by the fact that several tasks are performed in which the frequency with which selected menu items differs. Furthermore, as engineers progress from novice to experts in their use of the menu system, they will be less tolerant of slower access times to low frequency items. The solution is to provide rapid access methods by which the engineer can go directly to a given menu (Shneiderman, 1993; Norman, 1991).

Regardless of the interaction style, the designer should 


\subsubsection{Recommendations}

standardize the location of information elements to aid the development of expectancies about where that information is located and decrease response time. This means that displays like the speedometer, air brake pressure, or menu system should be located in a fixed position on the display screen or workstation.

\section{Response Selection}

In constructing the dialog design, the designer should seek compatibility between controls and displays. The idea that the selection alternatives are compatible or congruent with the method for implementing them is an important human factors principle. For example, the response alternatives on a menu may be arrayed horizontally from left to right. The control-display relationship would be considered compatible if the response keys were also arrayed from left to right.

\section{Evaluation Guidelines}

Since AAR specification proposes the use of a soft function key menu interface, the following guidelines specifically addressing this type of interface are offered (Brown, 1989; Mayhew, 1992; Smith and Mosier, 1986). Optimizing a soft key function menu interface, designers need to consider a number of issues: spatial layout, labeling, and consistency.

Provide enough function keys to support functionality, but not so many that scanning and finding are difficult. In evaluating the number of function keys, the amount of screen space and keyboard space to allocated function keys will need to be considered. There is no rule of thumb for the number of keys that are appropriate. However, as the number of keys increases beyond a certain level, the keyboard or workstation and display will become increasingly crowded, making it more time consuming to search for the relevant response selection and implement that selection.

Spatial layout. Arrange keys in groups of three or four based on semantic relationships. Figure 5-10 shows two function key interfaces. The top design lacks a meaningful grouping and contributes to difficulty in locating a specific key. The bottom design logically arranges keys into several groups by function and facilitates learning and ease of remembering where different keys are located. 


\section{Poor layout}

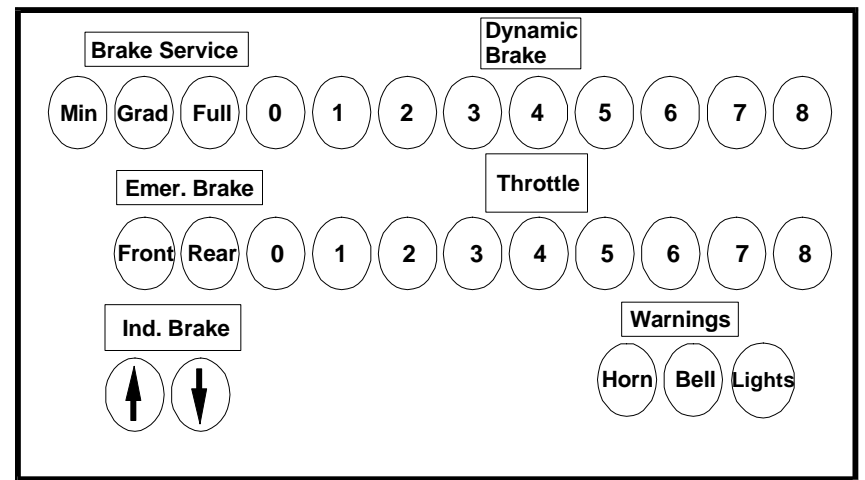

\section{Better layout}

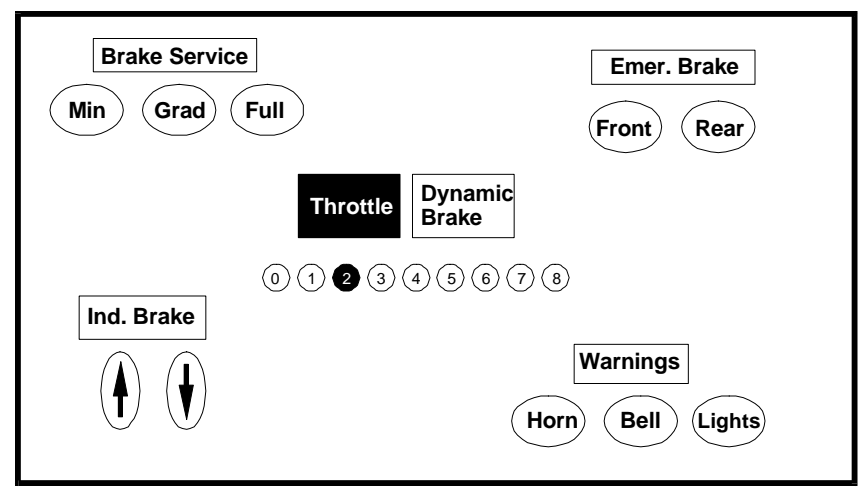

Figure 5-10. Poor and Improved Function Key Design

Coding schemes. Use space, size and color to distinguish groupings. Using space, size and color to differentiate logical groupings will facilitate the search for specific keys and aid memory for the meaning of the different keys. For example, the designer could place function keys that have a destructive effect away from the main keys to avoid accident activation. Placing keys with potentially destructive effects in hard to reach places makes it less likely the engineer will press it by mistake. Keys could also be color coded to be consistent with user expectations (i.e., green for go, red for danger or warning).

Labels. Label keys clearly and distinctively. Since there is frequently a shortage of space on the key or screen, abbreviations may be necessary. A consistent rule should be adopted for assignment of function key names such as truncation or vowel deletion. However, when a rule produces a name that is less likely to be recognized than an alternative name (e.g., Search is probably more recognizable than sea for search, while del is probably more recognizable than dlt for delete).

Frequency of use. Place the most frequently used keys in the 
most accessible positions. Within a set of function keys, some keys may be more accessible than others. For example, in a single row of keys, the keys on the end are more accessible than the keys located in the interior. Place the most frequently used keys in these positions to speed response time.

Qualifier keys. Minimize the use of qualifier keys. It is frequently difficult to develop a meaningful method for assigning key sequences using qualifier keys (i.e., Shift, Alt, Command, Control). The lack of useful memory aid for assigning key sequences increases the memory load on the engineer. Multiple key strokes are also more prone to error. However, when qualifier keys cannot be avoided, use them consistently. Consistency in logic helps to learn the functions associated with that key.

Control-display compatibility. Preserve spatial relationships between soft function key labels on the screen and generic function keys on the keyboard. Consistent relationships between the display and the control are essential to foster learning of the system, for ease of use, faster performance, and to minimize errors. Use the same coding schemes (space, size, grouping) for both the displays and controls.

Consistency in key assignment. Be consistent in function key assignments across screens and subsystems. Smith and Mosier (1986) recommend that when a function key performs different functions in different operational modes, the designer should assign equivalent or similar functions to the same key. For example, a particular key might confirm data changes when operating under ATCS and confirm message transmission when in a non-ATCS environment.

Feedback. The system should give the user feedback regarding the status of function key operation. When function keys are pressed, the system should give the operator feedback to indicate whether some action took place and how the system is responding. This may include visual, auditory and/or tactile feedback. Where key labels remain the same, some system

change takes place that makes a particular menu selection inoperative, the inactive key or menu selection should be grayed out or deleted to indicate this change. If there are special keys that have different modes (e.g., Caps Lock), a status indicator should indicate what mode the keys are in. For example, a light on the function key could indicate for which mode the function 
key is set by whether the light is on or off.

Navigation. Enable easy access to the home menu. In hierarchical menu systems, with multiple levels, the user may need to quickly return to the home menu to initiate a new sequence of actions. If the functions assigned to a set of keys changes as a result of user selection, give the user an easy way to return to the home menu.

\section{Response Time Performance}

The time taken by the system to respond to the engineer's commands may affect performance in a number of ways. Shortterm memory is limited by how long information remains in memory, generally 15-30 seconds. Short-term memory is also highly susceptible to interruptions, resulting in loss of information. When response time is too slow, engineers who are engaged in a complex information processing activities may forget pertinent information, contributing to operator error or delays in completing the task. Response times that are too short can also contribute to operator error when users try to work too quickly (Shneiderman, 1992). Without enough time, users may fail to properly plan their course of action and make performance errors.

Shneiderman offers the following suggestions for controlling system response time:

Response time should be appropriate to the task. Users prefer short response times. Response time longer than 15 seconds may become disruptive. Table 5-4 shows suggested response times:

Table 5-4. Suggested Response Times by Task

\begin{tabular}{ll}
\hline Type of Task & Time (Sec.) \\
\hline Typing, cursor motion & $0.05-0.15$ \\
Simple frequent tasks & 1 \\
Common tasks & $2-4$ \\
Complex tasks & $8-12$
\end{tabular}

The engineer's expectations about response time also affect performance. Engineers, like other users, have expectations based upon past experience about how long certain events should take. These attitudes color the perception of whether a response time is considered too fast or too slow and the significance of this perception (Shneiderman, 1992). Actions completed faster than expected may be perceived as having not occurred. If the action 
takes much longer than expected, users may become frustrated with the slowness of the system or worry that something is wrong with the system.

User expectations of acceptable response time varies with the individual's tolerance for delays. These variations are influenced by the individual, the task and environmental factors (Shneiderman, 1992). While people may adapt to changes in response times, their performance may suffer.

Extreme variation in response time should be avoided where possible and acknowledged by the system where it is not possible. For unusually fast or slow response times, the system should give the engineer a message explaining what is happening. The operator who experiences response times $50 \%$ faster or slower than expected may become anxious about the status of the system.

\section{Error Management}

In managing errors, the first goal is to prevent their occurrence. The system should be designed to prevent the engineer from committing errors wherever possible. For example, in locomotives with AC traction motors, putting the throttle into reverse when it is moving in the forward direction will damage the motors. The system could prevent this from happening by not allowing the engineer to execute this command when this condition occurs or delaying its execution until the conditions are appropriate. Where the engineer must engage in operations that are difficult or impossible to undo (i.e., destroying data) the system should notify the engineer and require confirmation before executing the command (Smith and Mosier, 1986).

Where errors do occur, the system should enable the user to recover from them quickly and easily (Norman, 1991). A responsive system will indicate the nature of the problem and suggest how to correct it. An undo function should be provided for reversible actions (Smith and Mosier, 1986). When a command must be reentered to correct an error, the system

should prompt the user to reenter that portion of the command that needs correction. Do not require the entire command to be reentered.

The system should also allow engineers to back out of a sequence of commands or to change previously entered selections. 


\section{Guidance \& Feedback}

Alarm design. When errors occur, the system should tell the user what happened and how to correct it. The system should also notify the engineer when changes in the system require some corrective action. Alarms or alerting signals serve this purpose by attracting the engineer's attention and indicating the state of the system. A number of issues need to be considered in the design of alarms.

The locomotive is a complex environment with the engineer's attention divided between looking out the window and monitoring displays and controls inside the locomotive cab. Currently, the engineer's primary attention is directed out the window with secondary concern with the visual displays in the cab. The system should not overload the user with too many alarms that may distract the engineer from looking out the window.

Auditory and visual alarms. The use of auditory and visual alarms both should be considered both alone and in combination. Visual alarms are advantageous when the engineer can selectively attend to the alarm. They can indicate more precisely the nature of the problem and possibly what actions are necessary. Visual alarms using text can be understood more quickly than speech signals and relies less on short term memory. They are also advantageous when the ambient noise level is high.

Auditory alarms. Auditory alarms are preferable under the following conditions (Sanders and McCormick, 1993):

- The message is short and simple.

- The message will not be referred to later.

- The visual channel is overburdened.

- The message deals with events in time.

- Continuously changing information is presented.

- Vision is limited.

Degree of warning. An alarm conveys varying degrees of warning or danger (Stanton, 1994). For example a low fuel warning is less urgent than a brake failure warning. Alarm design should convey the urgency of the warning so that the engineer can allocate his attention appropriately, particularly if multiple warnings are present. The perceived urgency of an auditory warning can be varied by changing speed, number of repeating units, and speed of the signal (Edworthy, 1994 in Stanton, 1994). The perceived urgency of the visual warnings can be varied by 
changing the color and flash rate of the signal (Sanders and McCormick, 1994)

Give the engineer control over the alarms by providing a means to acknowledge and turn off non-critical alarms. Where alarms are presented by both visual and auditory methods, allow the engineer to turn off the auditory alarm, without erasing the visual message that accompanies the auditory signal (Smith and Mosier, 1986).

Another method to give the engineer control over the alarm is to provide a mechanism for the modifying the conditions that activate the alarm (Smith and Mosier, 1986). False alarms that occur too frequently can result in the engineer ignoring the alarm altogether. Except where functional, procedural or legal requirements preclude user control, allowing the engineer to define when an alarm activates may reduce the likelihood of an alarm being ignored. For systems that allow the engineer to define alarm boundary conditions, the system should show the status of those settings when requested.

\section{Feedback and User Guidance}

Feedback gives the engineer information about the condition of the system and the steps necessary to change the system (Williges, Williges, and Elkerton, in Salvendy, 1987). As a general rule, feedback should provide some indication of the system status to users at all times and occur close in time to a related event. Three system states should be considered (Norman, 1991): when the system is waiting for the user to initiate an action; when the user has selected a response, but not implemented it; and when the user implements the selection. Feedback should clearly distinguish between the system that is waiting for the user to initiate an action and a delay in the response to an action initiated by the user.

When the results of user action are contingent upon different operation modes, clearly indicate the currently selected mode.

Every input or response selection by the user should consistently produce perceptible response output from the system.

When errors occur, feedback should clearly indicate the corrective action to take. The following guidelines address the formatting and content of error messages (Shneiderman, 1992; Smith and Mosier, 1986; Williges, Williges, and Elkerton, 1987). 


\section{Error Messages}

Error messages should be specific (task oriented), concise as possible, and written from the user's perspective. Use language that the engineer will understand. The visual format and placement as well as the grammatical form, terms and abbreviations should be consistent throughout the system.

Use the active voice and a positive tone to tell the user what needs to be done.

Consider multiple levels of messages where users may desire more detailed levels of information or where more than one error was made.

Display the error message after the user completes a response to minimize disruption of the user's task performance and thought processes.

\subsection{AUTOMATION}

Display error messages within 2-4 seconds following the response for which the error is detected.

This section discusses the use of automation in the locomotive cab and how it may affect the engineer. Automation is defined as the use of machines, electronic devices, and computers to perform tasks previously completed by engineers. The goals of automation are reviewed and the human factors considerations that need to be considered as automation is incorporated in the locomotive cab are explored.

\subsubsection{Operational Issues}

New command, control and communications technology is being considered for improving railroad operations. These systems include: Advanced Train Control System (ATCS), Advanced Railroad Electronic System (ARES), and Positive Train Control (PTC). The goal of these systems is to improve train Operations through the following processes (FRA report to Congress, 1994):

- Ensuring positive train control.

- Maintaining flexible blocks.

- Enhancing train management.

- Improving accuracy in train communications.

- Maintaining constant communication.

- Providing information to the locomotive engineer. 
These new systems may change how the engineer interacts with the controls and displays as well as the tasks the engineer performs. For example, positive train control operations could override the engineer's controls by braking the train to enforce speed restriction or avoid collisions and obstructions. Digital radio will enable the dispatcher to send movement authorities directly to on-board computer in the locomotive cab and display this information directly. Eliminating the need to hear and record this information transmitted by voice radio will eliminate a source of human errors due to hearing or transcription.

However, automation carries risks as well as benefits. Automation in the locomotive cab can potentially impact safety by decreasing situational awareness or increasing operator workload. The extent to which automation improves safety and productivity depends upon how it is implemented. Situation awareness is the perception and comprehension of elements in the environment and the projection of their status in the near future (Endslay, 1992).

Automation in train control is currently under development. None of these promising systems is in actual freight operation in the U.S. The only Federal government sponsored study evaluating ATCS found this new system to improve train control when compared to conventional train control systems (Keuhn, 1992). The use of predictor displays was established as an effective tool to aid in train control. However, this study evaluated only one aspect of automation. Other issues that need be considered as automation is developed for train control include: the type of errors made, impact on situation awareness and workload, and the use of automation to manage information.

As part of the development effort, the AAR has developed specifications for building an ATCS. The specifications address equipment compatibility to insure that data communications flow smoothly from one source to another (Report to Congress, 1994). Human factors concerns are addressed primarily with regard to where information should be displayed, e.g., message content and display parameters such as color and range of value the display should take. The specifications do not explicitly address what level of automation will be included, who is in charge, and other concerns related to automation. These issues are discussed in the next section.

For automation to be successful, it must be accepted by its users. 
Whether engineers find automation acceptable will depend upon a number of considerations that include: the quality of information, training and the relationship of the engineer to the automation. If the automation provides additional information for train control in a way that increases safety and efficiency, it is likely to perceived positively. However, if the automation is perceived as replacing the engineer and he or she becomes subordinated to the system, automation is more likely to be viewed negatively. The key is who is in charge of the system.

Training and skill level necessary to operate an automated system will also affect user acceptance. Anecdotal reports suggest that some engineers may receive insufficient amounts of training before operating the newer model locomotives with computerized displays and controls. While the primary group receiving training are the engineers working in main line freight operations, training should also be considered for other users, such as those involved in switching, yard, and maintenance operations.

\subsubsection{Human Factors Considerations}

\section{Task Performance}

The introduction of computers and improved communication technology gives the railroad community the opportunity to rethink the duties engineer undertakes. Previous studies have identified the tasks performed by the engineer (Hale and Jacobs, 1975; and Railroad Engineman study, 1972). This information can be helpful in reallocating functions within the cab between man and machine according to the strengths and weakness of each component. Functions that humans tend to perform poorly, such as vigilance activities, can be automated or performed by the system. Handling unpredictable events for which the system is not equipped may be given to the engineer.

However, this approach to function allocation by itself, is not sufficient. Bainbridge (1987) points out that the designer must also consider how to maintain human performance by supporting the engineer's skills and motivation.

In a fully automated train control system, the role of the operator can change from an active controller to a passive monitor. This should be avoided by assigning the engineer an important role. For example, the German Intercity Express (ICE) train has a fully automated speed control system. In this system, the engineer's 
primary attention is focused on in-cab displays to monitor and diagnose various control and braking systems failures (Sheridan et al., 1994). When failures are predicted or occur, the engineer must select, develop and execute strategies to deal with the problem. In the ICE system, the engineer is provided with technical training and elaborate instrumentation and computerbased "expert systems" support. This highly automated system has many advantages: it permits the use of a virtual (electronic) block systems which enhances system throughput; and supports the use of regenerative braking which enhances energy efficiency. By contrast, the French TGV trains use a control system which emphasizes human control with computer monitoring and assistance (Sheridan et al., 1994). The strong reliance on in-cab displays will lessen situational awareness with regard to the outside environment. Here the engineer is in charge of train control. In controlling the ICE system, the engineer may have difficulty if the automation fails. The lack of practice results in a loss of skill level, making it more difficult to regain control.

Furthermore, a failure of the automation is likely to result in a high workload situation due to the conditions causing the failure and the additional activities required to exercise manual train control (Bainbridge, 1987).

Automation affects the engineer's task performance in a variety of ways. It impacts the engineer's workload, situational awareness, and the type of errors committed and job performance. Whether the impact is positive or negative will depend upon how the automation is implemented.

Automation can reduce workload by relieving the engineer of tasks previously performed by the engineer. This includes monitoring, decision-making, and control tasks. The danger lies in reducing the workload level to a point where the engineer becomes disengaged from the operation of the task.

Automation and computer systems are well suited to performing routine, repetitive tasks. Relieving the engineer of these task may increase the precision and reduce the errors associated with these type of tasks, but they may also contribute to a lack of situational awareness. These routine tasks help the engineer engaged and aware what the system is doing. Lacking the need to act under low workload conditions, the engineer may be more susceptible to boredom and fatigue. Billingsley (1991) recommends that automation would better help the operator by providing greater assistance under high workload conditions and less assistance 
under low workload conditions.

Automation can increase workload by providing more information than the engineer can handle. The proliferation of displays in the cockpit (Cardosi and Murphy, 1995) make it more difficult to attend to the relevant information. As more information is displayed in the locomotive cab, the likelihood of information overload will increase. For this reason, Billingsley (1991) recommends that automation should assist in directing the operator's attention to the most relevant information.

\subsubsection{Recommendations}

The development of automation for train control is still in an early state of development. How much automation to incorporate is still an open question. Sheridan et al., (1994) suggest an incremental approach in which the level of automation for speed control progress in stages. Sheridan et al., (1994) propose four levels of automation for high speed rail operations.

- Manual control with traditional displays only. This approach incorporates no automation. The engineer is in charge of the system and uses conventional displays.

- Manual control with an integrated display. This is the lowest level of automation. The engineer manually controls the train with the aid of an integrated display.

- Manual control with an integrated display and automatic control option. This is a middle level of automation. The engineer controls the train with the aid of an integrated display, but also has the option of automatic control (i.e., cruise control).

- Fully automatic control with emergency override. The automatic control system is responsible for train control, but the engineer can override the automation.

An integrated display refers to an information display that synthesizes information elements from several displays onto a single display that form a representation that is different from information shown by the individual elements alone. It is not simply the combination of elements shown on many displays onto one display. The display provides a higher level picture of the system status.

Sheridan et al., (1994) propose that automation start with full manual control and move toward gradually increasing levels of automation. This gradualist approach is consistent with the 
evolutionary nature of locomotive development.

\section{Principles of human-centered automation}

The human factors principles listed below (Billingsley, 1991) are driven by a user-centered approach to automation in which the computer is subordinate to the engineer. A user-centered approach supports the engineer's need for information. This approach contrasts with a technology-driven approach in which the engineer is subordinate to the machine.

Evaluating the effectiveness of automated systems in the locomotive cab will require looking at a variety of issues. Currently there are few specific guidelines. A thorough evaluation will require measuring usability, suitability and acceptance by locomotive engineers. The following principles are offered based upon research in the aviation industry (Billings, 1991).

- The engineer must be in command. Since the engineer is responsible for the safe operation of the locomotive, he or she must have the ability to control those operations.

- The engineer must be an active and informed participant. To command effectively, the engineer must have an active role in controlling the train. This means engaging in relevant activities so that situational awareness is retained. The engineer must be aware of what the automation is doing to remain aware.

- Automated systems must be predictable. The engineer must be able to predict how the locomotive will be affected by the automation to know how to use it, and it must behave in a consistent manner to know when a failure occurs.

- Each element of the system must have knowledge of the others' intent. The human operator must be able to monitor the automated system. Automated systems must be able to monitor the human operator. Knowledge of each other's activities will help to prevent and recover from errors.

The following guidelines for automation of control tasks and information management support the principles listed above (Billingsley, 1991).

\section{Control tasks guidelines}


System operation should be easily interpretable by the operator to facilitate detection of improper operation and diagnosis of malfunctions.

The automation should perform the task in a way familiar to the user. When the automation fails it should be announced so that the operator takes over active control of the system.

The automation should prevent peak levels of task demand from becoming excessive. Design control automation to be of most help during time of highest workload and somewhat less help during time of lowest workload.

Meaningful duties should be provided to maintain operator involvement and resistance to distraction, if automation reduces task demands to low levels. This could be done by requiring management by consent instead of management by exception.

Control automation should provide the engineer with an appropriate range of control and management options.

A way should be provided to check the setup and information input to automatic systems.

\section{Information management guidelines}

All displays should contribute to and maintain situational awareness.

Less information can be generally better than more information, if it is the right information for a particular circumstance. While it is important to keep the engineer situationally aware, it is important to avoid giving too much information. Too much information makes it more difficult to attend to the most important information. The information that is available should be coded to emphasize its relative importance. Good formatting will help the engineer to more quickly find the relevant information.

Displays that have multiple modes should clearly indicate what mode is active.

\subsection{ELECTRONIC (COMPUTER GENERATED) DISPLAYS}

This section discusses the use of electronic, computer generated displays in the locomotive cab. Currently, electronic displays are 


\subsubsection{Operational Issues}

found in cabs that adopted the North American Comfort Cab design as well as more recent designs. In this design, computer generated displays share space on the workstation with analog electro-mechanical displays. In the future, information presented on electromechanical displays may be entirely generated on electronic computer-generated displays.

The locomotive cab is a harsh environment for electronic displays. The displays must withstand high and low ambient temperature, vibration and electromagnetic interference. The engineer needs to view the display under a varying light levels that range from very bright to very dim. Glare from direct and indirect sunlight may interfere with the engineer's ability to see the display. Because of the visibility requirements, large windows that allow sunlight into the cab contribute to the problem of glare. In addition to glare and ambient lighting conditions, the designer must consider the following factors: color, contrast, viewing angle, image quality and size.

The two basic types of display screens currently manufactured, cathode ray tubes (CRTs) and flat panel displays cannot yet meet all the environmental and visual requirements for locomotive cabs (Karbowski, Ben-Yaacov, and Blass, 1991). However, the technology is improving rapidly. For designers, the choice of an appropriate hardware platform depends upon the relative importance of various environmental and visual requirements. Karbowski, Ben-Yaacov, and Blass, (1991) favor the flat panel display over the CRT because they are more rugged and compact, and can better withstand the range of temperature, humidity, shock and vibration found in the locomotive cab.

\subsubsection{Human Factors Considerations}

This section explains the human factors concerns for displaying the computer generated information that the engineer will use for train control. The design of computer generated displays is a complex topic and is the subject of extensive research. Several authors have surveyed the literature and developed guidelines useful for design and evaluation of computer generated displays (Smith and Mosier, 1986; Mayhew, 1992; Sanders and McCormick, 1994; Helander, 1994; Shneiderman, 1992; Galitz, 1993). The guidelines presented come from these sources. The material is divided into three areas: general issues, hardware 
issues, and software issues.

\section{General}

\section{Hardware}

Although the locomotive cab is more spacious than an aircraft cockpit or a motor vehicle, the designer is faced with the same human constraints that limit the amount of usable space within the cab to display information. Just as the engineer is limited in how far he or she can reach a particular control by the length of his or her arms and legs, so too is the ability to see displays limited by the engineer's visual system. The size and area of the central and peripheral vision limit how far the engineer can see and the accuracy with which information will be seen (Sanders and McCormick, 1994). Current recommendations (NASASTD-300, 1994) suggest a nominal viewing distance of 20 inches $(510 \mathrm{~mm})$ and all areas of the display be viewable from within $30^{\circ}$ of the horizontal axis centered on the screen.

Currently, the amount of information that needs to be continually displayed is greater than the amount of available space within which the engineer can see. This lack of space requires the designer to make trade-offs to keep the engineer informed while minimizing clutter .

These choices are made through the selection and implementation of a particular dialog design, the use of automation and how much information is placed on the display, where it is located, and how it is coded. The goal of the designer is to present only the information that is needed in an operationally suitable form and to facilitate quick information access to minimize head-down time.

These issues include: the trade-off between the need for information and minimizing clutter so that the information can be accessed quickly and easily; and the modality by which information is displayed: visual or auditory; graphic or text . The choice of display hardware can affect image quality. The poorer the image quality the longer it takes the operator to extract information. People read information on electronic displays more slowly than on paper because the image quality is poorer on electronic displays (Sanders and McCormick, 1994). Some of the significant factors that affect image quality include: resolution, luminance and contrast, flicker and glare control (Snyder, 1994 in Tullis, Grandjean, 1987 in Salvendy, ANSI/HFS 100; NASA-STD-300, 1994) are discussed below.

Resolution. Resolution represents the smallest level of detail that a display shows. This is a function of target to background 
contrast and is expressed in terms of Modulation Transfer Function. The ANSI standard for VDTs recommends a MFTA (Modulation Transfer Function Area) of 5 (ANSI/HFS1001988). However, the standard was developed for an office environment that differs from that found in a locomotive. The recommended resolution for a given display will vary as a function of a number of variables including: viewing distance, ambient light level, task requirements and screen content (Snyder, 1994). Using a different method, NASA recommends a minimum resolution of 67 lines/inch for aerospace applications (NASASTD-3000, 1994). Resolution can also be specified in terms of the number of dots/inch needed to display a character. A minimum recommendation is a $5 \times 7$ dot matrix, with $7 \times 9$ preferred. For environments in which symbols will be rotated, the minimum dot matrix is $8 \times 11$, with $15 \times 21$ preferred.

Luminance and contrast. Objects on the display are detected by observing the differences in luminance (brightness) between objects and the background. To adequately see both foreground and background, it is recommended that the luminance of the foreground or background be at least $35 \mathrm{~cd} / \mathrm{m}^{2}$. The display should be capable of a minimum contrast ratio of $3: 1$ with $7: 1$ preferred.

The locomotive cab environment varies greatly in the level of ambient illumination. To help the engineer adapt to changes in illumination provide controls to adjust the level of brightness and contrast.

Flicker. The screen will appear to flicker when the refresh rate of the screen is too low and image persistence is too short. Flicker contributes to annoyance and user unhappiness with the display (Snyder, 1994). The refresh rate for a display should be at least 60 cycles per second for a screen with a dark background and light foreground (positive polarity). The refresh rate for a display should be at least 90 cycles per second for a screen with a light background and dark foreground (negative polarity). If the designer follows this recommendation, the image will always appear steady.

Glare. The engineer may experience two types of glare: disability glare and discomfort glare. Disability glare interferes with the engineer's ability to acquire information from the display. Discomfort glare results in viewer discomfort, but the engineer can still obtain information from the display. Over time discomfort glare and disability glare can contribute to headaches 
and fatigue. With windows on three sides of the locomotive cab, glare due to sunlight poses a significant problem.

The optimal solution to glare is to eliminate it. Surfaces should be painted in a dark color with matte finish. Light from bright windows can be shaded. The display can be oriented to avoid reflections and a hood can be used to shield the display. When glare can not be removed anti-glare treatments may be necessary. Effective anti-glare treatments have two properties (Snyder, 1994). They disperse specular glare, eliminating the image of the glare source as the engineer views the display and they increase contrast. Anti-glare treatments may include the use of neutral density filters and etched display surfaces. To accommodate the range of ambient light conditions found in the locomotive cab, provide user selectable controls to vary the brightness, contrast and color of the display. Removing the source of the glare is preferable to anti-glare treatments because anti-glare treatments may degrade image quality. Flat screen displays also reduce the number of glare sources reflected in the display.

Dark adaptation. To preserve dark adaptation, for night operation, the display should be designed to be used at the dimmest setting possible while still maintaining good image quality.

Maintenance. Displays that use incandescent lighting should incorporate dual lamps. When one bulb fails, the light intensity will diminish to indicate the need for replacement without degrading engineer performance. Bulbs should be replaceable from the front panel without the use of tools.

Software

The goal of a well designed information display is to enable the engineer to quickly and easily find and interpret the task related information to support train control. The dialog design is one part of the overall framework that controls how information is organized. Another critical aspect concerns how the information is laid out on the display. The format or coding of specific information elements and how different information elements relate to each other determine the effectiveness with which engineers can identify and extract needed information.

This section discusses the human factors issues for evaluating the display format. The topic of display design has been the subject of numerous books and publications. The following recommendations (section 5.7.3) represent a selected subset representing some of the most essential ideas. The guidelines 


\subsubsection{Recommendations}

were compiled from the following sources addressing the design of computer generated displays: Brown, 1989; Galitz, 1989; Sanders and McCormick, 1994; Shneiderman, 1992; Smith and Mosier, 1986; Tullis, 1994; and Widdell and Post, 1992.

Evaluation of the display format needs to be concerned with three aspects: what information is included, where the information is placed, and how it is structured (Galitz, 1989). How these three topics are addressed will affect the legibility and comprehensibility of the display as well as the speed with which engineers located needed information.

The amount of information should be minimized by only displaying what is necessary to the user (Tullis, 1994). Information density generally should not exceed $25-30 \%$ of active screen area (Galitz, 1994). As density of information increases, time and errors in performing the task increase.

Tullis (1994) distinguishes between local density and overall density. Local density refers to the number of characters within a confined region and measures how tightly packed the screen is. Overall density refers to the amount of information on the entire screen. Overall density may be higher if it has reduced local density. The need to minimize information should not occur at the expense of clarity or task performance. Displays that stay within density guidelines, but are incomprehensible and lack necessary information, are not preferable to more informationally dense displays that are comprehensible and contain all the necessary information.

Density can be minimized by using the following methods:

Concise wording. Use short simple sentences with a wording that the engineers will understand.

Abbreviations. Use complete words where possible. Where abbreviations save needed space, a consistent method should be used. The choice of abbreviations should be made on the basis of what engineers are likely to understand. Frequently used abbreviations schemes include: truncation, alphabetical, and abbreviations commonly recognized by the user population.

Avoid unnecessary detail. Give engineers only as much detail as they need. For example, if the engineer needs a temperature 
reading at a level of accuracy that only requires whole numbers, do not display temperature out to one decimal place, even though the system is capable of displaying this level of accuracy.

Tabular formats with column headings. Use white space to separate information into columns with a heading. Column headings save space by reducing the number of times that related items are repeated.

\section{Information Placement}



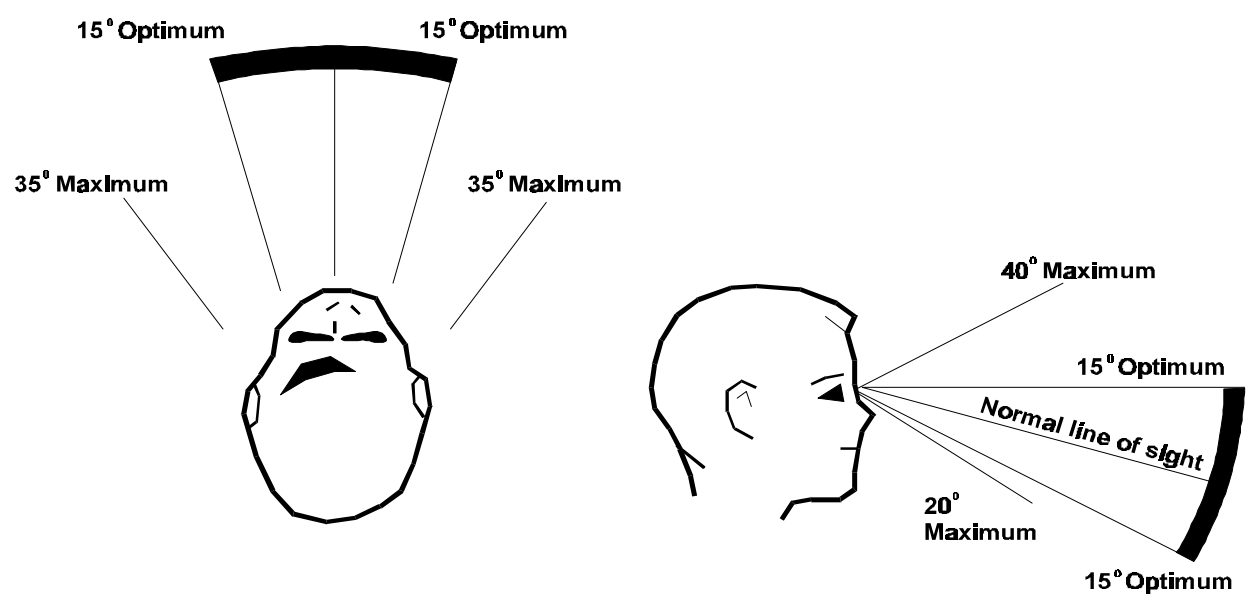

Figure 5-11. Area of Best Visual Acuity. (From Human Engineering Design Criteria for Military Systems, Equipment, and Facilities (MIL-STD-1472D), Department of Defense, 1989, p. 31.)

Grouping. Group items that belong together. Tullis (1994) points out that grouping items that belong together can facilitate readability and help the user to discriminate relationships between different groups of data. Compared to narrative text, grouped text is less complex and can be searched faster (Sanders and McCormick, 1994).

Grouping can be achieved by the use of spatial proximity (using white space to separate text or graphics), visual boundaries, and highlighting. Guidelines for highlighting information are discussed in more detail in the next section.

Group size should subtend no more than 5 degrees of visual angle (i.e., 12-14 characters by 6-7 lines) to facilitate search time. Groups that meet this guideline can be scanned in one eye fixation where larger groupings take more than one fixation and increase search time (Galitz, 1993). However, legibility and comprehension should not be sacrificed to meet this guideline.

\section{Information Coding}

Techniques for highlighting information. The amount of information that can be displayed may frequently be greater than the engineer's ability to attend to it all. Highlighting is an effective method for attracting the engineer's attention to critical items. A variety of techniques can be used to code information and vary the emphasis of that information. These techniques include: reverse video, underlining, flashing, color, shape, size, and brightness. Each coding technique has advantages and disadvantages. For example, flash coding of text is good for 
attracting attention, but is difficult to read.

To maximize the effectiveness of a particular code avoid overusing too many codes. Overuse of coding techniques to highlight information can result in unacceptably "busy" visual designs. Each coding technique competes for the limited attention of the engineer reducing the overall effectiveness of each highlighting technique. Emphasis should be placed on simplicity and clarity.

Critical, abnormal or updated data should be highlighted using reverse video, shadows, brightness, or color coding (Smith and Mosier, 1986).

Flash coding. Blink rate should be between 0.1 to $5 \mathrm{~Hz}$ with 2-3 $\mathrm{Hz}$ used to generate a strong sense of urgency. No more than two levels of blinking should be used because more will be difficult for engineers to discriminate. Flash coding should be used sparingly and never for text or number that are critical or need to be read quickly. Where readability is important, flash coding can be implemented by using a separate blinking symbol placed near the information that must be read.

Luminance coding (brightness). To avoid problems with user adjustable brightness controls keep the number of brightness levels to two, bright and dim (Smith and Mosier, 1986).

Reverse video. This technique is frequently used to show a currently selected item. It can also be used to draw the user's attention to an isolated item on the screen. Leave a buffer zone on either side of characters and symbols to avoid legibility problems caused by the characters bleeding into the background.

Size coding. If size coding is used, ICAO (1993) recommends using only two widely different sizes. Size coding can be useful to distinguish labels or headings from data.

Color. Visual displays should be first designed to meet human performance criteria under monochrome conditions. Add color only if it will help the user in performing a task (Mayhew, 1992).

The display should be usable in monochrome because some users have various levels of color deficiency and will not be able to discriminate between some colors. 
The extremes of ambient illumination found in the locomotive cab can limit the choice of colors, change the appearance of the colors displayed, and can alter brightness contrast (Hopkin, 1992 in Widdel \& Post). Research has yet to determine how best to use color when ambient illumination varies widely. Therefore, provide sufficient luminance contrast (brightness) when optimal color contrast is not feasible. Color coding should be a redundant coding technique, since some engineers may have color deficiencies.

Avoid using color in areas that are not in the engineer's direct line of sight, since it is difficult to discriminate colors in peripheral vision. When color is used in the display, symbols and text should be larger than when they are displayed monochromatically.

The number of colors should be limited to no more than six, when the task requires discriminating between colors. Humans have difficulty recognizing more than this number. This is consistent with research on human short-term memory indicating that humans can remember between 5 and 9 chunks of information. Shneiderman recommends using no more than 7 colors for the entire sequence of displays and 4 colors in an individual display.

Additional recommendations for using color include the following (Marcus, 1991; Shneiderman, 1992):

- Avoid the following color combinations: red/blue, red/green, blue/yellow, and green/blue. This combination may contribute to eye strain and muscle fatigue and create vibrations, illusions of shadows and after-images.

- Color coding should be consistent with user expectations for the task (i.e., green for go and red for stop).

- Consider the use of color to format related items in a densely packed display.

\section{Text based information (Typography)}

In presenting text on electronic displays (for example, in expert systems displays), legibility, readability, and comprehensibility are key issues that need to be addressed in evaluating its usability. Legibility refers to the rapid identification of individual characters 
while readability refers the ability to recognize the form of a word or group of words. The following recommendations address the issues of legibility, readability and comprehensibility.

Character height. Readability is affected by its size. The optimal height of characters will vary with distance of the viewer from the display. Table 5-5 shows recommended heights for different luminance conditions.

Typeface. Readability is also affected by the appearance or typeface and the case used. Numerous typefaces or fonts exist that vary in how each character is displayed. Complex or ornate typefaces (i.e., script or block letters like those found in German manuscripts of the 15th century) should be avoided. (Wieringa, Moore, Barnes, 1992). Serif and sans serif fonts are typefaces which are commonly considered for electronic displays. Serif fonts include short lines at the ends of some of the lines that form the letters as shown in Figure 5-12. San serif fonts lack these lines. Research is mixed on which font is preferable. Both typefaces may work well. Serif fonts facilitate the recognition of word forms and assist in horizontal eye movements (Wieringa et al, 1992). For this reason, some guidelines recommend using serif fonts (e.g., Times Roman) for narrative text such as system messages. Where serif fonts are used, it is important that the serifs be of sufficient thickness to be legible (Marcus, 1991). San serif fonts have the advantage of reducing clutter because they lack the serif and is frequently recommended for use in graphics and labels where they do not distract the user from the other screen elements. Sans serif typefaces are also preferable when the screen resolution is inadequate to display the fine lines of the serifs (Marcus, 1991).

Table 5-5. One Set of Recommended Heights of Alphanumeric Characters at 28 inch Viewing Distance (Adapted from Sanders and McCormick, 1994, p. 107.)

Height of numerals and letters*

\begin{tabular}{|c|c|c|}
\hline & $\begin{array}{l}\text { Low Luminance } \\
\text { (down to } 0.03 \text { fL) }\end{array}$ & $\begin{array}{l}\text { High Luminance } \\
\text { (1.0 fL and above) }\end{array}$ \\
\hline Critical use, position variable & $\begin{array}{c}0.20-0.30 \mathrm{in} \\
(5.1-7.6 \mathrm{~mm})\end{array}$ & $\begin{array}{c}0.12-0.20 \mathrm{in} \\
(3.0-5.1 \mathrm{~mm})\end{array}$ \\
\hline Critical use, position fixed & $\begin{array}{l}0.15-0.30 \mathrm{in} \\
(3.8-7.5 \mathrm{~mm})\end{array}$ & $\begin{array}{l}0.10-0.20 \mathrm{in} \\
(2.5-5.1 \mathrm{~mm})\end{array}$ \\
\hline Noncritical use & $\begin{array}{c}0.05-0.20 \\
(1.27-5.1 \mathrm{~mm})\end{array}$ & $\begin{array}{c}0.05-0.20 \\
(1.72-5.1 \mathrm{~mm})\end{array}$ \\
\hline
\end{tabular}


* For other viewing distances (D), in inches, multiply table values by D/28.

Proportional typefaces which vary the width of individual characters and the spacing between characters are generally preferred to non-proportional typefaces.

Use mixed case for presenting blocks of text, such as system messages and train order. Text presented in upper case slows the reading process due to the uniformity of the word shape and takes up more screen space. Upper case is appropriate for single words or short phrases which must attract the engineer's attention. However, to be effective this technique can not be overused.

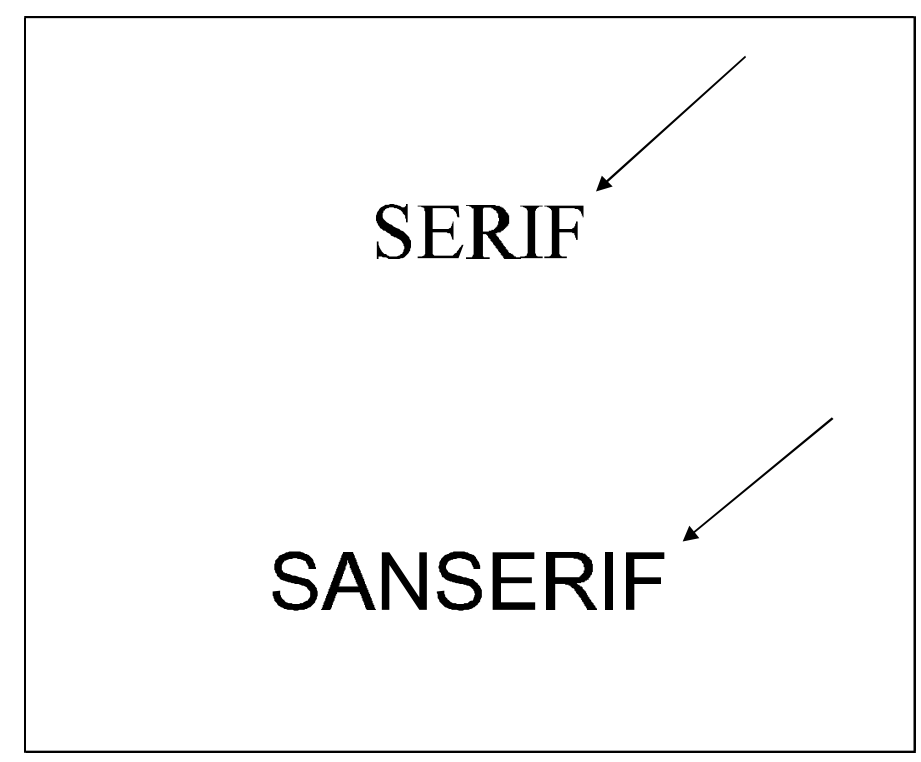

Figure 5-12. Comparison of Typefaces

White Space. Use white space to organize alphanumeric text. Cluttered displays frequently lack adequate white space. White space can help convey information structure and sequence. For intraline spacing (spacing between words), text may be left justified and right ragged, justified on both left and right (fully justified), center justified, and left ragged and right justified. The appropriate alignment will vary with the typeface, average line length and conventional usage.

For most narrative text, the designer chooses between fully justified alignment and left justified and right ragged alignment. As column width decreases, full justification becomes more difficult to read compared to left justified, right ragged text. For 
small columns, the large spaces between words slow the reading process (Marcus, 1991).

The problem of large spaces between words is exacerbated when non-proportional fonts are used. The goal should be to maintain consistent spacing between words. For most text, this will be achieved through the use of left justified, right ragged alignment.

For interline spacing, Tullis (1993) recommends that the space between the bottom of one line and the top of the characters on the line below should be equal to or slightly greater than the height of the characters themselves. This represents a ratio of letter height to total space between lines of 1:2 to 1:2.7.

Sentence structure. Where sentences will be used to communicate with the engineer the following recommendations should be considered:

Use short familiar words and a simple sentence structure. Long sentences with multiple thoughts or clauses take longer to process and are more difficult to understand. When sentences describe a sequence of events they should be described in the order in which they will occur. Sentences should also use the active voice since they are easier to understand. Express ideas in positive terms since negatively expressed ideas take longer to understand.

Wording. The engineer must understand the information on the display to be informed about the system. To help the engineer understand this information, use familiar and consistent terminology. Ensure that items are distinct from one another and

difficult to confuse with one another. Keep phrasing short and concise as possible and avoid using words that have multiple meanings.

Short words tend to be more familiar and easier to understand. However, a long familiar word is preferable to a short, unfamiliar word. While short words are generally better than long words, avoid using contractions.

When abbreviations are used, choose those abbreviation that are commonly recognized and do not abbreviate words that produce uncommon or ambiguous abbreviations. Abbreviations may reduce clutter by saving screen space. However, when too many 
abbreviations or abbreviations that are unusual are used, they may tax the engineer's memory. Whatever rule is adopted for creating abbreviations, use that rule consistently and ensure that users understand it.

Graphic information. Presenting information in a graphic format enables the designer to display dynamic information that is difficult and cumbersome to show using a text based format. Graphic displays are useful for enabling the user to quickly scan related sets of data and for monitoring conditions, like speed or location, that are constantly changing (Smith and Mosier, 1986). Graphic displays can easily show both quantitative and qualitative information as well as show whether one or more parameters are within an accepted range. Graphic information can also reduce display density when graphic displays such as symbols and icons take up less space than the equivalent representation in words.

Analog versus digital displays. Graphic displays present the designer with new opportunities to rethink the design of current gauges and dials as well as new functions. Conventional displays showing speed or brake pressure may be represented as analog format or in a digital format that changes the quality of the information displayed. Figure 5-13 shows the flexibility with which a speed may be displayed using electronically generated symbols and characters.

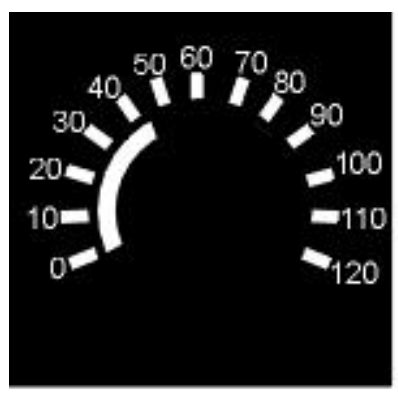

Circular

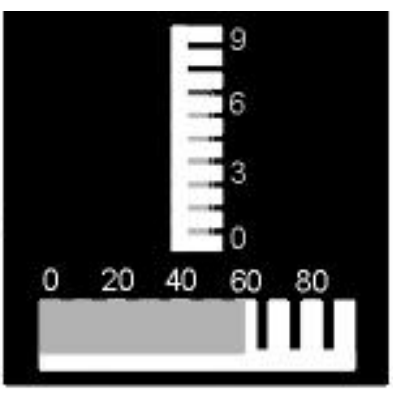

Horizonta \& verticoll

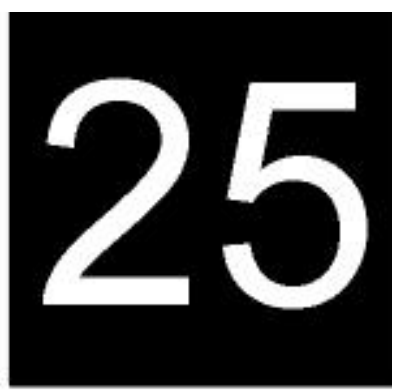

Digital

Figure 5-13. Example of Three Electronically Generated Displays Showing Speed (Adapted from Human Factors in Engineering Design, Sanders and McCormick, 1994, p. 134. Used with permission from McGraw-Hill, Inc., New York, NY.) 
The information requirements of the engineer should drive the requirements for how to display graphic information. Digital displays are generally preferred to analog displays when a precise numerical value is required; the values remain displayed long enough to be read and are not continually changing (Sanders and McCormick, 1994). Analog displays are advantageous when the values are continually changing and the direction or rate of change is important to the user (Sanders and McCormick, 1994).

For example, the digital counter shown in the right panel of Figure 5-13 makes a poor display for judging speed. As speed increases, it becomes increasingly difficult to read because the numbers change too rapidly. A curvilinear display like the one shown in the left panel of Figure 5-13 betters suits this task because the display shows range of the scale as well as the actual number. It supports behavior in which the engineer may try to keep the locomotive within a limited range rather than maintain a precise speed.

The relationship among graphical elements should convey to the user the physical or functional relationship among actual elements.

Symbol design. Coding information symbolically has a number of advantages. Just as a picture can represent a thousand words, symbols can minimize the amount of screen clutter compared to text. Symbols and icons in particular can aid recognition and comprehension by representing objects in ways that mimic their appearance (Tullis, 1994). However, to be effective, the designer must be able to create symbols and icons that are meaningful and familiar to the user. The following guidelines give recommendations for evaluating symbology (Galitz, 1992; Marcus, 1992).

- Limit the number of symbols and geometric shapes used to represent objects to between 10 and 20 .

- Make the symbol or icon as concrete as possible. The more directly the symbol conveys what the object or action represents the more likely the engineer will understand.

- Keep the symbol simple. Unnecessary embellishments add screen clutter and may confuse the user. However, symbols should not be so simple that they are confused with one another. 
- Use symbols with which the engineer is familiar. Familiarity will reduce learning time.

- Use symbols that the engineer is familiar with to reduce learning time. The shape of the symbol should be clear and unambiguous. Screen resolution should be adequate to discriminate between different symbols.

- Add a label to increase comprehension. The ability to comprehend and learn symbols can be improved by attaching a label.

- Create consistent shapes through limiting the use of angles, line thickness, shapes and amount of empty space.

- Arrange the symbols in a meaningful way. The symbols should be organized in a way that reflects the way the engineer will act on the symbols.

\subsection{COMPUTER INPUT DEVICES}

This chapter discusses the human factors issues related to the use of computer input devices in the cab. Computer input devices consist of keyboard, pointing devices such as a mouse, light pen and trackball, programmable function keys, and the touchpad. Only the devices that are most likely to find their way into the locomotive cab are discussed in detail.

\subsubsection{Operational Issues}

Environmental conditions. The ability to withstand the environmental conditions found in the cab is a driving force in designing equipment for the locomotive cab. Computer input devices must withstand the variation in temperature, humidity, vibration, electromagnetic interference, and dirt. The engineer may need to use these controls using gloves. The locomotive cab is a harsh environment compared to the typical office environment in which these devices are usually found.

The harsh environment may be one reason the ATCS specification developed for the AAR uses function keys for response selection. The vibration levels currently found in the locomotive cab make it more difficult to use pointing devices like the mouse or light pen compared to function keys or a touchpad.

Maintaining situational awareness of what is going on outside the 
locomotive $\mathrm{cab}$ is essential for the locomotive engineer. The choice of computer input device should be based on its ability to minimize head-down time. This would suggest minimizing the use of keyboard entry activities, since typing can be time consuming. Too much computer input via keyboard would give the engineer less time for other activities involving his or her hands. However, the need to maintain situation awareness outside the cab window suggests that input devices requiring less heads-down time would be more appropriate or operationally suitable.

\subsubsection{Human Factors Considerations}

Computer input devices represent an alternative to electromechanical controls for controlling the locomotive. They can perform control functions much like conventional electromechanical controls or they may be used to enter information into the system. The design, arrangement and functioning of the controls all influence the effectiveness of the system. Design dimensions such as shape, size and material must be compatible with human anatomical and physiological characteristics (Cardosi and Murphy, 1995). Other dimensions relate to the user's task needs such as the degree of accuracy, force, precision and manipulation.

Currently, there is no published research evaluating the usability of computer input devices in the locomotive environment. However, the following guidelines adapted from Cardosi and

Murphy (1995) can be applied to the evaluation of computer control devices in the locomotive cab. The following general guidelines apply to all control devices (Ivergard, 1989):

- Keep the number of controls to a minimum.

- Control movements which are natural for the operator are the best and least tiring.

- Design the controls to make enough resistance to prevent their activation by mistake.

- Design the controls to cope with misuse.

- The control should give feedback so the engineer knows when it is activated.

Several issues need to be considered in the design and use of 
input devices. The devices considered here include keyboards, touchscreens, and trackballs. While the selection of a specific type of input device will depend upon the task at hand, in no case should an overall design of input devices require frequent switching between devices. To ensure that the performance of the device matches the needs of the engineer, positioning accuracy and time lag between input and output should be taken into account when evaluating the device.

Keyboards. Standard QWERTY keyboards are still the major input devices which people are most familiar. (The term "QWERTY" comes from the arrangement of six keys to the left, above the home row [ASDF] for the left hand). The typical keyboard also includes arrow keys and function keys.

The QWERTY keyboard is sequential, with one key representing one and only one character (e.g., a number or letter), and with individual keys being activated in a sequence to produce a word or some other character string.

The QWERTY keyboard is designed for text entry. However, the locomotive cab environment is not conducive to entering text, where motion and vibration make it difficult to accurately enter text. This means that the traditional QWERTY keyboard is illsuited as a computer input device.

Good keyboard design is essential to provide accurate and timely data entry input. The design of a keyboard can make practical differences in the speed and accuracy of data entry input. In addition, use of poorly designed keyboards can lead to muscle fatigue in the arms and possible nerve damage in the wrist and fingers.

\subsubsection{Recommendations}

\section{Evaluation Guidelines for Programmable Function Keys (Soft Keys)}

AAR specifications propose the use of programmable function keys (alternatively called a soft function key menu interface). The following guidelines specifically address this type of interface (Brown, 1989; Mayhew, 1992; Smith and Mosier, 1986). Optimizing a programmable function key interface, designers need to consider a number of issues: labeling, spatial layout, and consistency.

Provide enough function keys to support functionality, but not so 
many that scanning and finding the functions are difficult. In evaluating the number of function keys, the amount of screen space and keyboard space to allocated function keys will need to be considered. There is no rule of thumb for the number of keys that are appropriate. However, as the number of keys increases beyond a certain level, the keyboard or workstation and display will become increasingly crowded, making it more time consuming to search for the relevant response selection and implement that selection.

Coding schemes. Use space, size and color to distinguish groupings. This will facilitate the search for specific keys and aid memory for the meaning of the different keys. For example, the designer could place function keys that have destructive modes (e.g., the delete key) away from the main keys to avoid accident activation. Placing keys with potentially destructive effects in hard to reach places makes it less likely the engineer will press it by mistake. Keys could also be color coded to be consistent with user expectations (i.e., green for go, red for danger or warning). Arrange keys in groups of three or four based on the screen display.

Frequency of use. Place the most frequently used keys in the most accessible positions. Within a set of function keys, some keys may be more accessible than others. For example, in a single row of keys, the keys on the end are more accessible than the keys located in the interior. Place the most frequently used keys in these positions to speed response time.

Qualifier keys. Minimize the use of qualifier keys. It is frequently difficult to develop a meaningful method for assigning key sequences using qualifier keys (i.e., Shift, Alt, Command, Control). The lack of useful memory aids for assigning key sequences increases the memory load on the engineer. Multiple key strokes are also more prone to error. However, when qualifier keys cannot be avoided, use them consistently. Consistency in logic helps to learn the functions associated with that key.

Control-display compatibility. Preserve spatial relationships between soft function key labels on the screen and generic function keys on the keyboard. Consistent relationships between the display and the control is essential to foster learning of the system, ease of use, faster performance and minimize errors. Using the same coding schemes (space, size, grouping) for both the displays and controls. 
Consistency in key assignment. Be consistent in function key assignments across screens and subsystems. Smith and Mosier (1986) recommend that when a function key performs different functions in different operational modes, the designer should assign equivalent or similar functions to the same key. For example, a particular key might confirm data changes when operating under ATCS and confirm message transmission when in a non-ATCS environment.

Feedback. The system should give the user feedback regarding the status of function key operation. When function keys are pressed, the system (i.e., BN' s ARES System) should give the operator feedback to indicate whether some action took place and how the system is responding. This may include visual, auditory and/or tactile feedback. Where key labels remain the same, some system change takes place that makes a particular menu selection inoperative, the inactive key or menu selection should be grayed out or deleted to indicate this change. If there are special keys that have different modes (e.g., Caps Lock), a status indicator should indicate what mode the keys are in. For example, a light on the function key could indicate for which mode the function key is set by whether the light is on or off.

Navigation. Enable easy access to the home menu. In hierarchical menu systems, with multiple levels, the user may need to quickly return to the home menu to initiate a new sequence of actions. If the functions assigned to a set of keys changes as a result of user selection, give the user an easy way to return to the home menu.

\section{Evaluation Guidelines for Standard Keyboards}

Extensive research on keyboard design has yielded numerous design guidelines (DoD, 1989a; DoD, 1989b; Gilmore, Gertman, and Blackman, 1989; Lueder, 1986; Smith and Mosier, 1986). Some of these guidelines are presented here:

- Height, Thickness, and Slope. Keyboard height should be adjustable by the controller within a range from 23 to 32 inches (i.e., it should be possible to raise and lower the surface that supports the keyboard). The keyboard should be less than $30 \mathrm{~mm}$. thick from its base to the home row of keys, and its slope should be adjustable between 15 and 25 degrees from the horizontal (Gilmore, Gertman, and Blackman, 
1989).

- Arrangement, Labeling, and Feedback. Alphanumeric keys should be logically arranged and must meet standards for dimensions, displacement, and separation, as provided in Figure 5-14. If extensive numeric data are to be entered, a separate numeric keypad should be provided, visually distanced from the main keyboard, and arranged in a $3 \times 3+$ 1 matrix with zero (0) centered on the lowest row. To reduce syntax error, function keys should be provided for frequently invoked commands.

Function keys should be clearly labeled to identify their functions, and these functions should be consistent throughout the system.

Requiring engineers to use keyboards with different layouts risks transfer errors of many kinds and is likely to increase the time needed for data entry. Nonactive keys should be blank; mechanical overlays should not be used to restrict access to nonactive keys. The key used to initiate a command must be clearly labeled "Enter." Keyed data should be quickly displayed or "echoed" on the screen. Tactile feedback should verify keystrokes and inform the controller when the next action may be initiated.

\begin{tabular}{|c|c|c|c|c|c|c|c|}
\hline \multirow{5}{*}{$\begin{array}{l}\text { Minimum } \\
\text { Maximum } \\
\text { Preferred }\end{array}$} & \multirow{2}{*}{\multicolumn{2}{|c|}{ DIMENSIONS* }} & \multicolumn{5}{|c|}{ RESISTANCE } \\
\hline & & & \multicolumn{2}{|c|}{ Numeric } & \multicolumn{2}{|c|}{ Alpha-numeric } & Dual Function \\
\hline & \multicolumn{2}{|c|}{$\begin{array}{c}10 \mathrm{~mm}(0.385 \mathrm{in} .) \\
19 \mathrm{~mm}(0.75 \mathrm{in.}) \\
13 \mathrm{~mm}(0.5 \mathrm{in} .)\end{array}$} & \multicolumn{2}{|c|}{$\begin{array}{c}1 \mathrm{~N}(3.5 \mathrm{oz} .) \\
4 \mathrm{~N}(14.0 \mathrm{oz} .)\end{array}$} & \multicolumn{2}{|c|}{$\begin{array}{c}250 \mathrm{mN}(0.9 \mathrm{oz} .) \\
1.5 \mathrm{~N}(5.3 \mathrm{oz} .)\end{array}$} & $\begin{array}{c}250 \mathrm{mN}(0.9 \mathrm{oz} .) \\
1.5 \mathrm{~N}(5.3 \mathrm{oz} .)\end{array}$ \\
\hline & \multicolumn{5}{|c|}{ DISPLACEMENT } & \multirow{2}{*}{\multicolumn{2}{|c|}{ SEPARATION }} \\
\hline & Numeric & & -numeric & & al function & & \\
\hline $\begin{array}{l}\text { Minimum } \\
\text { MaxImum } \\
\text { Preferred }\end{array}$ & $\begin{array}{l}0.8 \mathrm{~mm}(0.03 \mathrm{in} .) \\
4.8 \mathrm{~mm}(0.19 \mathrm{in} .)\end{array}$ & \multicolumn{2}{|c|}{$\begin{array}{l}1.3 \mathrm{~mm}(0.05 \mathrm{in} .) \\
6.3 \mathrm{~mm}(0.25 \mathrm{in} .)\end{array}$} & \multicolumn{2}{|c|}{$\begin{array}{l}0.8 \mathrm{~mm}(0.03 \mathrm{in} .) \\
4.8 \mathrm{~mm}(0.19 \mathrm{in.})\end{array}$} & \multicolumn{2}{|c|}{$6.4 \mathrm{~mm}$ (0.25 in.) } \\
\hline
\end{tabular}

*Refers to dimension D shown below.

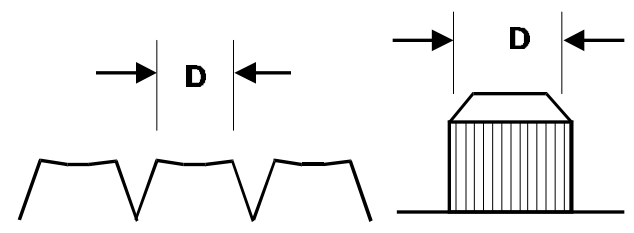

Figure 5-14. Keyboard Dimensions, Resistance, and Displacement (From Human Engineering Design Criteria for Military Systems, Equipment, and Facilities by the Department of Defense, 1989, Table X, p. 95.) 
- Location. The keyboard should be located a comfortable distance from the controller and directly in front of and below the associated visual display. The keyboard slope should be adjustable between 15 and 25 degrees from the horizontal. Forearm and wrist support should be provided to reduce discomfort.

- Other Considerations. Keyboards must be readable under all operating conditions. Guards should be considered for any key that would present a problem if inadvertently activated.

Detailed requirements for keyboards are given in MIL-STD-280 (DOD, 1989a).

Split keyboards have recently been developed to reduce the strain on users' wrists. An even more recent development is the halfQWERTY keyboard, which permits one-handed typing on a keyboard half the size of the standard keyboard (Matias, MacKenzie, and Buxton, 1993). It is not known whether these keyboards are suitable for the locomotive cab environment. To avoid increasing engineer workload, it is important to minimize requirements for keyed data entry (DoD, 1989).

Touchscreens. A touchscreen device generates an input signal in response to a touch or movement of the finger on the display. Typically, touchscreen devices operate by producing $\mathrm{X}$ and $\mathrm{Y}$ position data whenever a touch is detected by the system. The most common version has two conductive layers, each with an electrode grid in both the $\mathrm{X}$ and $\mathrm{Y}$ directions. When pressure is applied, the two surfaces touch, and the circuit is completed.

Touch-sensitive screens may be useful where space is at a premium and where use of a keyboard is not recommended (e.g., where head-down time must be minimized).

An advantage of a touchscreen is that the relationship between the user's input and displayed output is straightforward.

Several strategies allow a user to select a target or menu item on a touchscreen. Using the land-on strategy, the user selects an item by touching its location on the screen or landing on the item. Using the first-contact strategy, the user drags a finger to a selectable item, which is selected on contact. The lift-off strategy allows the user to drag a cursor onto the intended target, which becomes selected when the finger is lifted off the touchscreen. 
All of these strategies are problematic in the railroad environment. The best selection strategy for engineers will depend upon the task and the conditions under which the task is to be performed. Minimal training, limited error possibilities, and the availability of valid options only are among the advantages typically attributed to touchscreens.

Evaluation Guidelines for Touchscreens. Touchscreens have significant drawbacks. They are at a particular disadvantage in dirty environments. They are difficult to use in a moving vehicle because they do not provide tactile feedback. The operator must usually position his or her finger before making a command. Other issues include:

- Device suitability. For a task involving considerable keyboard input, a touchscreen may be inappropriate due to the requirement to remove a hand from the keyboard home row position. Frequent switching between input devices can lead to slower task completion times and/or increased error.

While touchscreens are suitable tools for form-filling dialogues or menu selections, they may be less suitable for other dialogue types such as command entry.

- Physical characteristics and layout. The sensitive areas of touchscreens should be large enough to permit activation by fingers if the land-on strategy is being used.

- Input strategy. For land-on strategies, the software accepts the touch immediately, preventing the user from verifying the correctness of the spot before activation (Shneiderman, 1992). Lift-off strategies allow the user to touch the surface, drag the cursor to the desired location, and lift off after verifying the correctness of the spot before activation. The appropriate activation strategy should be determined in the context of the engineer's tasks.

Finally, touchscreens should not be used if the task will require engineers to keep their arms up and unsupported for lengthy periods (Gilmore, Gertman, and Blackman, 1989).

Trackballs. Ball controls called trackballs or rollballs can be used for positioning the cursor and selecting data. A typical trackball design is shown in Figure 5-15. Research conducted in the area of battlefield automated systems (Parish, Gates, Munger, Grimma, and Smith, 1982) indicated that trackballs are excellent for designating and moving symbols on a display because they 
are fast and accurate. Cleanliness is also a major factor in the use of trackballs as input devices. Dirt transferred from the hands or gloves of the train crew or liquid spills can seriously impair trackball functions.

Trackball design will have to address the cleanliness issue if it is to function effectively in the locomotive cab. Vibration from the movement of the locomotive is another area for concern. Excessive vibration may increase the time it takes to select objects with the trackball. However, new locomotive cab designs that isolate the cab from other parts of the locomotive minimize the effects of vibration. There are also times when the locomotive is not moving or motion is minimal. One example, where the trackball may be an effective computer input device, is for use by the conductor managing information regarding the train consist. When trackballs are used, the following other issues should be considered:

- Limb Support. Support should be provided for the controller's wrist or arm when the trackball is used for precise or continuous movement (DOD, 1989b).

- Dimensions, Resistance, and Clearance. Physical dimensions, resistance, and clearance on the work surface should meet the criteria given in Figure 5-14 from MIL-STD1472D (DOD, 1989b). 


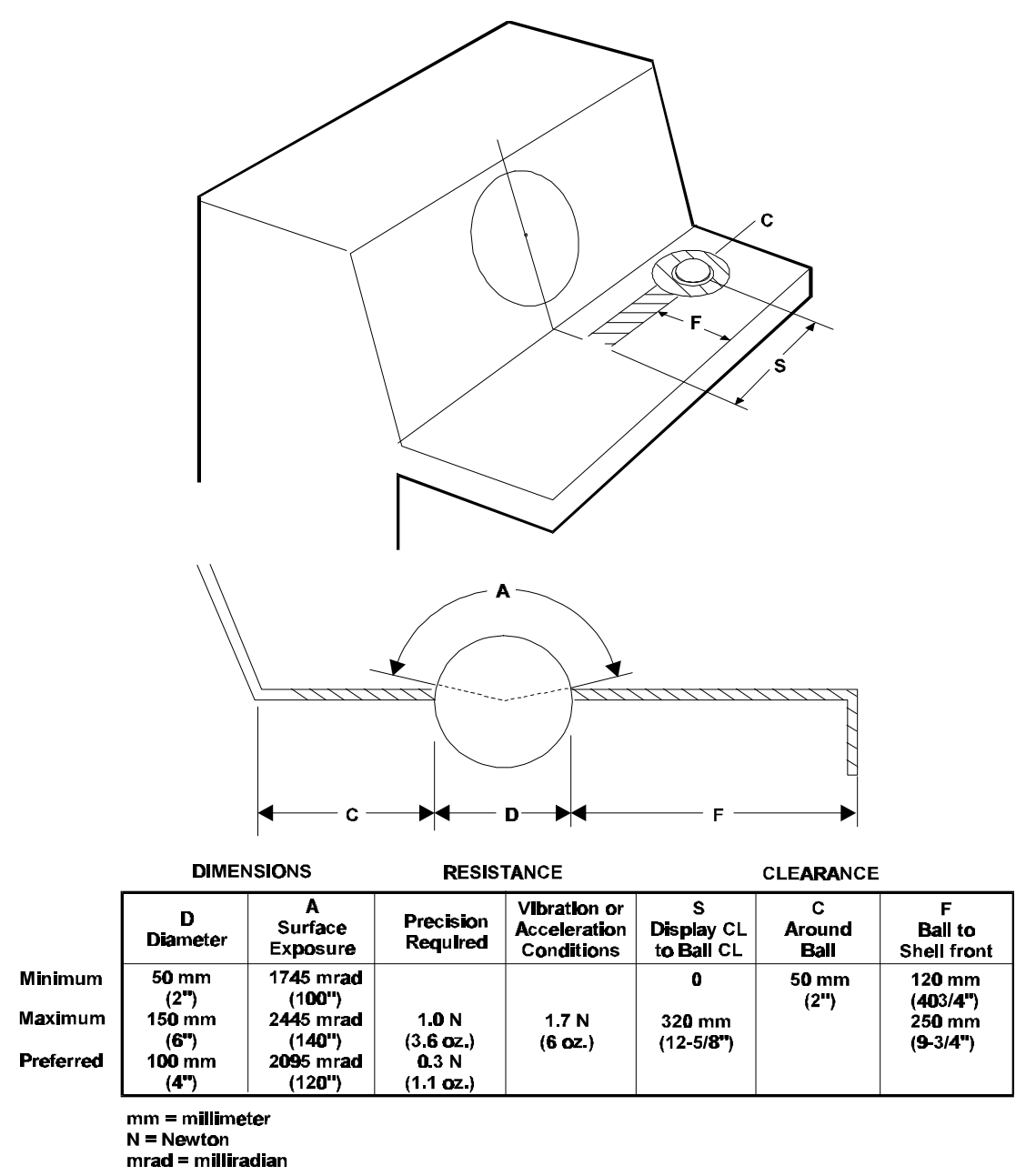

Figure 5-15. Trackball Design (From Human Engineering Design Criteria for Military Systems, Equipment, and Facilities (MIL-STD-1472D), Department of Defense, 1989.)

In addition, a discrete mechanism should be provided to activate and deactivate the device. In general, smaller trackballs should only be used where space is at a premium and precise positioning is not required (DOD, 1989b).

Central issues with respect to trackballs are the handedness of the user (i.e., left- or right-handed) and the relationship between trackball movement and movement of the cursor on the screen. This relationship should be consistent, so that the controller will be able to predict cursor movement based on force exerted on the trackball. Precise cursor positioning depends on a predictable relationship between cursor and trackball control.

Evaluation Guidelines for Trackballs. The following human factors guidelines should be met by the design of any trackball: - Dynamic Characteristics. The trackball should be able to 
move the cursor in any direction without displaying any cross-coupling (i.e., cursor movement in the opposite direction). Cursor control ratios should permit both rapid gross positioning and smooth, precise fine positioning. When it is possible to drive the cursor off the edge of the display, indicators should be provided to advise the controller how to drive the cursor back onto the display (DOD, 1989b).

\subsection{VOICE COMMUNICATIONS}

\subsubsection{Human Factors Considerations}

As described in Section 3.4, current noise levels found in the locomotive cabs can interfere with voice communications. Federal regulations allow noise levels in the locomotive cab that may impede voice communications. Federal regulations allow limited continuous noise to $90 \mathrm{dBA}$ for 8 hours. Speech intelligibility is more sensitive to noise level than hearing loss. Military standards (1472D) recommend a $75 \mathrm{dBA}$ limit to ambient noise levels for areas requiring occasional telephone use or direct communication at up to 5 feet. The equipment for producing, transmitting and displaying voice communications must be capable of providing the listener with speech that is intelligible in the relatively noisy environment found in many cabs, today. If hearing protection is required, the speech input and output devices should incorporate this feature.

\subsubsection{Recommendations}

Two issues, intelligibility and ambient noise level will affect the voice communications. Human speech occurs within a frequency range between 200 and $6000 \mathrm{~Hz}$. For this reason, human factors guidelines (DOD, 1989; NASA, 1989) recommend that microphones, speech transmission hardware, and output devices like headphones and speakers accommodate this range of frequencies. When system requirements constrain this requirement, the minimum acceptable frequency range should fall between 250 and $4000 \mathrm{~Hz}$. Microphones should have a dynamic range wide enough to pick up variations of at least $50 \mathrm{~dB}$ in signal input (Cardosi \& Murphy, 1995; DOD, 1989; NASA, 1989).

Speech transmission devices should minimize the effects of noise. This may include increasing the level of the signal in relation to the noise and clipping the peaks of the speech signal. Peak clipping refers to cutting off extremes in amplitude of the speech signal. Peak clipping has the effect of increasing the intensity of the consonants in relation to the vowels, which are 
more susceptible to interference. The effects of noise can also be minimized by preventing noise from reaching the microphone.

When speech input is also channeled through the speaker' $\mathrm{s}$ headphones, the design should preclude squeal problems and echo effects by isolating feedback between the headphones and the microphone. Reproduction of the speaker' s communication in the headphones should be in phase with the actual input to avoid an out-of-phase side tone.

Binaural headphones should be used where the ambient noise level is $85 \mathrm{~dB}(\mathrm{~A})$ or above. Binaural headphones should be wired so that sounds reach the two ears in opposing phases, unless operation requirements mandate otherwise (DOD, 1989; NASA, 1989). When headphones are used, it also recommended audio input from the sources in the cab (i.e., alarms) be routed to the headphones as well as externally (Cardosi and Murphy, 1995). Where multiple audio sources may displayed, overlapping presentation should be avoided.

The train crew should have control over the loudness level of speech messages. Volume controls should prevent the train crew from reducing the loudness level to an inaudible level. The volume control should be able to produce an overall sound pressure level of $100 \mathrm{~dB}$ (DOD, 1989). Volume controls should be separate from power controls.

Continuously monitored communication channels should include a signal-activated switching device (squelch control that suppresses noise (static) during inactive periods. The train crew should be able to manually deactivate the squelch using an on-off switch when weak signals are detected (Cardosi and Murphy, 1995).

Headsets and speakers should be designed to avoid sudden shifts in sound levels. Sudden changes in strength of the signal, from a weak signal to a strong signal can impair the operator' $\mathrm{s}$ hearing as well as create anxiety (Burlington Northern, 1989). Constant level amplifiers can protect the operator from these problems by modulating the incoming signals to a predefined signal level. 



\section{CHAPTER 6. FUTURE DESIGN CONSIDERATIONS}

The pace of change in locomotive cabs has increased in recent years. The American Wide Body Cab introduced the control console and other cab changes in the late 80s. Locomotives developed in the early 90s added electronic displays and AC traction motors. This contrasts with earlier locomotive designs that remained fairly static for decades. This chapter explores some areas that may experience human factors improvements by adding to current cab design with existing or feasible technology.

Automation using computer technology is changing what the engineer may do on the job. Initial changes may represent lower levels of automation than concepts like the Advanced Train Control System (e.g., cruise control). Automation can be used to execute the detailed steps in a task and shift the engineer's role to giving orders to the locomotive and judging the effects of the control strategy being used. For example, modern fighter planes are designed to be inherently unstable and use computers to constantly adjust trim to provide stable flight. This results in greater performance than if human-only control were used. The pilot still directs the plane, but the constant adjustments (and high workload) needed to maintain the flight path are performed by the control system. The pilot is freed to judge whether the maneuver meets the immediate situational needs. The possibility of a pilot error that may compromise stable flight is also eliminated without creating a perception that the pilot is no longer flying the aircraft. Traction control and yaw control systems on automobiles is another example of this level of automation.

The example enhancements given in this chapter are possible changes that could occur in the near term and serve as concrete examples of ways to use the automation guidelines from Chapter 5 to evaluate human factors ramifications. The emphasis in this chapter will be on how the engineer's tasks can become more user centered.

The operation of a freight train is a complex process that differs each time in many variables that affect performance. Train length, train make up, weight distribution, power availability, etc., all can change handling characteristics greatly. This makes the task too complex for current technology to perform autonomously. Therefore, the engineer as passive monitor role is not an appropriate design goal. Based on this premise, 
automated aids should actively involve the engineer in setting operational strategy and free the engineer from detailed functions of executing it. For example, a cruise control in which the engineer sets the speed (consistent with signals and other conditions) which the control system maintains is preferable to an automatic speed control system which the engineer only monitors for conditions that require an override.

Total automation which places the engineer in the monitor role may present another problem. The mental skills in perception, planning, and control assessment needed to operate a train require practice to gain and refine. Automation may prevent the development of these skills or allow them to degrade. This could make it harder for the engineer to recognize an impending problem or to intervene with the proper actions to correct the problem. The situational awareness and alertness (a current problem area) would also tend to be lower if the engineer is solely a monitor and makes control actions as the rare exception.

\subsection{VIGILANCE MONITORING}

\subsubsection{Operational Issues} underload, stress of meeting time demands, stressful cab conditions, and periods of physical inactivity along with the physiological disruptions caused by shift work and long duty hours. To combat the lack of alertness that can result, multiple crew members provide stimulation and devices have been added to measure engineer activity and apply the brakes if none is detected. Devices used to date have been based on physical activity as an indicator of vigilance.

While vigilance devices have evolved from constant pressure foot pedals to intermittent action reset devices, they are still based on use of physical activity to indicate vigilance. The most recent design uses a button (or toggle lever) reset which delays an alarm that, if not responded to with a reset, will trigger a penalty braking. Use of controls, and even touching window frames, can also reset the alarm, which allows control actions to serve as implicit signals of vigilance. However, not all rail properties use this latter feature. These symptoms have the function of ensuring that the train does not continue to operate when the crew is asleep or incapacitated. They do not, in and of themselves, make the operator more alert. 
Multiple person cab crews can provide a distraction as well as an arousal factor. The practice of calling out signals and general activity provides a stimulus for the engineer. However, there is also the potential for conversations to distract the engineer and decrease vigilance. The Federal Aviation Administration instituted a "sterile cockpit" rule to prevent nonflight related conversations in the cockpit when less than 10,000 feet above the ground because of crashes where conversations created key distractions which lead to handling errors. The trend to reduce cab crews and the potential for single person operation also reduces this method's usefulness as a means to enhance vigilance.

\subsubsection{Human Factors Considerations}

Wakefulness versus Attention

Table 6-1. Possible Vigilance Device States to the job.
The use of devices to detect physical activity determines if the engineer is awake. However, true vigilance requires mental attention to the task. Wakefulness is a necessary, but not sufficient, condition for ensuring vigilance. Current vigilance devices set up four possible conditions (see Table 6-1). First, the device can detect activity and the engineer's attention is on the task. Second, the device does not detect activity, but the engineer is attending to the task. Third, the device can detect activity, but the engineer is not attending to the task. Fourth, the device does not detect activity and the engineer is not attending

\begin{tabular}{ccc}
\hline Condition Number & Activity Detected & Attention on Tasks \\
\hline 1 & Yes & Yes \\
2 & No & Yes \\
3 & Yes & No \\
4 & No & No \\
\hline
\end{tabular}

The first and fourth states represent proper operation as a vigilance device. The second and third states indicate a flaw in this type of device. The second state is more of an annoyance that can be corrected by a reset action. However, this may distract from the real task. This is more likely in systems that do not use control actions for a reset or represent a pure secondary task (unrelated to train control--e.g., holding a deadman pedal). The third condition is a false positive that can become a safety problem. These latter two states highlight the potential for 
improvement in vigilance devices that depend only on physical actions.

Task Support

\subsubsection{Recommendations} relate to the task of operating the locomotive (except in the sense of avoiding an alarm and penalty braking). This makes it a secondary task and one that is performed mainly to avoid the irritation of the alarm and the disruption of a penalty braking.

Brandon (1979) describes the engineer's task as primarily mental; comprising the assessment of current operating conditions and planning the control actions needed to meet future requirements. A vigilance system that supported the planning function would have the advantages of being task related and ensuring that the engineer's attention is on the task.

Wilde and Stinson (1978) propose a system design of this type. They call it the Device for Attention Monitoring and Excitation (DAME). It displays the signal aspect for the next block to the engineer and requires the engineer to acknowledge the specific aspect. If the acknowledgment is correct, an OK light is lit and stays lit until the next block is entered or the original aspect changes. An acknowledgment error or failure to acknowledge within a fixed time produces an alarm (visual and aural) and requires a correct acknowledgment within a fixed time to avoid a penalty braking. A second error or error after the first grace period expires without an entry results in a penalty braking. The engineer is cued to acknowledge the next signal by a possible change in displayed signal for the next block, the OK light going out, and the alarm going off, if the grace period is exceeded.

This system is based on the engineer's accurate recognition of upcoming signals. Attention to the signal indicator is required, information of future conditions is communicated, and accuracy of perception is measured. Wilde and Stinson (1978) cite a 1972 study of railroad accidents by Kurtz which attributed 19.8\% (44 of 222) to failure to obey signals. Devoe and Abernathy (1977) conducted experiments which found that response errors were significantly higher when signals were different from what was expected. Signal expectations may develop from the practice of engineers operating on the same routes. A vigilance system of this type not only requires wakefulness, but it demands jobrelated attention and provides feedback on information receipt that has distinct safety implications. While a signal still may not be obeyed, the engineer will only violate it knowingly. 
The vigilance systems currently in use stress the physical aspects of the engineer's task. Brandon (1979), Hale and Jacobs (1975), Robinson et al. 1976), and the McDonnell Douglas Corporation (1972) studies of locomotive operation stress the mental and perceptual aspects that predominate the job. A vigilance system related to these mental requirements would match the job's attention needs better and could provide additional benefits. The potential for the engineer to make entries which reflect perception of operational conditions can provide an error correction mechanism that may yield increased safety.

The DAME device proposed by Wilde and Stinson (1978) is an example of characteristics that an improved vigilance system could possess. Devoe and Abernathy (1977) also discuss the desirability of a similar signal matching system as the basis of an alerter. The ultimate system could include other known event triggers, such as whistle points and restricted speed zones, to add to signal indications as action requirements to be monitored. This would increase the information available to the engineer to plan control activities and ensure that the engineer is correctly aware of the upcoming conditions. The use of control actions should also be accounted for in this type of system to avoid interference with ongoing tasks.

6.2 CONTROL-BY-WIRE

\subsubsection{Operational Issues}

Control-by-wire is used extensively by the aircraft industry. The traditional hydraulic and electromechanical links used to connect cockpit controls with their respective mechanisms have been replaced by wires which carry digital signals. One of the advantages is that a microprocessor and status sensors can be placed in the system to add new control capabilities. The SD70MAC represents a first step in this direction. The braking system is microprocessor controlled and the Integrated $\mathrm{Cab}$ Electronics (ICE) performs some signaling and diagnostic checks.

The large majority of locomotives use pneumatic and electromechanical control links. This has two ramifications for the engineer. First, the control mechanisms that the engineer uses have mechanical size constraints that affect placement and space available for other controls or displays. This can cause less than optimal placement. Second, the responsibility for performing all steps required by the system must be borne by the engineer. This can lead to errors, particularly for infrequent procedures, or the possibility of taking actions that will cause damage. Mechanical interlocks are often installed on controls to 
prevent these incompatible actions.

The complexity of these mechanical controls makes repairs more frequent and involved than a control-by-wire system would require. This raises the costs of maintenance as well as the chance of failure while the locomotive is in service.

The SD-70MAC represents an example of a step towards true control-by-wire for locomotives. The braking system is under full microprocessor control and engine control is performed using a computer interface. There are some instances where the system could add some assistance and reduce human actions which could lower workload and potential for error. An example of this is a maintenance procedure to discharge the DC link filter capacitors. The procedure requires the person to verify on the ICE that both inverters (TCC1 \& TCC2) are cut in before moving a switch to the isolate position. A menu selection to "discharge grids" which automatically cut in both grids would be better. This situation is worsened by the fact that there is no positive indication that the grids have been discharged (another desirable automation feature). If one of the grids is not cut in and the maintainer believes that the discharge has been done correctly, a serious safety problem may occur.

\subsubsection{Human Factors Considerations}

Traditional control mechanisms put the full procedural burden on the engineer. This reliance on human capability can introduce limits that constrain system performance. From a safety perspective, it creates greater responsibility for avoiding errors on the engineer, except for mechanical interlocks which prevent specific actions. This workload distracts the driver from the more mental aspects of controlling the locomotive to meet an ideal fit for the conditions. The greater need to concentrate on the mechanics of control actions is one reason for a new engineer's lower facility to handle a train consist.

Full control-by-wire automation with microprocessor intervention can permit the engineer to enter a command and the system to bear the burden of executing the detailed steps involved. The result is that the engineer can devote more effort to the higher level tasks of control strategy and assessment and less to mechanical tasks imposed by the system. This level of

automation provides convenience for frequent tasks and error prevention for infrequent tasks. 
The SD-70MAC does not achieve this level of automation. The brake computer automatically arranges valve settings when selecting operating ends and only requires the engineer to make one entry, the operating direction. In contrast, when operating with trailing DC units, the engineer is required to pause at the idle setting for ten seconds when going from throttle to dynamic braking to avoid a surge in braking effort (and possible DC motor damage). This delay is not needed for AC traction-only consists. A control system which had consist characteristics entered at the beginning of a run and performed the delay automatically would eliminate this potential for unexpected brake action and relieve the engineer of possible conflicts with habit (negative transfer). Also, unlike the DC traction motors, AC traction motors cannot be reversed (plugged) to add braking effort in an emergency situation. The computer does not provide any notice to the engineer that this action (a reaction that may be ingrained from long DC experience) will have no effect.

With the differences in certain operating procedures between locomotives with AC and DC traction motors and the likelihood of both types of locomotives mixing for a long time, a control system that automatically compensated for operational differences would unburden the engineer from remembering several control techniques based on consist makeup.

\subsubsection{Recommendations}

Brandon (1979) characterizes train operation as a slow-action, long-lag skill that requires anticipation of future conditions. This skill is mostly internal, developed by experience, and stored as an enactive (physical action) representation of the process. An automation system that relegates the engineer to the role of a system monitor will prevent development of this type of skill or encourage the skilled engineer to maintain a high enough level of awareness to take over in case of system failure. If the engineer does not have a clear mental picture of how the train should be performing now to meet a future time and/or speed goals, he cannot judge if the current operation is acceptable. Since control-by-wire will form the backbone of system automation, it is important that it reinforces the role of engineer as train controller and not reduce the engineer to a monitoring role. Brandon (1979) suggests that aids for the engineer should serve one or more of four purposes:

1. To improve the sense of present position. Meaningful and reliable location cues that represent decision or action points help 
the engineer assess previous actions or future events.

2. To help predict results of control actions. The use of quickened or predictive displays which will give the engineer information about the effects of control actions can help meet time and/or speed targets.

3. To maintain a high level of motivation. The ability for an engineer to explore the limits of personal skill in controlling the locomotive is a strong motivating force and source of job satisfaction.

4. To provide the visual, acoustic, and kinesthetic cues to increase the varied sources of information to the engineer. Brandon notes a desire of engineers "to feel at one with the train" and automation can create a lack of the aspects that give an engineer varied and immediate feedback of current state or control actions.

Control-by-wire is most likely to affect the ability of the engineer to exercise control skills. However, as automation sophistication grows, the entire set of perceptual and mental skills can be affected. These automated aids need to be evaluated at both the detail level (e.g., workload effects) and this larger role context level to determine desirability. The removal of the means or need for the engineer to exercise and hone control skills may lead to their loss. This negates the ability of the engineer to monitor system performance of a highly automated train or to assume control, in case of failure or unexpected conditions.

\subsection{POSITIVE TRAIN CONTROL}

Positive Train Control (PTC) refers to a group of technologies designed to improve the safety and productivity of train operations. With respect to safety, PTC refers to a set of safety objectives rather than a specific technology. These objectives should prevent train-to-train collisions, enforce speed restrictions, including civil engineering restrictions and temporary slow orders, and provide protection for roadway workers and their equipment operating under specific authorities. The key components of a PTC system include technology for precisely locating trains in the rail network and a digitally based communications system for passing information between different parts of the system. From a safety perspective, PTC will reduce the probability of a collision between trains or an Overspeed derailment by ensuring an accurate safe operating envelope and 
by reducing the reliance upon on a single decision maker for adequate train separation determination. Decisions made by the locomotive engineer or the dispatcher can be aided and monitored the PTC system with automatic interventions if safe operating limits are exceeded. Collisions and derailments may still occur, however, a more complex chain of events is required. The extent to which collisions and derailments may occur will be determined by the actual PTC design.

A PTC system includes the following key elements: computer aided dispatch system, locomotive, wayside equipment and nonlocomotive entities such as maintenance of way crews and equipment. The digital communication system links these elements together. Knowledge of the precise location of trains, maintenance of way crews and equipment, wayside equipment as well as their operating status gives the dispatcher and train crew better information to make more informed decisions about train movements. The AAR's Advanced Train Control Systems (ATCS) specifications and Burlington Northern's (BN) Advanced Railroad Electronic System (ARES) are two versions of a PTC system, developed in the 1980's. As first proposed, ATCS would use ground-based transponders to track train movements. By contrast, ARES, a system that was built and demonstrated on BN's Iron Range, used the Global Positioning System (satellite-based navigation system) to track train movements. Both of these systems would create an enormous amount of information about the status of the various components and which must be communicated to other parts of the system. In both designs, computer software manages the flow of information between the train and the dispatcher center as well as the other components of the system. The way in which the automation of this information flow is managed and how the locomotive engineer interacts with this new system will affect operator performance. Changing the communications system from an analog, voice-based system, to a digital, computer-based system will also change how the locomotive engineer performs his task and the kinds of errors he or she makes.

\subsubsection{Operational Issues}

One of the benefits proposed for PTC is that automation in the locomotive cab (and in the dispatch center) would mitigate the effect of operator errors on train control and dramatically lower the probability of an accident. Automation is proposed to mitigate errors in several ways. First, the automation would detect errors such as overspeeds and exceeding movement authorities and inform the engineer when these situations arise. 
Second, the automation may suggest appropriate corrective actions. Finally, the automation can also take control actions, if necessary. If the operator fails to take corrective action, the automation would override the current throttle or brake settings and eliminate the condition that resulted in the warning.

Automation can also augment the locomotive engineer's decision making. Software algorithms can assist the locomotive engineer to operate the train in the most economical and efficient ways while ensuring safety. As part of the ARES project, Burlington Northern $(\mathrm{BN})$ planned an energy management software module that would calculate train control movements using track profile, speed limits, motive power, consist profile and special conditions such as slow orders (Welty, 1988). Continuously determining the correct train control throttle and brake settings based upon these multiple criteria is beyond the locomotive engineer's cognitive ability. This automation assistance could reduce the amount of control adjustments by the cab crew. By providing more accurate information, the locomotive engineer could operate the train with fewer control actions. Alternatively, the train control automation could operate similarly to an autopilot in an airplane or cruise control in an automobile. In this mode, the locomotive engineer would monitor the actions of the automation.

The PTC system envisioned with ATCS and ARES will also reduce the number of displays in the locomotive cab. Many of the individual displays found in current generation locomotives such as the speed indicator and brake pressure displays, would be consolidated into a smaller group of one or two centralized displays (Watson, 1990). Instead of the conventional electromechanical displays, the information would be displayed on a CRT. Recently manufactured locomotives include these types of displays. The ATCS display specifications and the ARES displays share a common heritage. The same company, Rockwell International contributed to the development of specifications for ATCS and developed the displays for ARES. New locomotives based on the ARES displays continue to be built. Likewise, some new locomotives use a variant of the ATCS specification.

Figure 6-1 shows an example of such a display. This electronic display was developed as part of ARES. However, because the actual area in which this information is displayed is smaller than the amount of space taken by the electromechanical displays, not 
all the information can be displayed at once. Multiple screens are necessary to display all the information and a control mechanism is needed to access all the information. System designers developed multiple screens to handle this problem. In ARES, this was addressed by reserving one portion of the screen for vital information and allowing non-vital information to be displayed based on the engineer's requirements. Information could be displayed in textual or graphic format depending upon the engineer's requirements.

Some of these principles are discussed in Section 5.5 on dialog design. Integrated displays may be designed that combine different information elements that shows more information that the sum of its parts. The experimental display shows the in-cab signals, current speed and the brake profiles for full service and emergency braking.

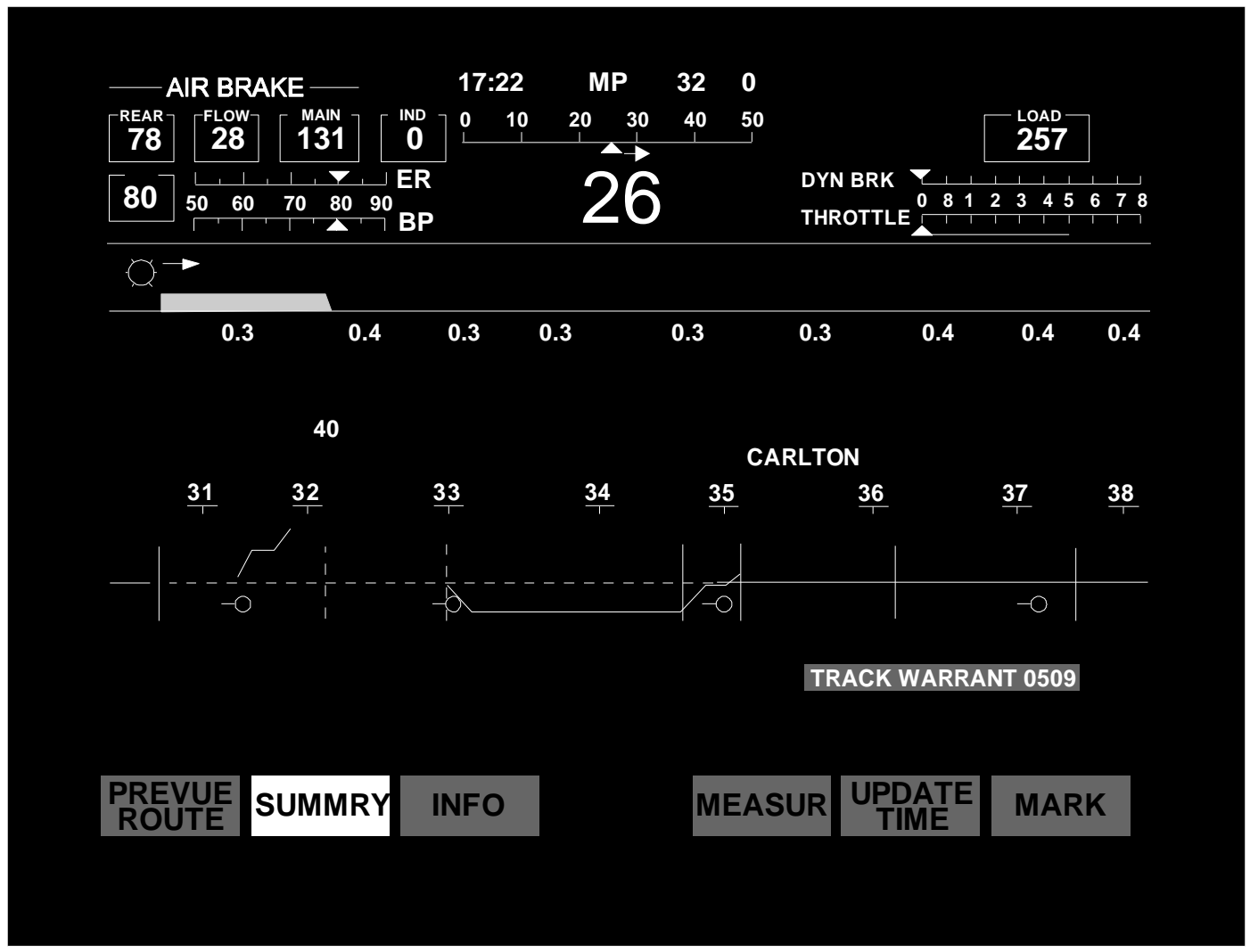

Figure 6-1. CRT Display. (From Train Situation Indicators, TEAM Technical Bulletin, Burlington Northern, 1991.)

\subsubsection{Human Factors Considerations}

How automation in the locomotive cab is implemented will have significant implications for the skill requirements and performance of the cab crew. Sheridan et al. (1994) identified 
two types of automation philosophies adopted by foreign railroad currently using automation for high speed rail operation. One philosophy, adopted by the French and Japanese railroads, uses automation to assist the passenger locomotive engineer in tactical decisions regarding train control. In this situation, the engineer is actively engaged in operating the train. A second philosophy, adopted by the German railroads, uses technology that allows the locomotive engineer to choose automation to control the train, while the locomotive engineer monitors the conditions of the train and uses the information provided by the automation to support strategic decisions required under unusual conditions.

Two concerns with automation in which the operator is a supervisor of the automation, is the level of situational awareness, and the ability of the operator to respond should the automation fail. In supervisory control, the locomotive engineer is not actively engaged in making decisions about train control. The engineer may miss a failure of the automation. Missing a failure may occur for two reasons. First, the engineer may not be sufficiently aware of what is happening around him or her, since the automation is making the train control "decisions." The engineer may no longer be alert to the environment around him (i.e., status of the locomotive, consist, and wayside). In an evaluation of a control automation system, Lanzilotta (In preparation) examined the effects of three automation modes: manual only, partial control automation and full control automation. Detection of automation failures were poorest in the partial automation mode. The results of this study suggest that users of automation may have difficulty in properly allocating their attention between events occurring outside the locomotive $\mathrm{cab}$ and events occurring inside the cab.

Second, the engineer may not adequately understand how the automation responds to various situations. The engineer needs to understand how the automation operates so that failure conditions can be detected. This means designing a system that is consistent with the "typical" locomotive engineer's mental model of how the automation works. Training should also be considered so that the engineer can acquire an accurate mental model of the system. The automation should also be designed to communicate with the humans in the system, about its own functioning.

In situations where the automation fails, the engineer may not be able to respond appropriately. With the automation regularly making train control decisions, the engineer may forget some of 
the knowledge required to respond. Also, the engineer's lack of situation awareness will increase the time before a response is made, while the engineer evaluates the situation.

Mode awareness is a related issue. In a system where there may be multiple levels of automation or modes, mode awareness is required because a different set of responses is required for each mode. For example, with a programmable function key interface, the engineer may use the same set of physical control inputs to access different portions of the system. In different automation modes, the same function key performs different actions. If it is not clear which mode the system is in, the engineer may be 'surprised' by the system response to his or her control input. Mode awareness problems tend to occur in complex systems where the automation is highly autonomous, has a high degree of authority, and communicates information poorly about its status (Sarter and Woods, 1995). The result is a mismatch between expected and actual behavior. The automation must be designed to communicate clearly what the automation is doing.

The question of what type of automation to provide is open to debate. Automation does not have to be an all or nothing proposition. In the aviation industry, Endsley and Kaber (In preparation) has proposed a model in which different levels of automation can be associated with different tasks. In flight operations, automation is available for every aspect of flight. During departure and arrival phases of flight, workload level is high. During the cruise phase, workload level tends to be low. However, as currently implemented, flight automation tends to increase workload during the high workload periods, while decreasing workload during the low workload periods (Billings, 1991). Regardless of how the automation is implemented, automation would be more helpful if it could smooth out the variations in workload level. In the train operations, system designers, will need to remain alert to the effects of automation on workload.

\subsection{COMMUNICATIONS}

\subsubsection{Operational Issues}

Currently, communications between the locomotive engineer and the dispatcher use two-way radio. Although voice communications by radio have improved over time (FRA, 1994), the channels that carry this information can be congested, 
misused, and suffer from hardware related problems. These problems decrease the efficiency of information transfer between dispatcher and train crew and increases the likelihood of miscommunication and the potential for an accident. Some dispatching districts report congestion devoted to safety related communications (FRA, 1994). Congestion of particular radio frequencies arises in part when channels intended for road train use are used by yard masters, terminal switching crews, and maintenance of way crews. In addition, train dispatchers and railroad employees in the field do not always comply with required radio standards and procedures.

Hardware problems manifest themselves in a number of ways. These problems include: "bleedover" between neighboring dispatcher districts, incompatible communications systems within dispatcher offices, and poorly functioning or disabled radios in the locomotive. The noisy acoustical environment in some locomotive cabs impairs the ability of train crews to monitor and respond to radio transmissions, which themselves may be noisy. These problems may be exacerbated when crew members have hearing losses.

Problems in transferring information between dispatcher and train crew also result from the problems inherent in voice communications. For example, radio communications take place over noisy channels that increase the difficulty in understanding the message. In response to this noise, air traffic controllers have adopted a standardized phraseology and procedures that dictate the order in which words are spoken and how to conduct the conversation.

Communications between dispatcher and train crew also include standardized phraseology and operating procedures. However, the standardized phraseology is minimal (CFR 49 Part 220) and can vary with operating rules. Even with standardized phraseology and operating procedures, communication errors occur that contribute to accidents. Some of these errors include:

message composition errors, errors in phraseology and delivery technique, and readback/hearback errors (Prinzo and Britton, 1993).

A form of digital communications called data link has the potential to solve many of these problems. In a data link system, messages are entered into a computer at one location and 
conveyed to a computer at another location. The receiver can then view these messages on an electronic display. Combining data link with voice communications has a number of advantages over conventional voice communications, alone. First, some of the messages can be diverted to data link, alleviating some of the congestion on the voice channels (Kerns, 1994). Second, data link messages can be displayed visually, reducing the demands on the train crews' memories. This information can be saved on the train's on-board computer and eliminates the requirement to write train orders. The visual display also reduces errors related to dispatchers' conveying train orders and errors associated with train crews' hearing and transcribing of these train orders (FRA, 1994).

In a simulation comparing voice communications with data link (Ditmeyer and Vanderhorst, 1992), data link reduced the number of transmissions required for each transaction compared with voice communications. In addition to reducing the overall amount of time needed for communication, it also reduced communication delays between dispatcher and train crew, reduced the number or errors compared to voice communications. While data link appears to make the communication process more efficient, it will not eliminate the need for traditional voice communications. Voice radio communications will remain important for handling special circumstances such as departures from normal operations, emergencies, and failures of the data link system.

\subsubsection{Human Factors Considerations}

More research as to the use of data link in a railroad environment is required to understand how to implement data link to maximize safety and efficiency. However, a data link type system could be critical to a proposed new PTC system. For a specific transaction, sender and receiver should not have to switch between voice and data link to complete a transaction (Kerns, 1994). Multimedia transactions are unnecessarily complex. Regardless of the communication medium, the communication process should be consistent. If voice

transmissions require an acknowledgment, so too should data link transmissions.

While data link offers many advantages for the train crew, there are some drawbacks, that will affect train crew performance and the types of errors that occur. Visual workload can increase with data link, while auditory workload decreases (Kerns, 1994). 
Information that was conveyed verbally will be displayed visually. The locomotive engineer will need to allocate his attention between tasks that have always required his visual attention and new tasks required by data link. The dispatcher and train crew will also have to keep track of two communications modes instead of one.

All aspects of information conveyed by speech are not communicated equally well, visually. For example, train crews can pick up cues about how busy the dispatcher is from listening to the speed and intonation of the dispatcher's voice that is not communicated visually, by simple text messages. Additionally, the party-line nature of radio communications means that the train crew may receive information about other events and assist in maintaining situation awareness. This information can be lost when data link transmissions are limited to those that directly affect the train crew. Just as speech communications are subject to errors (i.e., transposition of numbers), so too are visual communications. Instead of confusing numbers which sound alike, the train crew may confuse numbers that look alike (Kerns, 1994). One solution to this problem, is to add speech generation technology that so that the train crew can receive messages by either visual or auditory modes.

\subsubsection{Recommendations}

The following design guidelines were adapted from SAE recommendations for the use of data link in aviation (SAE Aerospace Recommended Practice 1994).

- Some means should be available for urgent communications for cases where data link communications become unavailable.

- As new data link services are developed and implemented, they should remain compatible with established procedures and promote predictable operator responses.

- Data link should compensate for the loss of party line communications found in voice environment. Information about risk level, controller workload, traffic density, request urgency and other aspects can be inferred from voice communications and should be available in the data link environment.

- The system should indicate what mode of communication is 
available and which capabilities are appropriate. In a multimedia communication system, communication procedures should be consistent. If acknowledgment is required by voice, it should also be required by data link.

- Data link should inform the operator of its current operational status. The system should notify the operator of incoming messages, maintain a queue of pending messages, display messages and maintain a log of messages that have been viewed, including operator response, where applicable.

- Messages should be delivered in the order in which they are sent within a given priority level.

- Data link delivery should facilitate error identification notification and recover within the time required for safe recovery. Data link should be designed to detect and trap potentially critical errors as early in the communication process (i.e., out of range values) as possible.

- Data link should have the capability for the operator to send messages with an emergency or distress signal that triggers a unique alert that immediate attention is required.

- Data link should identify the source and destination of transmitted messages unambiguously. The system must ensure that all messages are from a legitimate source. The time a message is sent should be available to help estimate the validity.

- Provide capability for the operator to transmit an "unable/reject" response to a movement authority. Operator should also be able to provide explanation by voice or data link.

- Explicit action should be required to approve transmission and to acknowledge messages.

- Data link should not prevent the operator from performing other high priority task by requiring excessive attentional resources or tying up equipment needed for other task with data link functions.

- Clearly distinguish between various human computer interface functions of the system. Indicate the type and format of data expected. Data link system should ensure that 
data intended for one purpose cannot be used erroneously for another.

- Data link should echo operator alphanumeric inputs within 0.2 seconds and respond to operator inputs with 0.5 seconds by either completing the processing or providing feedback that the input is being processed.

- The message urgency should be conveyed by a display code to facilitate operator recognition of urgency and appropriate response times.

- If not all pending messages not displayed, information shall be provided to notify the operator of the existence of a message queue.

- The system should clear status displays after a transaction is completed.

- New messages should not automatically cover and displace a message currently being displayed.

- The data link system should consider one or more of the following message generation capabilities: form filling text entry, free-form text entry, menu-selection of predefined messages, command entry of function key entry to quickly format messages.

- Data link enables information to be displayed graphically as well as textually. Graphic displays are ideal for displaying information about position in space and time. For instance where it was determined that a tactical display of train position, maintenance of way crews or other track equipment, this information can be displayed most effectively in a graphic format.

- Data link message content/phraseology/symbology should be designed to prevent misinterpretation and ease interpretation of the message. Message formats should use standard phraseology and unambiguous terms. Message formatting should allow the sender's message to be fully and accurately displayed. The operator should not have to enter the same information multiple times. 
(2) 


\section{REFERENCES}

American Society of Heating, Refrigeration and Air-Conditioning Engineers, Inc. Human Thermal Environments. ANSI/ASHRAE Standard 55-89 (55-92). Atlanta, GA: Author.

Askey, S. and Sheridan, T. (1996). Human Factors Phase II: Design and Evaluation of Decisions Aids for Control of High-Speed Trains: Experiments and Model. Washington, DC: Department of Transportation, Federal Railroad Administration.

Association of American Railroads (AAR). (1994). Railroad Facts. Washington, DC: Author.

Association of American Railroads (AAR). (1994a). Locomotive System Integration Architecture Specification M-591. Washington, DC: Author.

Association of American Railroads (AAR). (1994b). ATCS Specification 320 Locomotive Displays and Controls. Washington, DC: Author.

Aurelius, J.P. (1971). The Sound Environment in Locomotive Cabs. Report No. FRA-RP-71-2A. U.S. Department of Transportation, Federal Railroad Administration.

Austin, A. and Drummond, P.D. (1986). Work problems associated with suburban train driving. Applied Ergonomics. Vol. 17 (2), pp. 111-116.

Bailey, R.W. (1989). Human Performance Engineering (2nd ed.). Englewood Cliffs, NJ: Prentice Hall.

Bainbridge, L. (1987). Ironies of automation. In J. Rasmussen, K. Duncan, and J. Leplat (eds.) New Technology and Human Error. New York: John Wiley and Sons.

Ben-Yaacov, G. (1991). Improvement of ATCS operational safety and efficiency through human factors applications. Transportation Research Record. No. 1314. Washington, DC: Transportation Research Board, National Research Council.

Billings, C.E. (1991). Human-Centered Aircraft Automation: A Concept and Guidelines. NASA Technical Memorandum 103885. Moffet Field, CA: National Aeronautics and Space Administration.

Boff, K.R. and Lincoln, J.E. (Eds.) (1988). Engineering Data Compendium. Wright-Patterson Air Force Base, OH: Harry G. Armstrong Aerospace Medical Research Laboratory.

Branton, P. (1979). Investigations into the skills of train-driving. Ergonomics. Vol. 22 (2), pp. 155-164. 
Human Factors Guidelines for Locomotive Cabs

Bridger, R., (1988). Postural Adaptations to a Sloping Chair and Work Surface. Human Factors, Vol. 30, No. 2, pp. 237-247.

Brown, C.M. (1988). Human-computer interface guidelines. Norwood, NJ: Ablex.

Brown, D. (September 1994). The F40: an engineer's engine. Trains. p.74.

Buck, Leslie and Lamonde, Fernande. (1993). Critical incidents among locomotive engineers. Safety Science. Vol. 16 (1), pp. 1-18.

Burgess, J.H. (1986). Designing for Humans: The Human Factor in Engineering. Princeton, NJ: Petrocelli Books.

Burlington Northern. (1989). Headsets. TEAM Technical Bulletin. Overland Park, KS: Author, Vol. 3, (9).

Burlington Northern. (1991). Train Situation Indicators. TEAM Technical Bulletin. Overland Park, KS: Author, Vol. 5, (2).

Canadian National Railway. (1987). CNR locomotive cabs 1973 to 1986 Summary: Safety, comfort and ergonomic considerations. Montreal, Quebec: Author.

Cardosi, K. and Murphy, B. (1995). Human Factors in the Design and Evaluation of ATC Systems, U.S. Department of Transportation, Federal Aviation Administration.

CFR 49 Part 220. (199x). Radio Standards and Procedures. Code of Federal Regulations.

Chaffin, D.B. (1987). Biomechanical aspects of workplace design. In G. Salvendy (ed.) Handbook of Human Factors. New York: John Wiley and Sons.

Chase, M. (Dec. 12, 1994). To Help Avoid Blood Clots, Stretch Those Legs. Wall Street Journal.

Corey, G.M., Yoshino, R.T. and Lorenzen, M.J. (1989). Development of a console cab for six axle freight locomotives, RTD Volume 3 Rail Transportation, The Winter Annual Meeting book No H00578-1989. New York: The American Society of Mechanical Engineers.

Department of Defense. (1980). Military Handbook, Human Factors Engineering Design for Army Material. US Army Document MIL-HDBK-759A (MI).

Department of Defense. (1988). Human engineering design criteria for military systems, equipment and facilities. Military Standard 1472A.

Department of Defense. (1989a). Keyboard Arrangements (MIL-STD-1280). Washington, DC: Author. 
Department of Defense. (1989b). Human Engineering Design Criteria for Military Systems, Equipment and Facilities (MIL-STD-1472D).

Devoe, D.B. and Abernathy, C.N. (1977). Maintaining alertness in railroad locomotive crews . Report FRA/ORD-77/22. U.S. Department of Transportation, Federal Railroad Administration.

Dillon, A. (1994). Designing Usable Electronic Text. Bristol, PA: Taylor and Francis.

Ditmeyer, S. R. and Vanderhorst, J. (1992). Data Link Applications on a Railroad. Washington, DC: Association of American Railroads, $C \& S$ Division, Committee Reports and Technical Papers. P. 657-664.

Dumas, J. S. (1993). A Practical Guide to Usability Testing. Norwood, NJ: Ablex.

Endsley, M.R. and Kaber, D.B. (Unpublished manuscript). Level of Automation on Performance, Situation Awareness and Workload in a Dynamic Control Task.

Federal Railroad Administration. (1994). Railroad Communications and Train Control: Report to Congress. Washington, DC: U.S. Department of Transportation, Federal Railroad Administration.

Ford, R. (1978). Engineers look at drivers' cabs. Modern Railways. Vol. 35, pp. 22-37.

Galitz, W.O. (1993). User-Interface screen design. Wellesley Hills, MA.: QED Information Sciences.

Gamst, F.C. (1975). Human factors analysis of diesel electric locomotive cab. Human Factors, Vol. 17 (2), pp. 149-156.

Gilmore, W.E., Gertman, D.I., and Blackman, H.S. (1989). The user-computer interface in process control: A human factors engineering handbook. New York: Academic Press.

Grandjean, E. (1987). Design of VDT workstations. In G. Salvendy (Ed). (1987). Handbook of human factors. New York: John Wiley and Sons.

Grandjean, E. (1988). Fitting the task to the man (4th ed). New York: Taylor \& Francis.

Hale, A. and Jacobs, H.H. (1975). Proposed Qualification Requirements for Selected Railroad Jobs. Report No. FRA-ORD-75-44. U.S. Department of Transportation, Federal Railroad Administration.

Hartemann, Francois, Favre, Bernard. (1990). Human factors for display and control. Vehicle Electronics in the 90's: Proceedings of the International Congress on Transportation Electronics. IEEE cat N90CH2763-7, pp. 263-270. 
Human Factors Guidelines for Locomotive Cabs

Hedberg, G. (1987). Evaluation of the driver's cab in the R5 engine. Applied Ergonomics Vol. 18 (1), pp. 36-42.

Helander, M. (1988). Handbook of human-computer interaction. New York: Elsevier/North Holland.

Heron, R.M. (1988). Ergonomics evaluation of C.P. Rail's new road freight locomotive cab design. Montreal, Quebec: CP Rail System.

Hopkin, V.D. (1992). Issues in color application. In H. Widdel and D.L. Post (eds.) Color in Electronic Displays. New York: Plenum Press.

Human Factors Society. (1988). American National Standard for Human Factors Engineering of Visual Display Terminal Workstations. ANSI/HFS-100-1988. Santa Monica, CA: Author.

Hyyama, Y. (1980). Systems-ergonomics approaches to design and operation of today's railroads. Human Factors. Vol. 22(1), pp. 15-24.

Ivergard, T. (1989). Handbook of Control Room Design and Ergonomics. New York: Taylor and Francis.

Jankovich, J. (1972). Human Factors Survey of Locomotive Cabs. FRA-OPP-73-1. U.S. Department of Transportation, Federal Railroad Administration.

Karbowski, C.J., Ben-Yaacov, G., and Blass, D. (1991). Advances in flat panel display technology and applicability to ATCS on-board terminals. Transportation Research Record. No. 1314. Washington, DC: Transportation Research Board, National Research Council.

Kerns, K. (1994). Human Factors in ATC/Flight Deck Integration: Implications of Data Link Simulation Research. (MP-94W0000098) McLean, VA: MITRE.

Kerst, J.O. (1991). Ergonomic evaluation of locomotive cab seating for CP rail. Report No CMO278) Montreal, Quebec: CP RAIL Locomotive Development Project.

Keuhn, G. (1992). Advanced Train Control System Evaluation. Report No. FRA/ORD-92/32. U.S. Department of Transportation, Federal Railroad Administration.

Kingsley, E., Mason, S., Pethick, J. and Simpson, G.C. (1980). Ergonomic Principles of Underground Locomotive Design: Guidelines for Designers. Roxburgh Place Edinburgh Scotland: Institute of Occupational Medicine.

Lanzilotta, E. and Sheridan, T. (1996). Human Factors Phase III: Effects of Control Automation on Operator Performance. Washington, DC: Department of Transportation, Federal Railroad Administration. 
Laporte, F. and Jones, T. (1994). Ergonomic Design of Locomotive Cabs. Presentation made at the Southeast regional conference of the Association of American Railroads.

Lee, N.S., Schneider, L.W., and Ricci, L.L. (1990). Review of Selected Literature Related to Seating Discomfort, University of Michigan, Transportation Research Institute, UMTRI-9012.

Lueder, R. and Noro, K. (Eds.) (1994). Hard Facts about Soft Machines. Bristol, PA: Taylor and Francis.

Marcus, A. (1992). Graphic Design for electronic document and user interfaces . New York: ACM Press.

Mason, S. (1987). Proceedings of the Seminar on the Ergonomics of Locomotives and Free-Steered Vehicles. Report No. TM/87/05. Edinburgh, Scotland: Institute of Occupational Medicine.

Matias, E., MacKenzie, I.S., and Buxton, W. (1993). Half-QWERTY: A one-handed keyboard facilitating skill transfer from QWERTY. Proceedings of INTERCHI '93: Conference on Human Factors in Computing Systems. pp. 88-94. New York: Association for Computing Machinery (ACM).

Mayhew, D. (1992). Principles and guidelines in Software user interface design. Englewood Cliffs, NJ: Prentice-Hall.

McDonnell Douglas Corporation. (1972). Railroad Engineman Task and Skill Study. U.S. Department of Transportation, Federal Railroad Administration.

Michaut, G.M.E. and McGaughey, T.P. (1972). "Work conditions and equipment in diesel locomotives: feasibility study and recommendations." Report No. 72-11. Ontario, Canada: Canadian Institute of Guided Ground Transport, Queen's University, Kingston.

Miller, D.P. and Swain, A.D. (1988). Human error and human reliability. In M. Helander, (ed). Handbook of human-computer interaction. New York: Elsevier/North Holland.

National Aeronautics and Space Administration (NASA). (1989). Man-systems integration standard (NASA-STD-3000 Revision A) Houston, TX: NASA-Johnson Space Center.

National Space Technology Laboratories, Engineering Laboratory. (1982). Analysis of locomotive cabs. Final Report. Report No DOT/FRA/ORD-81/84). Washington, DC: U.S. Department of Transportation, Federal Railroad Administration.

Norman, K.L. (1991). The Psychology of Menu Selection: Designing Cognitive Control of the Human/Computer Interface. Norwood, NJ: Ablex. 
Human Factors Guidelines for Locomotive Cabs

Oborne D.J. and Clarke, M.J. (1974). The Determination of Equal Comfort Zones for Whole-body Vibration. Ergonomics, Vol. 17, No. 6, pp.769-782.

Parish, R.N., Gates, J.L., Munger, S.J., Grimma, P.R. and Smith, L.T. (1982). Development of design guidelines and criteria for user/operator transactions with battlefield automated system. (Phase II. Final report: Volume II. Prototype and Handbook for Material Developers . Alexandria, VA: U.S. Army Research Institute for the Behavioral Sciences.

Parker, C.W. (1974). Design and operation of remote-controlled locomotives in freight trains. Radio Engineers Journal.

Powell, A.J. and Cartwright, A. (1977). The design of drivers' cabs. Proceedings of the Institution of Mechanical Engineers. Vol. 191 (33), pp. 195-205.

Prinzo, O.V. and Britton, T.W. (1993). ATC/Pilot Voice Communications: A Survey of the Literature. Washington, DC: U.S. Department of Transportation, Federal Aviation Administration.

Progressive Railroading. (May 1994). High tech advances keep railroads rolling. Author. p.41.

R \& R Associates. (1979). Crewmembers evaluation of locomotive cab seat design characteristics : a survey. Report submitted to Association of American Railroads, Washington, DC.

Radford, R.W. The development of locomotive cab and body construction for Canadian National Railways. Unpublished manuscript. Available from R.W. Radford, Office of chief of M.P. \& C.E. Canadian National Railways, Montreal, Quebec.

Ramsey J.D., Burford, C.L., Beshir, M.Y., and Jensen, R.C. (1983). Effects of Workplace Thermal Conditions on Safe Work Behavior. Journal of Safety Research, Vol. 14 (3), pp. 105-114.

Ramsey, J. D. (1989). Assessment of warnings based on an ergonomic accident sequence model. International Journal of Industrial Ergonomics, Vol. 4 (3), pp. 195-199.

Riley, M.W., Stentz, T.L., Moore, B., McMullin, D., and Glismann, C. (1991). SD-60M Locomotive American wide cab ergonomics study. Final Report \& summary. Lincoln, NE: Burlington Northern Railroad.

Roach, D.E. and Rockwell, T.H. (1980). Locomotive cab seat evaluation: Final report (Report No R-404) Chicago, IL: Association of American Railroads Applied Technology Division.

Robinson, J. (1978). Locomotive Cab Design Development - Vol. IV Recommended Design, Report No. FRA/ORD-76-275.4. U.S. Department of Transportation, Federal Railroad Administration. 
Robinson, J., Piccione, D., and Larners, G. (1976). Locomotive Cab Design Development - Vol. 1 Analysis of Locomotive Cab Environment \& Development of Cab Design Alternatives, Report No. FRA/ORD-76-275.1. U.S. Department of Transportation, Federal Railroad Administration.

Rockwell, T.H. and Kiger, S.M. (1989). A Survey of Railroad Industry Perceptions Regarding Needed Locomotive Cab Design Improvements, DOT/FRA/ORD-89/03. U.S. Department of Transportation, Federal Railroad Administration.

Rogers, S.P. and Myers, L.D. (1993). Development of an intelligent system to aid in avionics display design. AIAA/IEEE Digital Avionics System Conference. New York: American Institute of Aeronautics and Astronautics.

SAE Aerospace Recommended Practice (1994). Human engineering recommendations for data link systems. (ARP-4791). Warrendale, PA: SAE International.

Salvendy, G. (Ed) (1987). Handbook of human factors. New York: John Wiley and Sons.

Sanders, M.S. and McCormick, E.J. (1994). Human Factors in engineering and design (7th ed) New York: Wiley \& Sons.

Sarter, N. and Woods, D.D. (1995). How in the world did we ever get into that mode? Mode awareness and supervisory control. Human Factors. 37 (1), 5-19.

Serber, H. (1990). New Developments in the Science of Seating. Human Factors Society Bulletin, 33 (2), pp. 1-3.

Sheridan, T., Lanzilotta, E., and Askey, S. (1994). Safety of High Speed Guided Ground Transportation Systems: Human Factors Phase I: Function Analysis and Theoretical Considerations. Washington, DC: Department of Transportation, Federal Railroad Administration.

Sheridan, T., Lanzilotta, E., and Yin, S. (1994). Safety of High Speed Guided Ground Transportation Systems Human Factors Phase 1: Function Analysis and Theoretical Considerations. U.S. Department of Transportation, Federal Railroad Administration.

Shneiderman, B. (1992). Designing the user interface: Strategies for effective human-computer interaction (2nd ed). Reading, MA: Addison-Wesley.

Smith, S. and Mosier, J. (1986). Guidelines for Designing User Interface Software. Bedford, MA: The MITRE Corporation, Electronic Systems Division.

Smith, S. (1987). Standards versus guidelines for designing user interface software. In M. Helander, (ed). Handbook of human-computer interaction. New York: Elsevier/North Holland. 
Human Factors Guidelines for Locomotive Cabs

Snyder, H. (1988). Image Quality. In M. Helander, (ed). Handbook of human-computer interaction. New York: Elsevier/North Holland.

Society of Automotive Engineers, Inc. (October 1987). Control Locations for Off Road Work Machines. SAE J898, Vol. 3, pp. 40.407-40.408. Warrendale, PA: Author.

Stanton, N. (1994). The Human Factors of Alarm Design. Bristol, PA: Taylor and Francis.

Templin, N. (Sept. 8, 1994). Vision Systems in Cars of Future May Ease Drivers' Fear of Dark. Wall Street Journal.

Tullis, T.S. (1988). Screen design. In M. Helander, (ed). Handbook of human-computer interaction. New York: Elsevier/North Holland.

Van Cott, H. P., and Kinkade, R.G. (Eds.) (1972). Human Engineering Guide to Equipment Design. Washington, DC: American Institutes for Research.

Vanderhorst, J. (1990). A Comparison of Voice and Data Link Communication in a Railroad Environment. Overland Park, Kansas: Burlington Northern Railroad Company.

Wainwright, D. (1969). BR's new seating science. Modern Railways. pp. 401-403.

Walraven, J. (1992). Color basics for the display designer. In H. Widdel and D.L. Post (eds.) Color in Electronic Displays. New York: Plenum Press.

Watson, R. (December 1990). Carriers, suppliers push 'clean cab'. Modern Railways. 35-37.

Welty, G. (September 1994). The high-tech explosion. Railway Age. pp. 55-58.

Welty, Gus, BN and ARES. (May 1988). "Control” in a new dimension. Railway Age. pp. 24-26.

White, J. (1988). Graphic design for the electronic age. New York: Watson-Guptill.

Wieringa, D., Moore, C., and Barnes, V. (1992). Procedure Writing Principles and Practices. Columbus, $\mathrm{OH}$ : Battelle Press.

Wilde, G.J. and Stinson J.F. (1980). Human Factors Considerations in Locomotive Cab Design . Ontario: Canadian Institute of Guided Ground Transport, Queen's Institute of Guided Ground Transport, Queen's University at Kingston, Report No. 80-9.

Wilde, G.J.S. and Stinson, J.F. (1980). Injuries in locomotive cabs. Journal of Safety Research Vol. 12 (4), pp. 179-184.

Williges, R.C., Williges, B.H., and Elkerton, J. (1987). Software interface design. In G. Salvendy (ed.) Handbook of Human Factors. New York: John Wiley and Sons. 
References

Woodson, W.E. Tillman, B, Tillman, P. (1992). Human Factors Design Handbook. (2nd ed) New York: McGraw. 


\section{Index}

\section{A}

AAR control stand, 66-67

abbreviations, 18, 27, 30, 33, 78, 141, 149

Advance Train Control System (ATCS), 116

air conditioning (see also ventilation), 12, 42

humidity, 39

shades, 12, 46

temperature, 12,43

windows, 12,45

alarms, 26, 112-115

design of, 26, 127

auditory, 27, 112, 127

false, 27, 128

alerter (see vigilance monitoring), 164

analog displays, 148

anthropometric requirements, 99

work-space envelope, 17

ARES, 4, 154, 171-173

ATCS, 3-4, 116, 120, 124, 129-130, 151, 154, 171-172

auditory displays (see also alarms), 23, 127 frequency range, 128

locomotive operating systems, 127

vigilance system, 112

when to use (should be used), 127

automation, 28-29, 129-136, 171

control task guidelines, 28, 135

human-centered automation, 28, 134

information management guidelines,

28,135

management by consent, 28, 134

management by exception, 28, 134

situational awareness, 29, 130, 132,

151

workload, 29, 130, 132

\section{B}

backward compatibility, 65-66

bi-directional operations, 67, 97 blink rate (see also flash coding), 31, 143

breadth vs depth trade-off, 121

brightness (see luminance coding), 139

C

cab layout, 15, 65, 71, 96

auxiliary items, 15, 77

clearances, 15, 68

controls and displays, 16, 67, 72

designing for an adjustable range, 16, 71

designing for extreme individuals, 16 , 71

designing for the average individual,

16,71

floor space, 15, 69

height of cab ceiling, 15, 68

maintenance and cleaning, 18, 19, 67

reach envelopes, 15, 75

remote operations, 15,74

visibility, 16, 71

work-space envelope, 17, 74

coding schemes, 25, 123, 153

color, 25, 73, 144

flash (see also blink rate), 31, 143

luminance (brightness), 29, 139, 143

reverse video, 32,143

size, 144

color, 25, 29, 32, 73, 110, 124, 144

command language, 117-118

computer displays and controls, 98

computer generated displays (see also

electronic displays), 27, 136

graphic information, 33, 148

hardware, 29, 117, 138

information coding, 31, 143

information placement, 30, 141

software, 30, 139

symbol design, 34, 149

typography, 32, 145 
computer input devices, 34, 150

keyboards, 25, 35, 36, 152

touchscreens, 36

computer technology, 1, 6, 177

consistency in key assignment, 125

control-display compatibility, 73, 98, 155

controls, 4, 8, 16-17, 22-23, 29, 98, 101-106, 167

movement, 105

placement, 31, 104, 109

spacing, 25, 105, 109

cooling (see air conditioning; ventilation), 12

crew comfort, $3,37,45,48,51$

\section{D}

data link, 177-180

design guidelines, 15, 22, 122, 137, 150

design principles, 24, 96

consistency, 22, 124

feedback, 25, 124

operator error, 26, 125

redundancy, 124

systems approach, 124

user-centered design, 98

design rules, 7,8

design standard, 5, 15

desktop workstation (see also workstation

design), 96

dialog design, 24, 115

alarm design, 26, 127

error management, 26, 126

error messages, 129

feedback and user guidance, 27, 128

function key menus, 24

memory load, 117

menus, 24, 118

principles, 24

recovery from errors, 117

digital displays, 33, 150

direct manipulation, 118-119

display clutter, 24, 153
E

electromechanical displays, 22, 106

arrangement, 111

glare elimination, 111

reliability of, 107

scale marking of, 109

size of dials, 108

electronic displays (see also computer

generated displays), 29, 136

abbreviations, 141

dark adaptation, 140

flicker, 138

glare, 30, 139

luminance and contrast, 138

maintenance, 139

resolution, 138

tabular formats, 141

wording, 141

error management, 26, 126

alarm design, 127

error messages, 27, 129

feedback, 128

prevent, 126

recovery from, 126

user guidance, 129

error tolerant design, 129

\section{F}

fatigue, 43

flash coding, 143

form fill-in, 118

function key menu-based interface, 153

function keys, 24-25, 153

abbreviation, 149

destructive effect, 25

feedback, 24, 124

label, 25, 123

placement, 25, 153

response time, 26, 125

soft, $25,120,153$

spatial relationship, 25, 123 


\section{G}

glare, 16, 30, 111, 139

graphic information, 32, 149

analog versus digital displays, 33-34,

148

symbol design, 34, 149

H

health problems, 39, 92

heating, 37-41

eveness, 39-40

glazing, 39

humidity, 39, 41

temperature, 40

highlighting (see also coding schemes), 143

color, 144

flash coding (see also blink rate), 144

luminance coding (brightness), 139

reverse video, 144

size coding, 144

humidity, 9, 10, 39, 41, 152

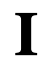

Information coding, 31, 143

color, 32

flash (see also blink rate), 31, 143

luminance, 32, 143

reverse video, 31,143

size, 33,144

information placement, 30, 141

grouping, 142

visual scanning, 31, 141

information technology, 3-6

ingress and egress, 20, 80

design, 20, 80

doors, $80-81$

emergency exit, 20, 81

latches, 20, 80-81

interaction style (see dialog design), 118
K

keyboard, 25, 35, 152-153, 155-156

arrangement, labeling and feedback, 155

function keys, 153

height, thickness and slope, 155

location, 156

$\mathbf{L}$

labels, 17, 77, 123

layout (see cab layout), 15, 71

locomotive design, 57,68

M

maintenance, 17, 43, 47, 52, 67, 76, 140

access, 18,78

inspection and testing, 79

labeling, 17, 78

repair, removal and replacement, 18, 78

menu systems, 118, 121-122

menus, 24

selection of, 24, 118

soft function key based, 25, 120

movement compatibility, 73-74

$\mathbf{N}$

navigation, 125,154

noise, $3,12,42,46$

active cancellation of, 50

brake venting, 50

exposure limits, 48

hearing loss, 48

horn location, 49

insulation from, 50

intelligibility, 48

isolated cab, 50

verbal communication, 48 


\section{$\mathbf{P}$}

Positive Train Control (PTC), 4, 129, 170

Positive Train Separation (PTS), 4, 129

Q

qualifier keys, 124, 154

$\mathbf{R}$

refresh rate, 29

response time, 26, 73, 21-122, 125

reverse video, 32, 144

$\mathbf{S}$

seat design, 21, 87-88, 90, 99

armrest height, 90

armrest width, 90

armrest length, 90

armrest padding, 90

back width, 90

back height, 90

covering, 21

cushion thickness, 90

features and adjustment, 90

fore-aft adjustment, 91

height, 91

pan slope, 90

pan size, 90

pan width, 90

seat back tilt, 91

swivel, 91

seating, 1, 3, 17, 21, 22, 44, 58, 85, 87, 97-99

adjustment, 92

alternative designs, 89

posture, 21, 59

vibration, 57

sentence structure, 33

situation awareness, 130, 132

soft function key menu, 120 spatial layout, 122

steps, 9, 125, 167

symbol shape, 34, 150

$\mathbf{T}$

toilet facilities, 13,51

cleaning, 55-56

lighting, 51

odor reduction of, $51,53,55$

odor produced by, 51, 53

size of, 53, 55

temperature, 54

ventilation, 51

touchscreens, 36, 157

device suitability, 157

feedback, 158

input strategy, 158

parallax, 157

physical characteristics and layout, 157

trackballs, 158

dimensions, resistance and clearance, 158

dynamic characteristics, 160

limb support, 158

typography, 32, 145

character height, 32, 145

sentence structure, 147

typeface, 145

white space, 33, 147

wording, 147

$\mathbf{U}$

usability, 5, 7, 120, 131, 154

user acceptance, 97

user-centered design, 9, 98

V

ventilation (see also air conditioning), 12, 38, 41

air quality, 12, 42

air speed, 12, 41 
cab pressure, 12, 42

drafts, 12, 42

effect of windows on, 12, 41

noise, 12,42

vibration, 14-15, 47, 57, 152

active control of, 63

comfort, effects on, 60-61

frequency range, 58

health effects of, 14, 58-60

isolation of, 62

measurement of, 61

vigilance monitoring, 112, 164

visibility, 14, 20, 71, 82

bi-directional travel, 97

effect of glare on, 32, 136, 139

of workstation layout, 71

long hood forward, 82

vision supplements, 20, 84

windows, 20, 84

voice communications, 160

\section{W}

warning signals (see also alarms), 114

white space, 30, 33, 141

wording, 18, 78, 148

working conditions, 2, 3, 8, 97-98

workstation design (see also electromechanical

displays, electronic displays, work-space

envelope), 17, 95, 96

arrangement of controls, 17, 23, 104

compatibility between controls and

displays, 73, 98

operation of controls, 102 